\title{
Análise e comparação de alguns métodos alternativos de seleção de variáveis preditoras no modelo de regressão linear
}

Matheus Augustus Pumputis Marques

\author{
DisSERTAÇÃO APRESENTADA \\ AO \\ Instituto De Matemática e Estatística \\ DA \\ Universidade De São Paulo \\ PARA \\ OBTENÇÃO DO TÍTULO \\ $\mathrm{DE}$ \\ Mestre em Estatística \\ Programa: Estatística \\ Orientador: Profa. Dra. Silvia Nagib Elian
}

Durante o desenvolvimento deste trabalho o autor recebeu auxílio financeiro do CNPq

São Paulo, Agosto de 2018 


\title{
Análise e comparação de alguns métodos alternativos de seleção de variáveis preditoras no modelo de regressão linear
}

\author{
Esta versão da dissertação contém as correções e alterações sugeridas \\ pela Comissão Julgadora durante a defesa da versão original do trabalho, \\ realizada em 04/06/2018. Uma cópia da versão original está disponível no \\ Instituto de Matemática e Estatística da Universidade de São Paulo.
}

Comissão Julgadora:

- Prof ${ }^{\mathrm{a}}$. Dr ${ }^{\mathrm{a}}$. Silvia Nagib Elian (orientadora) - IME-USP

- Prof. Dr. João Ricardo Sato - UFABC

- Prof. Dr. Rinaldo Artes - INSPER 


\section{Agradecimentos}

Ingressar em uma nova área para o mestrado foi um enorme desafio, e o apoio de minha família foi fundamental.

Agradeço a meus pais e minha avó pelo apoio constante.

Agradeço ao IME-USP, por ter me dado esta oportunidade.

Agradeço à Professora Silvia Nagib Elian, que foi minha orientadora em muito mais do que esta dissertação.

Agradeço ao Professor Luís Gustavo Esteves pela disponibilidade em me auxiliar em diversos momentos do Programa.

Agradeço também a todos os funcionários do IME que sempre foram muito solícitos e atenciosos a minhas perguntas e requerimentos, em especial a Regiane da Secretaria de Pós Graduação.

E claro, a todos os meus colegas do Mestrado, que me ajudaram muito a me adaptar às demandas da área da Estatística, e da Matemática como um todo.

E acima de tudo a Deus, pela força, persistência, diligência e paciência necessárias para completar esta jornada. 



\section{Resumo}

PUMPUTIS MARQUES, M. A. Análise e comparação de alguns métodos alternativos de seleção de variáveis preditoras no modelo de regressão linear. 2018. 78 f. Dissertação (Mestrado) - Instituto de Matemática e Estatística, Universidade de São Paulo, São Paulo, 2018.

Neste trabalho estudam-se alguns novos métodos de seleção de variáveis no contexto da regressão linear que surgiram nos últimos 15 anos, especificamente o - Least Angle Regression, o NAMS, Noise Addition Model Selection, a Razão de Falsa Seleção, RFS (FSR em inglês), o LASSO Bayesiano e o Spike-and-Slab LASSO. A metodologia foi a análise e comparação dos métodos estudados e aplicações. Após esse estudo, realizam-se aplicações em bases de dados reais e um estudo de simulação, em que todos os métodos se mostraram promissores, com os métodos Bayesianos apresentando os melhores resultados.

Palavras-chave: modelos lineares, regressão linear, seleção de modelos, seleção de variáveis, LARS, NAMS, FSR, RFS, LASSO Bayesiano, Spike-and-Slab LASSO. 



\section{Abstract}

PUMPUTIS MARQUES, M. A. Análise e comparação de alguns métodos alternativos de seleção de variáveis preditoras no modelo de regressão linear. 2010.78 f. Dissertação (Mestrado) - Instituto de Matemática e Estatística, Universidade de São Paulo, São Paulo, 2018.

In this work, some new variable selection methods that have appeared in the last 15 years in the context of linear regression are studied, specifically the LARS, Least Angle Regression, the NAMS, Noise Addition Model Selection, the False Selection Rate, FSR, the Bayesian LASSO and the Spike-and-Slab LASSO. The methodology was the analisys and comparisson of the studied methods. After this study, applications to real data bases are made, as well as a simulation study, in which all mehtods are shown to be promissing, with the Bayesian methods showing the best results.

Keywords: Linear Models, Linear Regression, model selection, variable selection, LARS, NAMS, FSR, Bayesian LASSO, Spike-and-Slab LASSO. 


\section{Sumário}

Sumário . . . . . . . . . . . . . . . . . . . . . viii

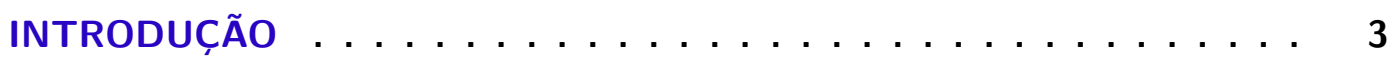

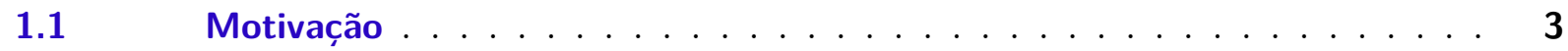

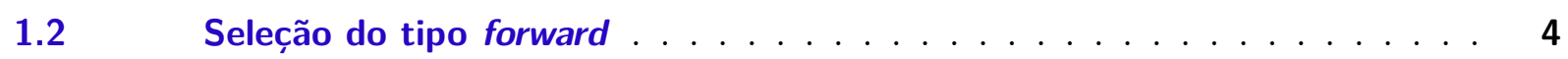

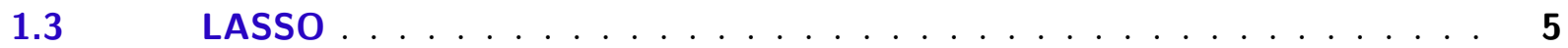

2 MÉTODO DE REGRESSÃO DOS MÍNIMOS ÂNGULOS - LARS . . . . 7

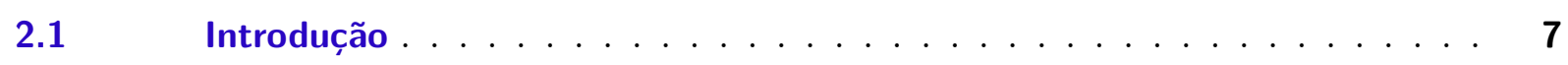

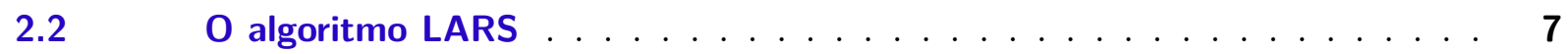

2.3 Comparação entre o método LARS e o método dos mínimos quadrados ordinários . . . . . . . . . . . . . . . . . . . 13

3 USO DE PSEUDOVARIÁVEIS NA SELEÇÃO DE VARIÁVEIS NOS MODELOS DE REGRESSÃO. . . . . . . . . . . . . . . . . . . . . . 17

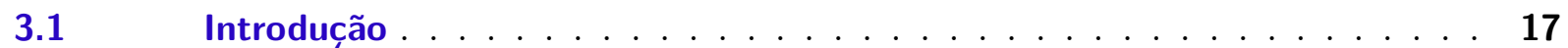

3.2 Método de seleção de modelos com a adição de ruído - NAMS . . . . . . . 17

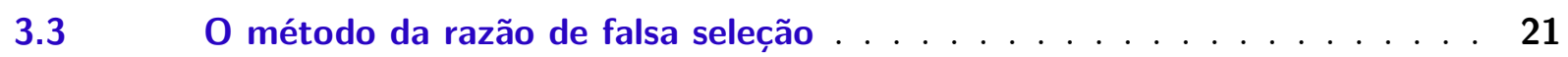

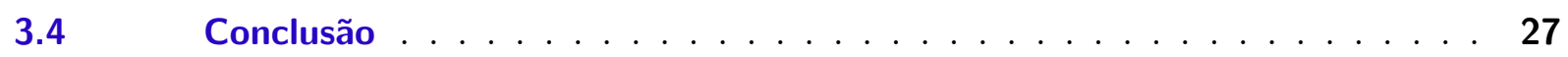

4 OS MÉTODOS DE LASSO BAYESIANOS PARA SELEÇÃO DE VARIÁVEIS 29

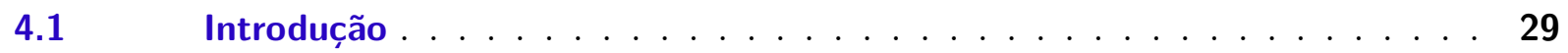

4.2 Seleção de variáveis via Modelos Hierárquicos e o procedimento Bayes empírico . . . . . . . . . . . . . . . . . . . 29

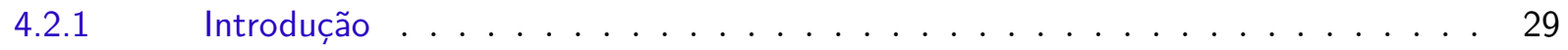

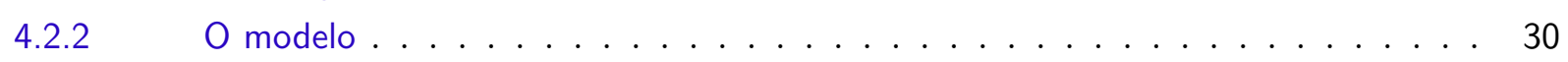

4.2.3 O uso do método de Bayes empírico para a estimação do LASSO . . . . . . . . . 33

4.3 Interpretação Hierárquica alternativa e Amostrador de Gibbs . . . . . . . . 34

4.3.1 Distribuição de Laplace como mistura de distribuições Normais . . . . . . . . . . 35

4.3.2 Uso do amostrador de Gibbs . . . . . . . . . . . . . . . . . . . 36

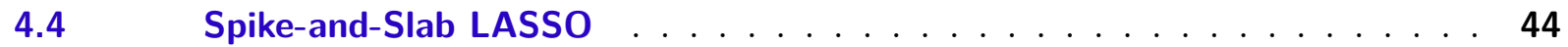

$4.5 \quad$ Aplicação de métodos bayesianos em genética . . . . . . . . . . . . . 49

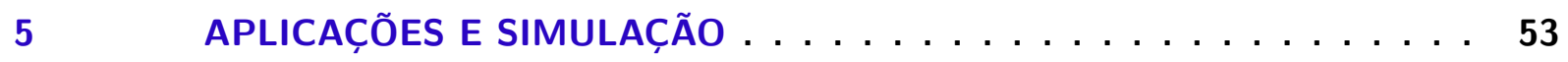

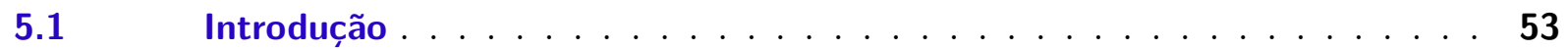

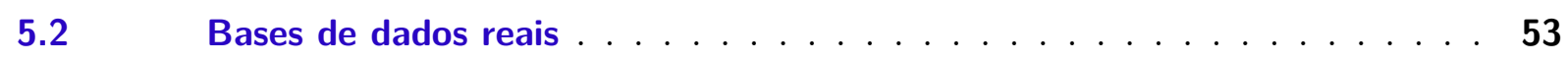

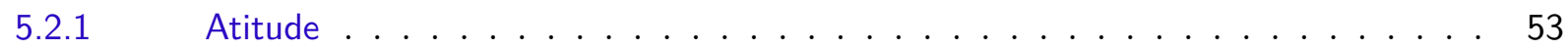

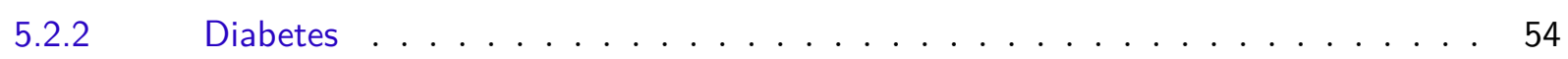

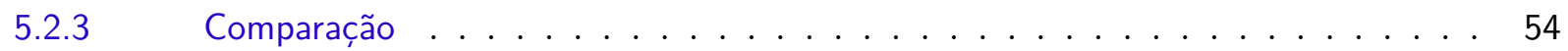

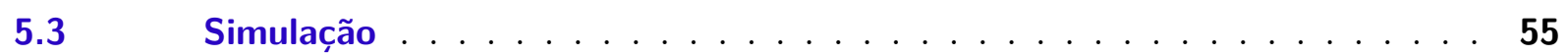


5.3.1 Cinco coeficientes das variáveis explicativas não nulos . . . . . . . . . . 55

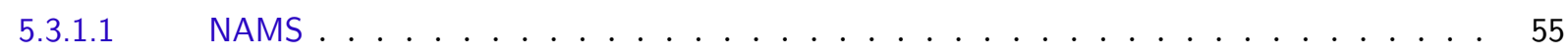

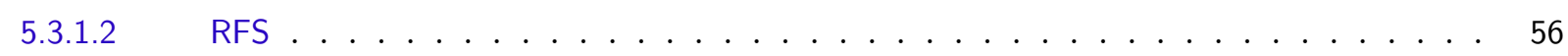

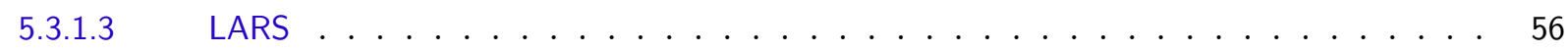

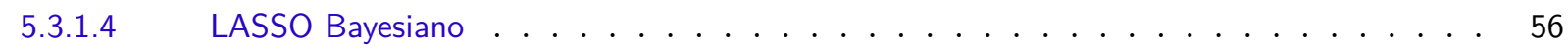

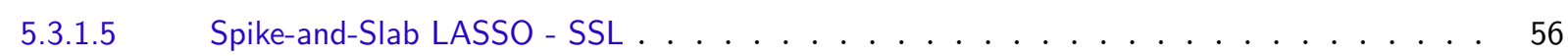

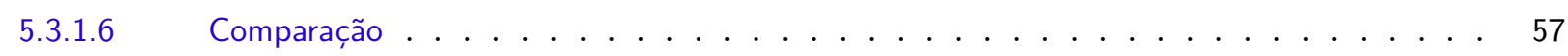

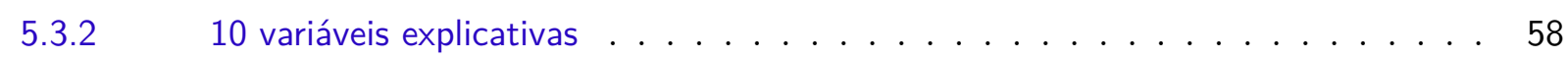

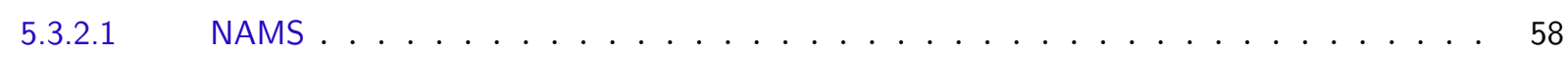

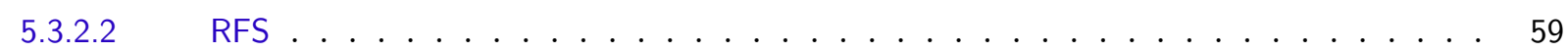

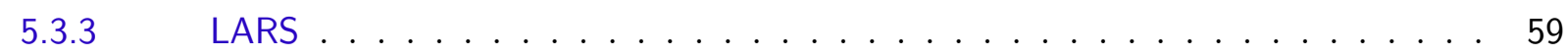

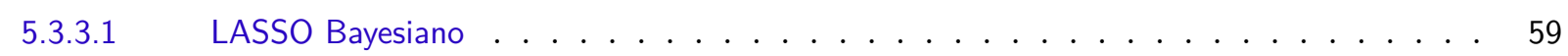

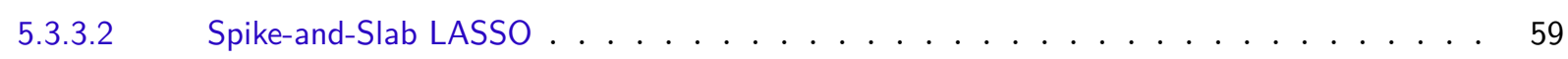

$5.3 .3 .3 \quad$ Comparação . . . . . . . . . . . . . . . . . . . . . 59

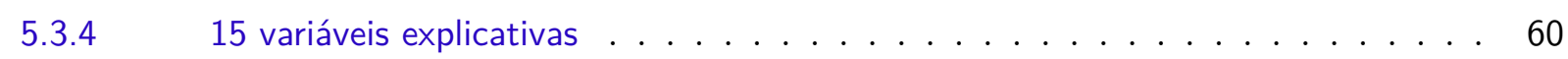

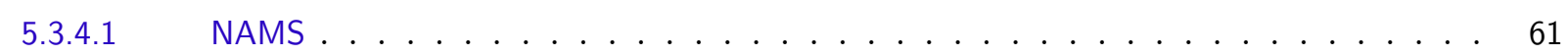

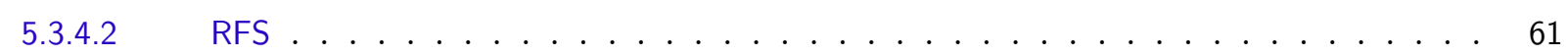

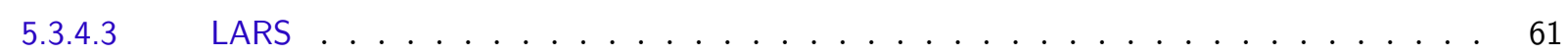

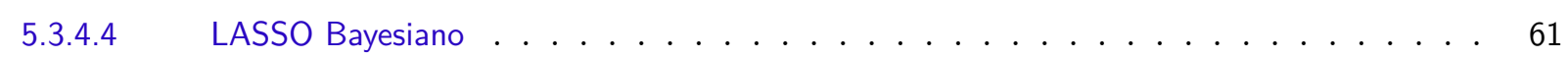

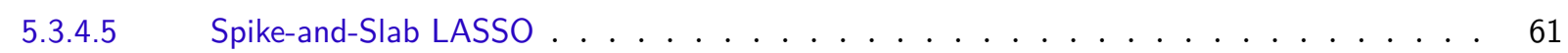

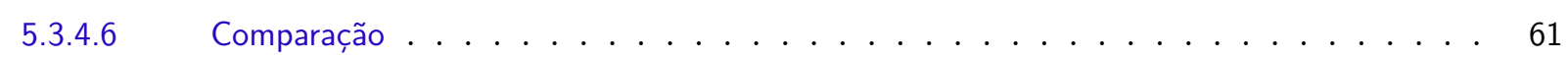

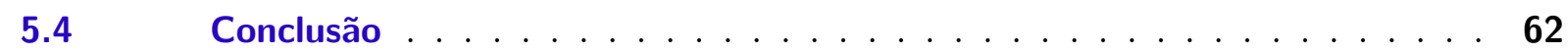

$.1 \quad$ Apêndice $A \ldots \ldots \ldots \ldots \ldots$

$.2 \quad$ Apêndice B - funções para a RFS e ajustes para o NAMS . . . . . . . . . 65

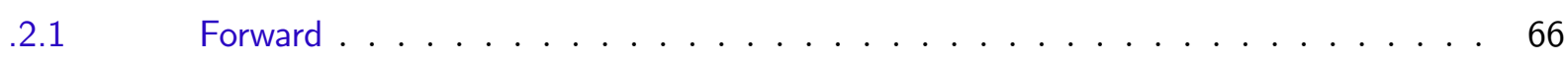

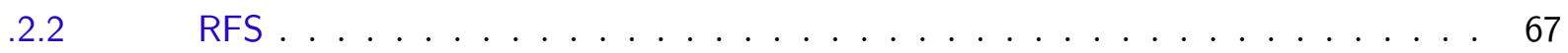

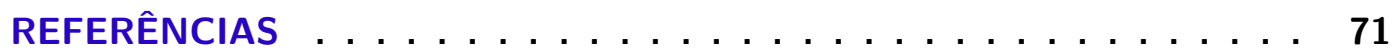





\section{Introdução}

\subsection{Motivação}

Um dos problemas mais importantes na área de pesquisa dos modelos lineares é a seleção de variáveis explicativas. Muitas vezes possui-se um banco de dados com um número muito grande de variáveis e deseja-se construir um modelo para explicar o comportamento de uma delas em função das outras. Incluir todas as variáveis no modelo traz dois problemas importantes. Em primeiro lugar, um número maior de variáveis explicativas que observações impossibilita o uso da regressão linear, devendo o pesquisador buscar outros métodos de estimação. Em segundo lugar, se o objetivo do trabalho é explicar a relação entre as variáveis, ou isolar os fatores mais relevantes para comportamento da variável em estudo, algo muito importante nos campos de Economia e Medicina, por exemplo, é natural que se deseje modelos enxutos, com o menor número possível de variáveis explicativas, mas com grande capacidade explicativa e preditiva.

Com esse objetivo, muitas técnicas foram desenvolvidas para a seleção de variáveis. As mais comuns, encontradas na maioria dos livros de regressão, são o forward, o backward e o stepwise, métodos com base em testes de hipótese.

Atualmente metodologias com base na Teoria da Informação, como o critério de informação de Akaike (AIC) e o critério de informação Bayesiano (BIC), também são extremamente populares, assim como o LASSO, um método que unifica seleção e estimação, mas ao fazer isso se afasta da metodologia de mínimos quadrados. Técnicas menos comuns incluem métodos com base em todas as regressões possíveis, como o método $C_{p}$ de Mallow's por exemplo (MALLOWS, 1973; MALLOWS, 1995).

O presente trabalho teve como objetivo fazer uma busca na literatura dos últimos 15 anos e selecionar algumas metodologias inovadoras que surgiram nesse período. Não se buscou fazer uma descrição exaustiva de todos os trabalhos sobre o tema escritos no período, apenas destacar algumas abordagens distintas entre si com potencial para se tornarem novas áreas de pesquisa. Um ponto inicial de busca foram as sugestões do livro de Ryan (2008, pp. 277-278) na área de novos métodos de seleção de variáveis.

Os métodos a serem discutidos neste trabalho são, em ordem, LARS, (EFRON et al., 2004), NAMS, (LUO; STEFANSKI; BOOS, 2006), RFS (FSR em inglês) (LUO; STEFANSKI; BOOS, 2006), LASSO Bayesiano (YUAN; LIN, 2005), (PARK; CASELLA, 2008a) e o Spike-and-Slab LASSO (ROČKOVÁ; GEORGE, 2016a).

A divisão em capítulos é feita da seguinte maneira: Este é o primeiro capítulo, que conta com, além dessa introdução, um resumo dos métodos forward e LASSO.

O Capítulo 2 trata do LARS (EFRON et al., 2004), que é uma extensão e generalização do LASSO, que também é menos custosa computacionalmente e possui uma interpretação geométrica por meio de projeções. Uma das vantagens desse método é que é possível obter com ele todas as 
soluções do LASSO, mas em um processo computacional com um número de etapas igual ao número de preditores (muito menos custoso que os métodos usuais de validação cruzada para o LASSO). A seleção de modelos é feita por meio de uma estatística $C_{P}$ modificada.

O Capítulo 3 trata de dois métodos que utilizam a inclusão de pseudovariáveis, o método de seleção de variáveis com a adição de ruído (LUO; STEFANSKI; BOOS, 2006) e o método da razão de falsa seleção (WU; BOOS; STEFANSKI, 2007).

O primeiro desses métodos é baseado na inclusão de ruído à variável resposta para estimar o viés do estimador do EQM e com isso ter uma indiciação de se o modelo está incluindo variáveis pouco importantes ou omitindo variáveis importantes, e com isso, calibrar o parâmetro de entrada em um método de seleção forward.

O segundo baseia-se na inclusão de pseudovariáveis na matriz de planejamento de modo a observar, em média, qual a proporção de variáveis pouco importantes em relação a variáveis importantes em modelos modelos escolhidos por métodos forward com valores diferentes para o parâmetro $\alpha$.

O Capítulo 4 trata de métodos Bayesianos.

Os métodos de Yuan, Joseph e Lin (2007) e Park e Casella (2008a) apresentam duas alternativas distintas para o LASSO Bayesiano utilizando modelos hierárquicos. Yuan, Joseph e Lin (2007) atribuem uma distribuição à priori para cada modelo possível e seleciona o modelo que apresentar a maior probabilidade à posteriori, enquanto Park e Casella (2008a), o mais utilizado atualmente, interpretam o estimador do procedimento LASSO como a moda de uma distribuição de probabilidades à posteriori, e estima o valor dos parâmetros do modelo com o auxílio do Amostrador de Gibbs.

O terceiro método é o Spike-and-Slab LASSO (ROČKOVÁ; GEORGE, 2016b), uma variação do LASSO bayesiano no qual a distribuição à priori do vetor $\boldsymbol{\beta}$ segue uma distribuição Spike-and-Slab, ou seja, uma mistura de distribuições de Laplace. O uso das prioris Spikeand-Slab possibilita duas vantagens importantes. Em primeiro lugar, elas permitem um termo de penalização que se adapta aos dados, o que é especialmente importante em situações que lidam com modelos esparsos, ou seja, que possuem inicialmente um grande número de variáveis explicativas porém a maior parte deles tem coeficiente zero. A segunda vantagem é que elas permitem a formulação de um algoritmo de seleção no qual o parâmetro de uma das duas distribuições Laplace (especificamente a "Spike") é sucessivamente alterado, de maneira a se tornar mais rígido a cada etapa.

O Capítulo 5 trata de aplicações, um estudo de simulação e comparações entre alguns dos métodos estudados nessa dissertação.

\subsection{Seleção do tipo forward}

O método de seleção do tipo forward é um dos mais simples. Como ele é a base para os dois métodos baseados em pseudovariáveis discutidos no Capítulo 3, tal método será explicado aqui, com base no livro de Ryan (2008, p. 269 - 271). 
Suponha que um modelo possui uma variável resposta $\boldsymbol{Y}$, com $\boldsymbol{Y}=\left(Y_{1}, Y_{2}, \ldots, Y_{n}\right)$ e uma matriz de planejamento $\boldsymbol{X}$ com $p$ variáveis, composta de vetores linha $\boldsymbol{X}_{1}, \boldsymbol{X}_{2}, \ldots, \boldsymbol{X}_{p}$, cada um deles $\boldsymbol{X}_{\boldsymbol{i}}=\left(X_{i 1}, X_{i 2}, \ldots, X_{i n}\right)$, que representam os valores de cada variável resposta relacionados ao valores do vetor $\boldsymbol{Y}$, e deseja-se selecionar algumas delas para criar um modelo estatístico. Inicia-se com 0 variáveis e define-se um nível de significância $\alpha$, que no procedimento forward também pode ser chamado de nivel de entrada. A seguir, ordena-se as variáveis explicativas pelo critério $\left|r_{Y X_{i}}\right|$ ou seja, o módulo do coeficiente de correlação entre $Y$ e $X_{i}$. Escolhe-se para entrar no modelo a variável dada por $\max _{i}\left|r_{Y X_{i}}\right|$. Para melhor notação, denota-se essa variável escolhida por $X_{e}$. Efetua-se então uma regressão linear de $\boldsymbol{Y}$ em $\boldsymbol{X}_{e}$. Caso o valor da estatística $t$ dessa variável indique sua não rejeição a um nível de significância $\alpha$, esta variável será permanentemente inclusa no modelo. Calcula-se então o coeficiente de correlação parcial entre $\boldsymbol{Y}$ e todas as demais variáveis não incluídas eliminado o efeito de $X_{e}$. A variável explicativa que apresentar o maior coeficiente de correlação parcial será incluída no modelo se sua contribuição foi estatisticamente significante. O procedimento continua para as outras variáveis até ou todas as variáveis estarem inclusas no modelo, ou uma variável for rejeitada no teste de significância. Nesse caso, ela não entra no modelo e o procedimento é encerrado.

O procedimento forward têm duas críticas principais. A primeira é que ele só leva em consideração no máximo $p$ modelos possíveis, sendo que existem sempre $2^{k}-1$ submodelos no total. Essa diferença pode ser significativa no caso de modelos com muitas variáveis explicativas. Em segundo lugar, o procedimento não leva em conta que a inclusão de novas variáveis pode tornar variáveis inclusas anteriormente não significantes, nem leva em conta o caso em que um grupo de variáveis são não significantes individualmente mas podem ser significantes conjuntamente.

Os métodos baseados em pseudovariáveis estudados no Capítulo 3 buscam selecionar o valor "ideal" para $\alpha$ a partir de muitas simulações.

\subsection{LASSO}

O LASSO é uma metodologia proposta por Tibshirani (1996). "LASSO" é um acrônimo, que significa Least Absolute Shrinkage and Selection, ou "menor encolhimento absoluto e seleção [de variáveis]". Seu nome também é uma referência a um método anterior conhecido como "Garrote" no qual ele foi inspirado.

Suponha-se um modelo linear nos mesmos termos do modelo anterior proposto para o forward, os estimadores dos parâmetros do LASSO são dados pela resolução do seguinte problema:

$$
(\hat{\alpha}, \hat{\boldsymbol{\beta}})=\operatorname{argmin}\left\{\sum_{i=1}^{n}\left(Y_{i}-\alpha-\sum_{j} \beta_{j} X_{i}\right)^{2}\right\} \quad \text { sujeito a } \sum_{j}\left|\beta_{j}\right| \leq t,
$$

no qual $\beta$ é o vetor de coeficientes de regressão associado às variáveis e $\alpha$ é o intercepto. Ou seja, é um método de mínimos quadrados restrito. O parâmetro $t \geq 0$ controla a "redução"do modelo. Essa redução refere-se ao número de preditores, já que, dependendo da escolha de $t$ alguns coeficientes de $\hat{\boldsymbol{\beta}}$ podem ser zerados. Isso equivale a retirar do modelo a variável ao qual aquele coeficiente era relacionado. A escolha do parâmetro $t$ é de extrema importância, 
ele determina se nenhum, alguns ou todos os coeficientes serão zerados. O autor propõe que se utilize de métodos de validação cruzada para escolher o parâmetro $t$, especificamente a validação cruzada generalizada e a validação cruzada $v$-fold. A validação cruzada generalizada é descrita pelo autor, e consiste em uma aproximação linear do LASSO, substituindo a restrição $\sum_{j}\left|\beta_{j}\right| \leq t$ por $\sum_{j} \frac{\beta_{j}^{2}}{\left|\beta_{j}\right|} \leq t$ e resolvendo o problema por meio de um multiplicador de Lagrange $\lambda$.

Essa resolução, permite, para cada $t$, o cálculo da estatística:

$$
G C V=\frac{1}{n} \frac{S Q R(t)}{\left(1-\frac{p(t)}{n}\right)^{2}}
$$

com:

$$
p(t)=\operatorname{tr}\left(X\left(X^{\prime} X+\lambda W^{-}\right)^{-1} X^{\prime}\right),
$$

na qual $\operatorname{tr}()$ é o operador traço da matriz, $W=\operatorname{diag}\left(\left|\beta_{i}\right|\right)$ e $W^{-}$é uma inversa generalizada de $W$ e $S Q R(t)$ é a soma de quadrados dos resíduos do modelo, dado $t$. Escolhe-se então o valor de $t$ que minimiza $G V C$.

O método de validação cruzada v-fold é descrito em, por exemplo, Breiman e Spector (1992).

Os testes feitos pelo autor indicam que a validação cruzada $v$-fold apresentou resultados melhores na medida de eficácia escolhida pelo autor: minimizar o erro do modelo (EM), definido no mesmo artigo. Muitos dos métodos estudados no presente trabalho são focados em encontrar outros meios de definir o valor $t$. O LARS, tema do Capítulo 2, segue uma abordagem que permite encontrar todas as soluções de LASSO em apenas $p$ passos, sendo assim muito mais simples computacionalmente, enquanto que métodos Bayesianos, estudados no Capítulo 4 entendem $t$ como advinda de uma distribuição de probabilidades à priori (um tema que é sugerido por Tibshirani (1996), mas apenas discutido brevemente). 


\section{Método de Regressão dos mínimos ângulos - LARS}

\subsection{Introdução}

Efron, Hastie, Johnstone e Tibshirani (2004) desenvolvem uma nova metodologia de regressão linear com seleção de variáveis denominada "regressão dos mínimos ângulos"(em inglês Least Angle Regression - LARS), que consiste em criar um vetor de previsão $\hat{\boldsymbol{\mu}}$ de dimensão $m \times 1$, sendo $m$ o número de variáveis explicativas na matriz de planejamento, que é construído em $m$ etapas. Em cada etapa o preditor mais relacionado com a variável explicada é incluído no vetor $\hat{\boldsymbol{\mu}}$, mas não é incluído segundo seu "efeito total"(o que resultaria na solução de mínimos quadrados ordinários), mas sim uma parte desse efeito, calculada de maneira a fazer com que os resíduos sejam igualmente correlacionados com as variáveis anteriormente inclusas e a próxima variável a ser incluída. Então essa próxima variável é incluída e o vetor $\hat{\boldsymbol{\mu}}$ é acrescido não só do efeito da nova variável, mas de um vetor que inclui também uma proporção dos outros preditores incluídos anteriormente. Essa proporção é determinada de acordo com uma interpretação geométrica, segundo a qual o vetor segue uma direção equiangular a todos os preditores não nulos nessa etapa.

Os resultados encontrados pelo LARS são muito similares aos do método LASSO, e uma modificação simples no algoritmo do LARS permite encontrar soluções idênticas a esse método. A metodologia apresentada em Efron et al. (2004) será detalhada nas próximas seções.

\subsection{O algoritmo LARS}

Seja um modelo Linear dado por $\boldsymbol{Y}=X \boldsymbol{\beta}+\boldsymbol{\epsilon}$, com $\boldsymbol{Y}_{n \times 1}$ a variável resposta com $n$ observações, a matriz de planejamento com $m$ variáveis preditoras $X_{n \times m}=\left(\boldsymbol{x}_{1}, \boldsymbol{x}_{2}, \ldots, \boldsymbol{x}_{m}\right)$, com os preditores $\boldsymbol{x}_{1}, \boldsymbol{x}_{2}, \ldots, \boldsymbol{x}_{m}$ linearmente independentes, e o vetor de coeficientes $\boldsymbol{\beta}=\left(\beta_{1}, \beta_{2}, \ldots, \beta_{m}\right)^{\prime}$. Nenhuma estrutura específica é dada ao vetor $\epsilon$. Executa-se a seguir a centralização das variáveis, de maneira que:

$$
\sum_{i=1}^{n} y_{i}=0, \quad \sum_{i=1}^{n} x_{i j}=0, \quad \sum_{i=1}^{n} x_{i j}^{2}=1, \quad j=1, \ldots, m
$$

Será definido o vetor de predição $\hat{\mu}$ :

$$
\hat{\boldsymbol{\mu}}=X \hat{\boldsymbol{\beta}}=\sum_{j=1}^{m} \boldsymbol{x}_{j} \hat{\beta}_{j}
$$

em que $\hat{\beta}_{j}$ é o estimador de $\beta_{j}, j=1,2, \ldots m$.

Além disso, o vetor de resíduos será dado por $(\boldsymbol{Y}-\hat{\boldsymbol{\mu}})$. 
O algoritmo do LARS funciona construindo estimadores $\hat{\beta}_{j}$ em etapas sucessivas, sendo que em cada etapa uma variável é acrescentada ao modelo, e as variáveis acrescentadas anteriormente são ajustadas, de maneira que na etapa $k$, apenas $k \beta_{j}$ 's são diferentes de zero, e no total o processo terá $k=m$ etapas.

Define-se também um vetor para as correlação da etapa atual do processo, $\boldsymbol{c}(\hat{\boldsymbol{\mu}})$, como:

$$
\hat{\boldsymbol{c}}=c(\hat{\mu})=X^{\prime}(\boldsymbol{Y}-\hat{\boldsymbol{\mu}})
$$

Cada um dos $m$ elementos do vetor $\hat{\boldsymbol{c}}$ é denominado $\hat{c}_{j}$ e é proporcional à correlação entre o resíduo atual e o preditor $\boldsymbol{x}_{j}$ (a correlação exata seria dada dividindo-se $\hat{\boldsymbol{c}}$ pelo produto dos desvios padrões de $X$ e de $(\boldsymbol{Y}-\hat{\boldsymbol{\mu}})$, já que as variáveis nos vetores da matriz $X$ foram centralizadas).

O procedimento se inicia especificando o vetor $\hat{\boldsymbol{\mu}}$, definido em (2.1), como nulo, e valores serão acrescentados a ele em etapas, a partir da variável mais correlacionada com o resíduo $(\boldsymbol{Y}-\hat{\boldsymbol{\mu}})$, ou seja, a que apresenta o maior valor de $\hat{c}_{j}$ em (2.2). Para isso deverá ser definido o conjunto "ativo"de variáveis. Seja $\mathcal{A} \subseteq\{1,2, \ldots, m\}$ o subconjunto que contém os índices das variáveis mais correlacionadas com o vetor de resíduos a cada etapa, a matriz "ativa"será:

$$
X_{\mathcal{A}}=\left(\ldots s_{j} \boldsymbol{x}_{j}\right)_{j \in \mathcal{A}}, \quad \text { com } \quad s= \pm 1
$$

Além disso, ainda é necessário definir a proporção na qual cada variável será acrescentada ao vetor $\hat{\mu}$. Isso porque, no LARS, o vetor de predição não recebe imediatamente o "efeito completo"de uma variável $x_{i}$ (que corresponde a $\hat{\beta}_{i}$ no método dos mínimos quadrados), mas somente uma fração desse efeito, que é determinada de acordo com a metodologia descrita a seguir.

A ideia é a seguinte: $\mathrm{O}$ vetor $\boldsymbol{Y}$ é projetado no espaço linear gerado pelas colunas da matriz $X$, que pode ser escrito como $\boldsymbol{v}=a_{1} x_{1}+a_{2} x_{2}+\ldots+a_{m} x_{m}$, no qual $\left\{a_{1}, \ldots, a_{m}\right\}$ são escalares quaisquer (este espaço é denotado por $\mathcal{L}(X)$ ). Encontra-se então o vetor $\boldsymbol{x}_{j}$ mais correlacionado (via (2.2)) com $\boldsymbol{Y}$. Em modelos com variáveis centralizadas, a correlação entre duas variáveis é equivalente ao cosseno do ângulo formado pelos seus dois vetores. Assim, quanto maior a correlação menor o ângulo entre eles. A variável mais correlacionada com $\boldsymbol{Y}$ é a que tem o menor ângulo com esse vetor e será a primeira variável a entrar na formação de $\hat{\boldsymbol{\mu}}$.

Assim, $\hat{\boldsymbol{\mu}}_{1}=\hat{\boldsymbol{\mu}}_{0}+\hat{\gamma}_{1} \boldsymbol{x}_{j}$, no qual $x_{j}$ é a variável mais correlacionada com $Y$ (e lembrando que $\left.\hat{\boldsymbol{\mu}}_{0}=\mathbf{0}\right) \cdot \hat{\gamma_{1}}$ é uma proporção de seu "efeito total"e será definida como a quantidade que leva o vetor de resíduos $(\boldsymbol{Y}-\hat{\boldsymbol{\mu}})$ a ser igualmente correlacionado(ou seja, ter um valor $\hat{c}_{i}$ ) com a próxima variável mais correlacionada, por exemplo $\boldsymbol{x}_{k}$. Geometricamente, isso significa que $\hat{\gamma}_{1}$ é uma quantidade tal que, supondo $\boldsymbol{x}_{j}$ a variável mais correlacionada com $\boldsymbol{Y}$ e $\boldsymbol{x}_{\boldsymbol{k}}$ a segunda mais correlacionada, o ângulo formado pelo vetor $\boldsymbol{Y}$ e $\boldsymbol{x}_{\boldsymbol{j}}$ no espaço $\mathcal{L}(\boldsymbol{X})$ é igual ao ângulo formado por $x_{k}$ e o vetor de resíduos $\left(\boldsymbol{Y}-\hat{\boldsymbol{\mu}_{1}}\right)$.

Utilizando o vetor $\hat{\boldsymbol{\mu}_{1}}$, será construído $\hat{\boldsymbol{\mu}}_{2}$, com $\hat{\boldsymbol{\mu}}_{2}=\hat{\boldsymbol{\mu}}_{1}+\hat{\gamma}_{2} \boldsymbol{u}_{2}$. Nesse caso, o vetor $\hat{\mu}$ não "avança"em direção ao $\boldsymbol{x}_{k}$ (em outras palavras, o vetor não é acrescido de um escalar multiplicado por $\boldsymbol{x}_{\boldsymbol{k}}$ ), mas na direção $\boldsymbol{u}_{2}$, definido a bissetriz unitária do ângulo formado pelas 
duas variáveis mais correlacionadas com o resíduo $^{1}$ (no nosso exemplo $\boldsymbol{x}_{j}$ e $\boldsymbol{x}_{k}$ ). Além disso, $\hat{\gamma}_{2}$ será escolhido de maneira a fazer com que a correlação entre o vetor de resíduos $\left(\boldsymbol{Y}-\hat{\boldsymbol{\mu}}_{2}\right)$ e a próxima variável mais correlacionada sejam iguais entre si. No próximo passo, a direção $\boldsymbol{u}_{3}$ seria um vetor unitário na direção da bissetriz do ângulo formado por $\hat{\boldsymbol{\mu}}_{2}$ e a terceira variável mais correlacionada e assim por diante.

O processo é repetido até que a última variável explicativa seja incluída em $\hat{\boldsymbol{\mu}}_{m}, \mathrm{e} \hat{\gamma}_{m}$ será calculado de maneira a igualar $\hat{\boldsymbol{\mu}}$ à projeção de $\boldsymbol{Y}$ no espaço gerado por $\mathcal{L}\left(\boldsymbol{x}_{1}, \boldsymbol{x}_{2}, \ldots, \boldsymbol{x}_{m}\right)$, que é o resultado de mínimos quadrados ordinários. A figura a seguir clarifica o processo, para o caso de duas variáveis preditoras:

Figura 1 - O algoritmo LARS para dois preditores

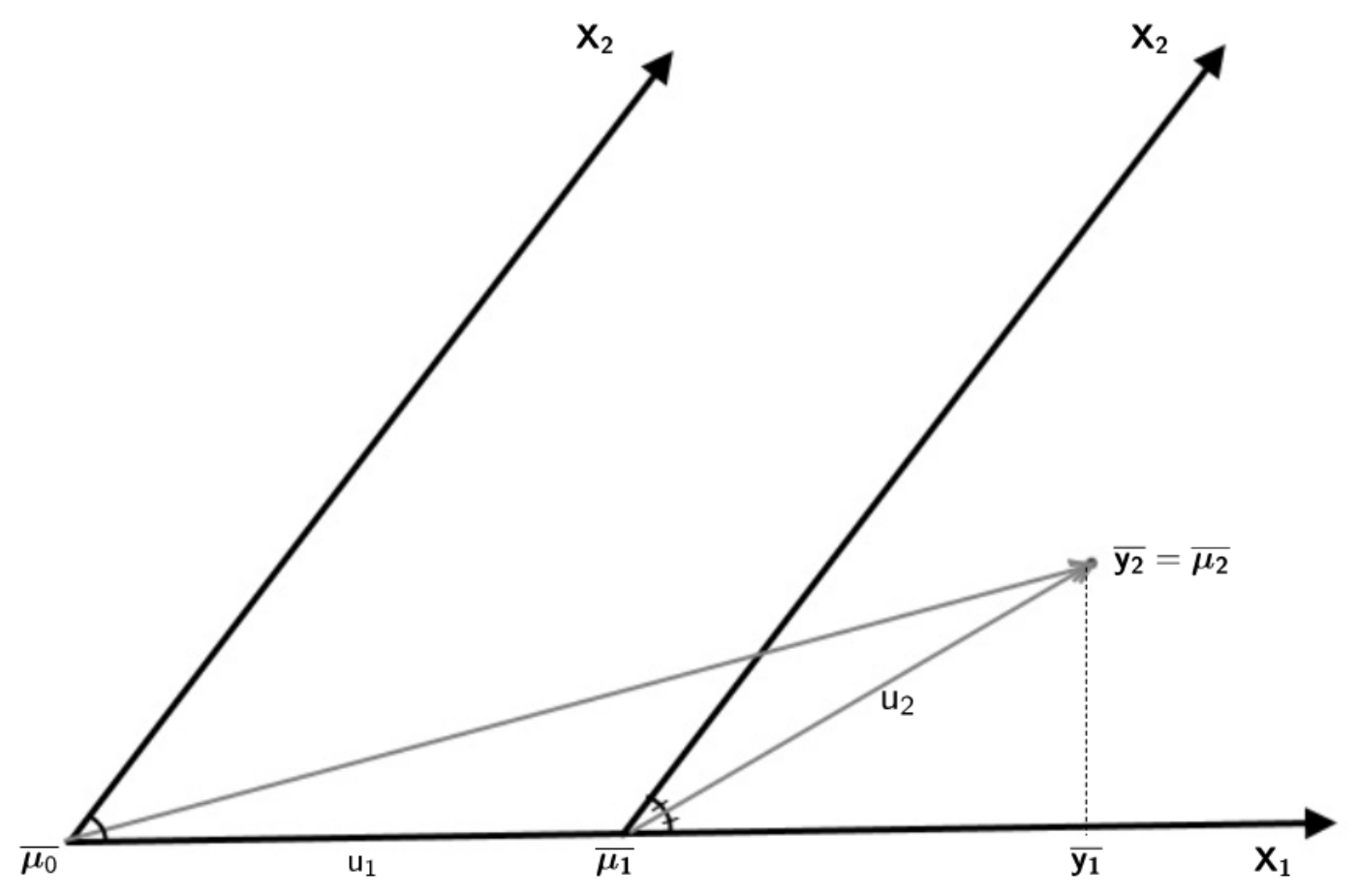

Adaptado de: (EFRON et al., 2004, p. 412)

Na figura, $\overline{\boldsymbol{y}}_{\mathbf{2}}$ é a projeção de $\boldsymbol{Y}$ no espaço gerado por por $\boldsymbol{x}_{\mathbf{1}}$ e $\boldsymbol{x}_{\mathbf{2}}, \mathcal{L}\left(\boldsymbol{x}_{1}, \boldsymbol{x}_{2}\right)$, que representa também a solução de mínimos quadrados ordinários. O ângulo entre o vetor $\boldsymbol{x}_{1}$ e a projeção é menor do que o ângulo entre $\boldsymbol{x}_{2}$ e a projeção, logo $\boldsymbol{x}_{1}$ entrará no cálculo de $\hat{\boldsymbol{\mu}}$ em primeiro lugar. Assim $\hat{\boldsymbol{\mu}}_{1}=0+\hat{\gamma}_{1} \boldsymbol{x}_{1}$.

$\hat{\gamma}_{1}$ é calculado de maneira tal que o novo vetor $\hat{\boldsymbol{\mu}}_{1}$ forme um ângulo com $\overline{\boldsymbol{y}_{\mathbf{2}}}$ igual ao ângulo de $\boldsymbol{x}_{2} \operatorname{com} \overline{\boldsymbol{y}_{\mathbf{2}}}$.

De fato, uma linha que saia da $\hat{\boldsymbol{\mu}}_{1}$ em direção à $\overline{\boldsymbol{y}}$ será a bissetriz do ângulo entre $\hat{\boldsymbol{\mu}}_{1} \mathrm{e}$ $\boldsymbol{x}_{2}$, sendo $\boldsymbol{u}_{2}$ o vetor unitário nessa direção. A próxima etapa é incluir a segunda variável mais

1 a bissetriz unitária é um vetor de comprimento igual a 1 
correlacionada com $\left(\overline{\boldsymbol{y}}_{\mathbf{2}}-\hat{\boldsymbol{\mu}}_{1}\right), \boldsymbol{x}_{2}$. Para isso $\hat{\boldsymbol{\mu}}_{2}=\hat{\gamma}_{2} \boldsymbol{u}_{2}$, ou seja, o vetor de predição vai avançar no sentido da projeção $\overline{\boldsymbol{y}}$ na direção bissetriz $\boldsymbol{u}_{2}$ na proporção $\hat{\gamma}_{2}$. No processo com mais variáveis $\hat{\gamma}_{2}$ seria calculado de maneira a fazer com que uma linha saindo de $\hat{\boldsymbol{\mu}}_{2}$ em direção à projeção de y fosse a bissetriz entre a terceira variável mais correlacionada e $\hat{\boldsymbol{\mu}}_{2}$. Porém, como $\boldsymbol{x}_{2}$ é a última variável, $\hat{\gamma}_{2}$ é calculado de maneira a fazer $\hat{\boldsymbol{\mu}}_{2}=\overline{\boldsymbol{y}_{\mathbf{2}}}$.

Agora é necessário formalizar o processo descrito nos parágrafos anteriores, escrevendo as bissetrizes mencionadas em linguagem matricial e estipulando precisamente o valor de $\hat{\gamma}$.

Sejam:

$$
\mathcal{G}_{\mathcal{A}}=X_{\mathcal{A}}^{\prime} X_{\mathcal{A}} \quad \text { e } \quad A_{\mathcal{A}}=\left(\mathbf{1}_{\mathcal{A}}^{\prime} \mathcal{G}_{\mathcal{A}}^{-1} \mathbf{1}_{\mathcal{A}}\right)^{-\frac{1}{2}}
$$

$\operatorname{com} \mathbf{1}_{\mathcal{A}}$ um vetor de números 1 com uma quantidade de linhas igual a $|\mathcal{A}|$ (o número de elementos do conjunto $\mathcal{A}$ ). Note-se que $A_{\mathcal{A}}$ é um escalar.

Define-se então o vetor equiangular $\boldsymbol{u}_{\mathcal{A}}$ :

$$
\boldsymbol{u}_{\mathcal{A}}=X_{\mathcal{A}} w_{\mathcal{A}}, \quad \operatorname{com} \quad w_{\mathcal{A}}=A_{\mathcal{A}} \mathcal{G}_{\mathcal{A}}^{-1} \mathbf{1}_{\mathcal{A}}
$$

Esse vetor é um vetor unitário que forma ângulos iguais (menores que $90^{\circ}$ ), com os vetores coluna da matriz $X_{\mathcal{A}}$. A demostração desse fato encontra-se no apêndice.

Na construção do algoritmo, também será usado o resultado (obtido diretamente pela aplicação dos resultados (2.3) e (2.4) e demonstrado no apêndice):

$$
X_{\mathcal{A}}^{\prime} \boldsymbol{u}_{\mathcal{A}}=A_{\mathcal{A}} \mathbf{1}_{\mathcal{A}}
$$

Com essas definições, será agora detalhado o processo LARS.

Seja $\hat{\boldsymbol{\mu}}_{\mathcal{A}}$ a estimativa LARS $\boldsymbol{\mu}=X \hat{\boldsymbol{\beta}}$ na etapa atual. Inicia-se com $\hat{\boldsymbol{\mu}}_{0}=\mathbf{0}$, com $\mathbf{0}$ um vetor nulo de dimensão $m \times 1$. Define-se:

$$
\hat{\boldsymbol{c}}=X^{\prime}\left(\boldsymbol{Y}-\hat{\boldsymbol{\mu}}_{\mathcal{A}}\right)
$$

que é denominado vetor das correlações atuais (lembrando que o vetor $\hat{\boldsymbol{c}}$ não representa a correlação propriamente dita, mas é proporcional a ela). Além disso, como $\mathcal{A}$ é um conjunto de índices correspondendo aos preditores que têm as maiores correlações absolutas:

$$
\hat{C}=\max _{j}\left\{\left|\hat{c}_{j}\right|\right\} \quad \text { e } \quad \mathcal{A}=\left\{j:\left|\hat{c}_{j}\right|=\hat{C}\right\}
$$

no qual $\hat{c}_{j}$ são os $j$ elementos do vetor $\hat{\boldsymbol{c}}$. O conjunto $\mathcal{A}$, também chamado de conjunto ativo, começa vazio e na primeira etapa incluí o índice do maior $\left|\hat{c}_{j}\right|$. A partir daí, novos elementos serão adicionados a $\mathcal{A}$ (o índice da variável que possui máxima correlação com os resíduos a cada etapa, tal como mostrado na equação (2.7) ), mas nenhum elemento será removido. Assim, uma vez introduzida uma variável no explicativa, ela jamais sairá do modelo. Essa é uma semelhança do procedimento LARS com algoritmoítmos de seleção do tipo forward. 
Calcula-se então $X_{\mathcal{A}}, A_{\mathcal{A}}$ e $\boldsymbol{u}_{\mathcal{A}}$ tais como definidos anteriormente, tomando $s_{j}=\operatorname{sinal}\left\{\hat{c}_{j}\right\}$. Também é calculado:

$$
a \equiv X^{\prime} u_{\mathcal{A}}
$$

A seguir é feita a atualização do algoritmo:

$$
\hat{\boldsymbol{\mu}}_{\mathcal{A}_{+}}=\hat{\boldsymbol{\mu}}_{\mathcal{A}}+\hat{\gamma} \boldsymbol{u}_{\mathcal{A}}
$$

no qual $\mathcal{A}_{+}=\mathcal{A} \bigcup\{\hat{j}\}$, sendo $\hat{j}$ o novo índice que entra no conjunto ativo( a entrada de um novo índice é garantida a cada etapa, como será provado a seguir). O valor $\hat{\gamma}$ é calculado por:

$$
\hat{\gamma}=\min _{j \in \mathcal{A}^{c}}+\left\{\frac{\hat{C}-\hat{c}_{j}}{A_{\mathcal{A}}-a_{j}}, \frac{\hat{C}+c_{j}}{A_{\mathcal{A}}+a_{j}}\right\} .
$$

No qual $a_{j}$ é o j-ésimo componente de $\boldsymbol{a}$ e $\min ^{+}$significa tomar o mínimo entre os valores positivos das frações apresentadas em (2.10). $\hat{\gamma}$ é calculado de maneira a ser o menor valor positivo tal que um novo índice - $\hat{j}$ - entre no conjunto ativo.

O processo é repetido até a inclusão de todas as variáveis explicativas no conjunto ativo, e no último passo, $M$, define-se $\hat{\gamma}_{M}=\frac{\hat{C}_{M}}{A_{M}}$, com $A_{M}$ definido em $(2.3)$ e $M=1,2 \ldots m$. Este valor de $\hat{\gamma}_{M}$ garante que no último passo, a solução do LARS seja igual à de mínimos quadrados ordinários, como será demonstrado na comparação entre os dois métodos discutida mais a frente.

A prova de que $\hat{\gamma}$ é de fato o menor valor positivo que garante a entrada de um novo índice no conjunto ativo será feita a seguir:

Demonstração. Define-se:

$$
\boldsymbol{\mu}(\gamma)=\hat{\boldsymbol{\mu}}_{\mathcal{A}}+\gamma \boldsymbol{u}_{\mathcal{A}},
$$

com $\gamma>0$. A correlação de uma particular variável explicativa $x_{j}$ com o resíduo do modelo ajustado será dada por:

$$
c_{j}(\gamma)=\boldsymbol{x}_{j}^{\prime}(\boldsymbol{Y}-\boldsymbol{\mu}(\gamma))=\boldsymbol{x}_{j}^{\prime}\left(\boldsymbol{Y}-\hat{\boldsymbol{\mu}}_{\mathcal{A}}\right)-\gamma \boldsymbol{x}_{j}^{\prime} \boldsymbol{u}_{\mathcal{A}}=\hat{c}_{j}-\gamma a_{j}
$$

Para todo $j \in \mathcal{A}$ temos que $\left|\hat{c}_{j}\right|=\hat{C}$. Assim, generalizando para o conjunto $\mathcal{A}$, é possível aplicar esse resultado em (2.5), (2.6) e (2.7) obtendo:

$$
\boldsymbol{c}_{\mathcal{A}}(\gamma)=X_{\mathcal{A}}^{\prime}\left(\boldsymbol{Y}-\mu_{\mathcal{A}}(\gamma)\right)
$$

que leva ao resultado:

$$
\boldsymbol{c}_{\mathcal{A}}(\gamma)=\hat{\boldsymbol{c}}_{\mathcal{A}}-\gamma A_{\mathcal{A}} \mathbf{1}_{\mathcal{A}}
$$

Como está se tratando apenas do conjunto ativo, $\left|\hat{c}_{j}\right|$ será igual a $\hat{C}$ em todos os seus elementos. Lembrando que $A_{\mathcal{A}}$ é um escalar torna-se possível calcular o módulo de cada linha do vetor matriz $\boldsymbol{c}_{\mathcal{A}}$, que será igual a:

$$
\left|c_{j}(\gamma)\right|=\hat{C}-\gamma A_{\mathcal{A}}
$$


Essa equação indica que, fixado o valor de $\gamma$, a correlação de todos elementos incluídos até então em $\mathcal{A}$ com o resíduo declina igualmente.

Tomando um elemento $j \in \mathcal{A}^{c}$ em (2.11) e igualando à (2.14) será encontrado um valor de $\gamma$ que fará com que um $\hat{j}$ fora do conjunto tenha a mesma correlação com o resíduo que todos os elementos dentro do conjunto ativo(lembrando que qualquer $\gamma$ positivo faz com que a correlação dos elementos do conjunto ativo com o resíduo diminua, segundo (2.13)).

Para um $c_{j}$ positivo, é possível igualar (2.11) e (2.14), o que leva ao resultado:

$$
\gamma=\frac{\hat{C}-\hat{c}_{j}}{A_{\mathcal{A}}-a_{j}}
$$

Caso $c_{j}$ seja negativo, $\gamma$ será dado por:

$$
\gamma=\frac{\hat{C}+\hat{c}_{j}}{A_{\mathcal{A}}+a_{j}}
$$

Tomando o mínimo entre os valores positivos desse conjunto, será encontrado o menor $\gamma$ tal que um novo $\hat{j}$ entre no conjunto ativo e isso completa a prova.

O uso do LARS como método de seleção de variáveis.

Cada um dos vetores $\hat{\boldsymbol{\mu}}_{k}$ é um vetor de predição do tipo $\hat{\boldsymbol{\mu}}=X \hat{\boldsymbol{\beta}}$, assim, cada um deles está ligado a um conjunto de estimadores $\hat{\boldsymbol{\beta}}$ para $\boldsymbol{Y}$ e cada um deles têm uma estimativa $\hat{\beta}_{j}$ a mais que o anterior, sendo que o último deles contém betas não-nulos para todos os preditores da matriz de planejamento original. Assim, escolhendo um destes $\hat{\boldsymbol{\mu}}_{k}$ como o modelo final estará se realizando, na prática, uma seleção de variáveis. Os autores propõe uma estatística do tipo $C_{p}$ para selecionar o "melhor" $\hat{\boldsymbol{\mu}}_{j}$, que será apresentada a seguir.

Seja $\hat{\boldsymbol{\mu}}=g(\boldsymbol{Y})$ uma formula criada para estimar $\boldsymbol{\mu}$ a partir de um vetor de dados $\boldsymbol{Y}$. Sejam também os preditores $\boldsymbol{x}_{1}, \ldots, \boldsymbol{x}_{m}$ fixos em seus valores observados. Assume-se que a distribuição de $\boldsymbol{Y}$, condicional aos $\boldsymbol{x}_{j}$ 's é:

$$
\boldsymbol{Y} \sim\left(\boldsymbol{\mu}, \sigma^{2} \boldsymbol{I}\right)
$$

ou seja, os componentes $y_{i}$ de $\boldsymbol{Y}$ são não correlacionados com média $\mu_{i}$ e variância $\sigma^{2}$, mas não assumindo nenhuma distribuição específica para $\boldsymbol{Y}$.

Pode-se escrever $\left(\hat{\mu}_{i}-y_{i}\right)^{2}$ como $\left(\left(\hat{\mu}_{i_{-}} \mu_{i}\right)-\left(y_{i}-\mu_{i}\right)\right)^{2}$, que por sua vez pode ser re-escrita como uma expressão para o erro quadrático da predição:

$$
\left(\hat{\mu}_{i}-\mu_{i}\right)^{2}=\left(y_{i}-\hat{\mu}_{i}\right)^{2}-\left(y_{i}-\mu\right)^{2}+2\left(\hat{\mu}_{i}-\mu_{i}\right)\left(y_{i}-\mu_{i}\right)
$$

Tomando-se essa expressão da identidade, pode-se, em ambos os lados da igualdade, calcular a somatória em $i$, dividir o resultado por $\sigma^{2}$ e calcular a esperança, obtendo-se:

$$
E\left\{\frac{\|\hat{\boldsymbol{\mu}}-\boldsymbol{\mu}\|^{2}}{\sigma^{2}}\right\}=E\left\{\frac{\|\boldsymbol{Y}-\hat{\boldsymbol{\mu}}\|^{2}}{\sigma^{2}}-n\right\}+2 \sum_{i=1}^{n} \frac{\operatorname{cov}\left(\hat{\mu}, y_{i}\right)}{\sigma^{2}} .
$$


A partir de (2.15) pode-se definir os graus de liberdade do estimador $\hat{\boldsymbol{\mu}}=g(\boldsymbol{Y})$ como:

$$
\mathrm{g} l_{\mu, \sigma^{2}}=\sum_{i=1}^{n} \frac{\operatorname{cov}\left(\hat{\mu}, y_{i}\right)}{\sigma^{2}}
$$

a fim de levar a uma estatística do tipo $C_{p}$ dada por:

$$
C_{p}(\hat{\boldsymbol{\mu}})=\frac{\|\boldsymbol{y}-\hat{\boldsymbol{\mu}}\|^{2}}{\sigma^{2}}-n+2 \mathrm{~g} l_{\mu, \sigma^{2}}
$$

Nota-se que esta expressão foi introduzida por (EFRON et al., 2004), sendo diferente do que foi proposto por Mallows (1973), porém, para o caso do modelo linear simples $\hat{\boldsymbol{\mu}}=M \boldsymbol{Y}$ obtém-se $\mathrm{d} f_{\mu, \sigma^{2}}=\operatorname{traço}(M)$, que é igual à definição usual de graus de liberdade para o método dos mínimos quadrados ordinários. Assim, a definição do $C_{p}$ em (2.17) é compatível com a definição original de $C_{p}$ proposta por Mallows.

Estudos de simulação usando bootstrap levaram os autores a propor a seguinte aproximação para os graus de liberdade $\left(\mathrm{g} l_{\mu}\right)$ do LARS:

$$
\mathrm{g} l_{\mu, \sigma^{2}, k} \doteq k
$$

no qual $k$ é o número de variáveis explicativas do modelo, com $k=1,2, \ldots, m$.

Os autores também notam que (2.18) torna-se uma igualdade para os casos em que todos os preditores são ortogonais entre si ou a condição $\mathcal{G}^{-} 1_{\mathcal{A}} \mathbf{1}_{\mathcal{A}}>1$ é cumprida, mas a prova será omitida aqui (ver (EFRON et al., 2004, p. 424 - 426)).

Assim, para realizar a seleção de variáveis, calcula-se (2.17) para cada $\hat{\boldsymbol{\mu}}_{i}$ e o modelo selecionado será aquele que tiver o menor $C_{p}$.

\subsection{Comparação entre o método LARS e o método dos mínimos quadrados ordinários}

Para comparar as estimativas do LARS com as estimativas de mínimos quadrados ordinários (MQO) é necessário examinar mais detalhadamente o processo do LARS como uma projeção ortogonal.

Uma projeção ortogonal de $v$ sobre $u$ é definida como ((LIMA, 2001, p. 127)):

$$
\operatorname{pr}_{u}(\boldsymbol{v})=\frac{\langle\boldsymbol{u}, \boldsymbol{v}\rangle}{\langle\boldsymbol{u}, \boldsymbol{u}\rangle} \cdot \boldsymbol{u},
$$

na qual $\boldsymbol{v}$ é um vetor qualquer e $\boldsymbol{u}$ é um vetor não nulo de mesma dimensão de $\boldsymbol{v}$. O operador $\langle$,$\rangle representa o produto interno dos vetores dentro do operador. Seja \boldsymbol{u}=\left(\alpha_{1}, \alpha_{2}, \ldots, \alpha_{m}\right)$ e $\boldsymbol{v}=\left(\beta_{1}, \beta_{2}, \ldots, \beta_{m}\right)$, o produto interno será dado por $\langle\boldsymbol{u}, \boldsymbol{v}\rangle=\alpha_{1} \beta_{1}+\alpha_{2} \beta_{2}+\ldots+\alpha_{m} \beta_{m}$.

O operador "."representa a multiplicação do escalar resultante por um vetor.

Se dois vetores são perpendiculares, então $\operatorname{pr}_{u}(\boldsymbol{v})=0$. 
Se o vetor $\boldsymbol{v}$ é do tipo $\alpha . \boldsymbol{u}, \operatorname{com} \alpha$ um escalar, então:

$$
\operatorname{pr}_{u}(\boldsymbol{v})=\frac{\langle\boldsymbol{u}, \alpha \cdot \boldsymbol{u}\rangle}{\langle\boldsymbol{u}, \boldsymbol{u}\rangle} \cdot \boldsymbol{u}=\alpha \cdot \frac{\langle\boldsymbol{u}, \boldsymbol{u}\rangle}{\langle\boldsymbol{u}, \boldsymbol{u}\rangle} \cdot \boldsymbol{u}=\alpha \cdot \boldsymbol{u}=\boldsymbol{v},
$$

ou seja, a projeção ortogonal é igual ao vetor original.

Além disso, se $\left(\boldsymbol{z}_{1}, \boldsymbol{z}_{2}, \ldots, \boldsymbol{z}_{m}\right)$ são todos ortogonais entre si, pode-se utilizar o processo de Gram-Schmidt (LIMA, 2001, p. 128) e definir o vetor $\boldsymbol{w}$ como:

$$
\boldsymbol{w}=\sum_{i=1}^{m} \frac{\left\langle\boldsymbol{z}_{i}, \boldsymbol{v}\right\rangle}{\left\langle\boldsymbol{z}_{i}, \boldsymbol{z}_{i}\right\rangle} \cdot \boldsymbol{z}_{i} .
$$

Esse vetor é definido como a projeção de $\boldsymbol{v}$ no espaço gerado pelos vetores $\left(\boldsymbol{z}_{1}, \boldsymbol{z}_{2}, \ldots, \boldsymbol{z}_{m}\right)$ tal espaço será denominado de $\mathcal{L}\left(z_{1}, \ldots, z_{m}\right)$ ou $\mathcal{L}\left(Z_{m}\right)$. Essa mesma projeção também pode ser representada de forma matricial como (JOHNSON; WICHERN, 2007, p. 367 - 369):

$$
\boldsymbol{w}=Z\left(Z^{\prime} Z\right)^{-1} Z^{\prime} \boldsymbol{v}
$$

A matriz $Z\left(Z^{\prime} Z\right)^{-1} Z^{\prime}$ é chamada matriz de projeção, ou matriz chapéu.

O resultado mostrado em (2.20) pode ser estendido para projeções. Se um vetor já pertence ao plano ao qual será projetado, sua projeção será igual ao próprio vetor. Seja:

$$
\boldsymbol{v}=\alpha_{1} \boldsymbol{z}_{1}+\alpha_{2} \boldsymbol{z}_{2}+\ldots+\alpha_{m} \boldsymbol{z}_{m}
$$

um vetor pertencente a $\mathcal{L}\left(Z_{m}\right)$. Então sua projeção será dada por:

$$
\boldsymbol{w}=\sum_{i=1}^{m} \frac{\left\langle\boldsymbol{z}_{i}, \boldsymbol{v}\right\rangle}{\left\langle\boldsymbol{z}_{i}, \boldsymbol{z}_{i}\right\rangle} \boldsymbol{z}_{i}=\sum_{i=1}^{m} \frac{\left\langle\boldsymbol{z}_{i}, \alpha_{1} \boldsymbol{z}_{1}+\ldots+\alpha_{m} \boldsymbol{z}_{m}\right\rangle}{\left\langle\boldsymbol{z}_{i}, \boldsymbol{z}_{i}\right\rangle} . \boldsymbol{z}_{i}
$$

Que pode ser re-escrita como:

$$
\boldsymbol{w}=\sum_{i=1}^{n} \sum_{j=1}^{n} \frac{\left\langle\boldsymbol{z}_{i}, \alpha_{j} \boldsymbol{z}_{j}\right\rangle}{\left\langle\boldsymbol{z}_{i}, \boldsymbol{z}_{i}\right\rangle}=\alpha_{1} \boldsymbol{z}_{1}+\alpha_{2} \boldsymbol{z}_{2}+\ldots+\alpha_{m} \boldsymbol{x}_{m}=\boldsymbol{v}
$$

Isso se dá pois todos os produtos internos do somatório ou recaem no caso (2.20) ou são nulos, devido à ortogonalidade entre os os $x_{i}$.

Na linguagem de modelos lineares, o estimador de mínimos quadrados ordinários é dado por:

$$
\hat{\boldsymbol{Y}}=X \hat{\boldsymbol{\beta}}=X\left(X^{\prime} X\right)^{-1} X^{\prime} \boldsymbol{Y}
$$

Assim, o estimador de MQO pode ser interpretado como a projeção do vetor resposta $\boldsymbol{Y}$ no espaço gerado por $\mathcal{L}\left(X_{m}\right)$.

No caso do LARS, é possível escrever, para cada etapa (de $k=1, \ldots, m$ ) a seguinte identidade:

$$
\overline{\boldsymbol{Y}}=\hat{\boldsymbol{\mu}}_{k-1}+X_{k}\left(X_{k}^{\prime} X_{k}\right)^{-1} X_{k}^{\prime}\left(\boldsymbol{Y}-\hat{\boldsymbol{\mu}}_{k-1}\right)
$$


Na qual, $\overline{\boldsymbol{Y}}$ é a projeção de $Y$ no espaço dado por $\mathcal{L}\left(X_{k}\right)$. Na forma dada por (2.21), (2.24) pode ser escrita como ((LIMA, 2001, p. 130)):

$$
\overline{\boldsymbol{Y}}=\hat{\boldsymbol{\mu}}_{k-1}+\sum_{i=1}^{k} \frac{\left\langle\boldsymbol{x}_{i},\left(\boldsymbol{Y}-\hat{\boldsymbol{\mu}}_{k-1}\right)\right\rangle}{\left\langle\boldsymbol{x}_{\boldsymbol{i}}, \boldsymbol{x}_{\boldsymbol{i}}\right\rangle} \cdot \boldsymbol{x}_{i}
$$

Que, por sua vez, pode ser re-escrita como:

$$
\overline{\boldsymbol{Y}}=\sum_{i=1}^{k} \frac{\left\langle\boldsymbol{x}_{\boldsymbol{i}}, \boldsymbol{Y}\right\rangle}{\left\langle\boldsymbol{x}_{i}, \boldsymbol{x}_{i}\right\rangle} \cdot \boldsymbol{x}_{i}+\hat{\boldsymbol{\mu}}_{k-1}-\sum_{i=1}^{k} \frac{\left\langle\boldsymbol{x}_{\boldsymbol{i}}, \hat{\boldsymbol{\mu}}_{k-1}\right\rangle}{\left\langle\boldsymbol{x}_{i}, \boldsymbol{x}_{i}\right\rangle} \cdot \boldsymbol{x}_{i}
$$

Assim, basta provar que $\hat{\boldsymbol{\mu}}_{k-1}=\sum_{i=1}^{k} \frac{\left\langle\boldsymbol{x}_{i}, \hat{\boldsymbol{\mu}}_{i}\right\rangle}{\left\langle\boldsymbol{x}_{i}, \boldsymbol{x}_{i}\right\rangle} \cdot \boldsymbol{x}_{i}$ para que (2.25) se torne a definição de projeção ortogonal em (2.19), e assim provar a identidade (2.25).

$\hat{\boldsymbol{\mu}}_{k-1}$ é formado recursivamente, tal que:

$$
\hat{\boldsymbol{\mu}}_{k-1}=\sum_{i=1}^{k-1} \hat{\gamma}_{i} \cdot \boldsymbol{u}_{i}
$$

Todos os $\boldsymbol{u}_{i}$ definidos em (2.4) são combinações lineares dos vetores $\left(\boldsymbol{x}_{1}, \ldots, \boldsymbol{x}_{k-1}\right)$, acrescidos dos escalares $\hat{\gamma}_{i}$, logo pertencem ao plano gerado por $\mathcal{L}\left(X_{k-1}\right)$. Assim:

$$
\hat{\boldsymbol{\mu}}_{k-1}=\frac{\left\langle\boldsymbol{x}_{k}, \hat{\boldsymbol{\mu}}_{k-1}\right\rangle}{\left\langle\boldsymbol{x}_{k}, \boldsymbol{x}_{k}\right\rangle} \cdot \boldsymbol{x}_{k}+\sum_{i=1}^{k-1} \frac{\left\langle\boldsymbol{x}_{i}, \hat{\boldsymbol{\mu}}_{i}\right\rangle}{\left\langle\boldsymbol{x}_{i}, \boldsymbol{x}_{i}\right\rangle} \cdot \boldsymbol{x}_{i} .
$$

O primeiro termo do lado direito da igualdade é igual a zero devido à ortogonalidade entre $\hat{\boldsymbol{\mu}}_{k-1}$, uma combinação linear dos vetores $\left(\boldsymbol{x}_{1}, \ldots, \boldsymbol{x}_{k-1}\right)$ e $\boldsymbol{x}_{k}$. O segundo termo é igual à $\hat{\boldsymbol{\mu}}_{k-1}$, o vetor original, devido à (2.23).

Além disso, como todas as correlações correntes na matriz ativa do LARS são iguais a $\hat{C}_{k}$ é possível escrever o vetor de correlações correntes em (2.6) como:

$$
X_{k}^{\prime}\left(\boldsymbol{Y}-\hat{\boldsymbol{\mu}}_{k-1}\right)=\hat{C}_{k} \mathbf{1}_{\mathcal{A}} .
$$

a relação (2.26) pode ser reescrita utilizando-se (2.27), a definição de $A_{\mathcal{A}}$ em (2.3) e a definição de $\boldsymbol{u}$ em (2.4) como:

$$
\hat{\boldsymbol{\mu}}_{k-1}=\frac{\left\langle\boldsymbol{x}_{k}, \hat{\boldsymbol{\mu}}_{k-1}\right\rangle}{\left\langle\boldsymbol{x}_{k}, \boldsymbol{x}_{k}\right\rangle} \cdot \boldsymbol{x}_{k}+\sum_{i=1}^{k-1} \frac{\left\langle\boldsymbol{x}_{i}, \hat{\boldsymbol{\mu}}_{i}\right\rangle}{\left\langle\boldsymbol{x}_{i}, \boldsymbol{x}_{\boldsymbol{i}}\right\rangle} \cdot \boldsymbol{x}_{i}=\hat{\boldsymbol{\mu}}_{k-1}+\frac{\hat{C}_{k}}{A_{k}} \boldsymbol{u}_{k} .
$$

É possível notar a semelhança entre a equação (2.28) e a atualização do LARS descrita em (2.9). Interpretando o método dos mínimos quadrados ordinários na linguagem do LARS pode-se notar que a diferença entre os dois estimadores é que o LARS utiliza o $\hat{\gamma}$, definido em (2.10), enquanto que o MQO, interpretado nos termos propostos pelo LARS, utiliza outro $\gamma$, denominado a partir de agora como $\bar{\gamma}$, dado por:

$$
\bar{\gamma}=\frac{\hat{C}_{k}}{A_{k}}
$$

É por isso que no último passo da atualização do LARS, $\hat{\gamma}$ foi igualado a (2.29). $\hat{\gamma}<\bar{\gamma}$, exceto na última etapa, em que são iguais, por definição. Devido a esse fato, as estimativas do LARS são sempre menores do que as de mínimos quadrados ordinários, exceto na última etapa, em que os estimadores do LARS são iguais aos do MQO. 



\section{Uso de Pseudovariáveis na seleção de va- riáveis nos modelos de regressão.}

\subsection{Introdução}

Luo, Stefanski e Boos (2006) e Wu, Boos e Stefanski (2007) propõe uma abordagem nova nos métodos de seleção de variáveis para modelos de regressão: Utilizar variáveis aleatórias externas e não correlacionadas com as variáveis do modelo original para "calibrar" o nível de significância $\alpha$ utilizado na seleção forward de maneira a encontrar um valor de $\alpha$ "ideal". O procedimento forward depende do estabelecimento de um nível de significância para servir como nível de entrada de variáveis, porem ele não estabelece nenhuma regra para definí-lo. A ideia da calibração é criar uma medida de "eficiência" dos estimadores selecionados por cada nível de significância, de maneira a escolher o "melhor" $\alpha$ para utilizar no procedimento. Luo, Stefanski e Boos (2006) propõe o uso de pseudovariáveis para adicionar "ruído" à variável resposta e, com isso, estimar o viés do erro quadrático médio do modelo, o que por sua vez é utilizado para calibrar $\alpha$. Já Wu, Boos e Stefanski (2007) propõe a inclusão de pseudovariáveis na matriz de planejamento, de maneira a estudar a proporção na qual os métodos de seleção de variáveis incluem variáveis pouco importantes no modelo final e com isso buscar um valor $\alpha$ que mantenha a seleção de preditores pouco importantes em um nível pré-fixado baixo.

\subsection{Método de seleção de modelos com a adição de ruído - NAMS}

O método proposto por Luo, Stefanski e Boos (2006), denominado "Seleção de modelos com a adição de ruído" (cuja sigla em inglês é NAMS), busca estimar o viés do erro quadrático médio de um modelo de regressão adicionando uma perturbação aleatória à variável resposta. Esse método será examinado com detalhes a seguir.

\section{Construção do modelo}

Seja um modelo linear dado por:

$$
\boldsymbol{Y}=\boldsymbol{X} \boldsymbol{\beta}+\boldsymbol{\epsilon}
$$

no qual $\boldsymbol{Y}=\left(Y_{1}, \ldots, Y_{n}\right)^{T}$ é o vetor da variável resposta, $\boldsymbol{\beta}$ o vetor de coeficientes de regressão, de dimensão $p, \boldsymbol{X}_{n \times p}$ uma matriz de planejamento com posto completo com $n$ observações e $p$ variáveis explicativas e $\boldsymbol{\epsilon} \sim N\left(\mathbf{0}, \sigma^{2} \boldsymbol{I}_{n}\right)$, com $\boldsymbol{I}_{n}$ uma matriz identidade de dimensão $n$ e $\sigma^{2}$ desconhecido. Se $J$ é um modelo baseado na regressão de $Y$ em um subconjunto de parâmetros de $\boldsymbol{\beta}$, o erro quadrático médio $(\operatorname{EQM}(J))$ desse modelo será dado por:

$$
\operatorname{EQM}(J)=\frac{\boldsymbol{Y}^{T}\left(\boldsymbol{I}_{n}-\boldsymbol{P}_{J}\right) \boldsymbol{Y}}{n-p_{J}},
$$


com $\boldsymbol{P}_{J}=\boldsymbol{X}_{J}\left(\boldsymbol{X}_{J}^{T} \boldsymbol{X}_{J}\right)^{-1} \boldsymbol{X}_{J}^{T}$ a matriz de projeção de $\boldsymbol{X}_{J}, \boldsymbol{X}_{J}$ a matriz de planejamento do submodelo, sendo que o submodelo $J$ contém $p_{J}$ preditores.

Satisfeitas as suposições básicas do modelo, o erro quadrático médio é o estimador não viesado de $\sigma^{2}$, dado pela soma dos quadrados do resíduo dividido pelo seu número de graus de liberdade. Se o submodelo $J$ contém todas as importantes (ou seja, variáveis cujos coeficientes de regressão são diferentes de zero) para explicar a variável resposta, então $\left(n-p_{J}\right) \times E Q M / \sigma^{2}$ segue uma distribuição $\chi_{n-p_{J}}^{2}$ e $E(E Q M)=\sigma^{2}$ (o estimador EQM é não viesado para $\sigma^{2}$ ). Caso ele omita variáveis importantes $\left(n-p_{J}\right) \times E Q M / \sigma^{2}$ segue uma distribuição $\chi_{n-p_{J}}^{2}$ não central e portanto, $E(E Q M)>\sigma^{2}$. Assim, EQM superestima $\sigma^{2}$. Por outro lado, se o modelo vai na direção contrária e incluí variáveis pouco importantes (ou seja, cujos coeficientes de regressão são iguais a zero), a soma dos quadrados explicados da regressão (SQE) aumenta, o que, por sua vez, causa uma diminuição da soma dos quadrados dos resíduos(SQR), visto que a soma dos quadrados totais(SQT) não se altera com a inclusão ou exclusão de variáveis e SQT = SQE + SQR . Como SQR é utilizada para calcular EQM, neste caso o EQM subestima $\sigma^{2}$.

Partindo destas informações, os autores propõe o uso de um método de SimulationExtrapolation (SIMEX) (COOK; STEFANSKI, 1994), originalmente desenvolvido na literatura de erros de medida, que permite a estimação do viés do estimador erro quadrático médio (EQM).

\section{Inclusão de pseudovariáveis na variável resposta}

Considere-se uma nova variável, $Y^{*}$, obtida a partir da variável $Y$ no modelo (3.1) como sendo:

$$
\boldsymbol{Y}^{*}=\boldsymbol{Y}+\tau \sqrt{\lambda} \boldsymbol{Z}
$$

no qual $\boldsymbol{Z}$ é o vetor de pseudovariáveis independente de $Y$ e que segue uma distribuição $N\left(\mathbf{0}, \boldsymbol{I}_{n}\right)$, com $\tau$ e $\lambda$ estritamente positivos. A nova variável resposta terá os seguintes momentos:

$$
\begin{gathered}
E\left(\boldsymbol{Y}^{*} \mid \boldsymbol{X}\right)=E\left(\boldsymbol{Y}^{\mid} \boldsymbol{X}\right) \\
\operatorname{var}\left(\boldsymbol{Y}^{*} \mid \boldsymbol{X}\right)=\operatorname{var}(\boldsymbol{Y} \mid \boldsymbol{X})+\tau^{2} \lambda \boldsymbol{I}_{n}
\end{gathered}
$$

ou seja, a esperança da nova variável resposta é igual à da antiga, mas a variância de cada termo é acrescida de $\tau^{2} \lambda$, uma quantidade conhecida. Assim, é esperado que as estimativas para a variância de $\boldsymbol{Y}^{*}$ sejam as estimativas para a variância de $\boldsymbol{Y}$ acrescidas de $\tau^{2} \lambda$.

A seguir, uma seleção forward é realizada para construir um modelo baseado no conjunto de dados $\left(\boldsymbol{Y}^{*} ; \boldsymbol{X}\right)$.

Se o nível de significância adotado para a introdução de variáveis explicativas for muito alto, de modo a aceitar variáveis desnecessárias no modelo, o aumento dos estimadores da variância em relação ao modelo real (baseado em $\boldsymbol{Y}$ ) será menor do que o esperado, pois o método será propenso a incluir variáveis não importantes e por consequência disso, $\mathrm{E}(E Q M)<\sigma^{2}$, ou seja, EQM subestima $\sigma^{2}$, como descrito anteriormente. Esse efeito pode ser estudado criando vários conjuntos de dados $\left(\boldsymbol{Y}^{*}, \boldsymbol{X}\right)$, cada um com um diferente valor de $\lambda$ (lembrando que, cada uma dessas variáveis também terá um $\boldsymbol{Z}$ diferente), de modo a gerar uma "grade"de variáveis $\boldsymbol{Y}^{*}$, com valores crescentes de $\lambda$ e mantendo os valores de $\tau$ e $\alpha$ fixos. A seguir, em cada "grade"é 
calculada a inclinação da curva $(\lambda, \mathrm{EQM})$, por meio de uma regressão simples. Se o valor $\alpha$ estiver fixado de maneira a incluir apenas variáveis importantes, não deveria haver viés na estimação de $\sigma^{2}$, e portanto seu valor deveria aumentar linearmente em $\lambda$, com inclinação de $\tau^{2}$. Por outro lado, se o valor de $\alpha$ for alto, de maneira a incluir variáveis pouco importantes, a estimação de $\sigma^{2}$ deve estar viesada para baixo, e portanto, a inclinação da curva será menor do que $\tau^{2}$.

\section{O algoritmo NAMS}

Assim, o procedimento proposto pelos autores consiste em:

1. Gerar vários conjuntos de dados $\boldsymbol{Y}^{*}$ para cada um dos elementos de uma grade composta por valores $\lambda$ tais que:

$$
0<\lambda_{1}<\ldots<\lambda_{m} \leq 4
$$

(ou seja, vários conjuntos independentes para $\lambda_{1}$, vários conjuntos independentes para $\lambda_{2}$, etc.) Esses dados terão a forma: $Y_{i, \lambda_{k}, b}^{*}=Y_{i}+\sqrt{\lambda_{k}} \tau Z_{i, \lambda_{k}, b} \operatorname{com} i=1, \ldots, n ; k=1, \ldots, m ; b=$ $1, \ldots, B$ sendo $b$ o número de réplicas para cada $\lambda_{k}$. Os autores sugerem $B=4000$.

2. Gerar uma grade de valores $\alpha \in[0,1]$ e executar, para cada valor de $\alpha$, uma seleção forward em cada elemento de cada valor da grade de $\lambda^{\prime} s$, calculando, para cada valor de $\lambda$, a média de EQM, denominado $\overline{E Q M}\left(\lambda_{k}, \alpha\right), k=1, \ldots, m$.

3. Para cada $\alpha$ é construida a reta de regressão dos pares $\left(\lambda_{k}, \overline{E Q M}\left(\lambda_{k}, \alpha\right)\right)^{1}$ para $k=1, \ldots, m$, o coeficiente de regressão obtido é indicado por $\hat{\gamma}(\alpha)$.

4. Determinar o valor $\alpha \in(0,1)$ tal que $\hat{\gamma}(\alpha)=\tau^{2}$.

Uma vez encontrado o valor de $\alpha$ no passo 4, este será utilizado como nível de significância "ideal"da seleção forward realizada com o conjunto de dados real. Os autores denominam esse método de NAMS(Noise Addition Model Selection, seleção de modelos com adição de ruído). Também notam que nos casos extremos (valores $\alpha$ iguais a 0 e 1 ), a igualdade $\hat{\gamma}(\alpha)=\tau^{2}$ sempre é verificada, por isso, o objetivo é encontrar $\alpha$ dentro do intervalo aberto. Além disso, também recomendam utilizar $\tau^{2}=\hat{\sigma}_{F}^{2}$, onde $\hat{\sigma}_{F}^{2}$ é dado por $\hat{\gamma}(1)$, que este é um estimador consistente para o erro quadrático médio do modelo completo(pois com o nível de significância 1 todas as variáveis entram).

$\mathrm{Na}$ aplicação do passo quatro do processo descrito é possível que existam vários valores de $\alpha$ para os quais $\hat{\gamma}(\alpha)>\tau^{2}$ (situação que indica uma superestimação, ou viés positivo do estimador, o que sugere um nível de significância tão baixo que exclui variáveis importantes), e também vários valores de $\alpha$ nos quais $\hat{\gamma}(\alpha) \approx \tau^{2}$. Por esse motivo, os autores propõe um método para encontrar o "melhor" $\alpha$.

O valor $\alpha$ "ideal"deveria ser mais alto do que todos os valores de $\alpha$ tais que $\hat{\gamma}(\alpha)$ é maior que $\tau^{2}$ (ou seja, não serão considerados os níveis de significância que excluem variáveis importantes), mas ao mesmo tempo seria "baixo"e geraria um $\hat{\gamma}(\alpha)$ próximo de $\tau^{2}$ (lembrando que os autores fixam $\left.\tau^{2}=\hat{\gamma}(1)\right)$. Esse valor será indicado por $\hat{\alpha}_{*}$, o procedimento para encontrá-lo é:

$\overline{E Q M}=\sum_{b=1}^{B} \frac{E Q M}{B}$ 
1. Definir $\hat{\alpha}_{0}$ como:

$$
\hat{\alpha}_{0}=\max \{\alpha<1: \hat{\gamma}(\alpha)>\hat{\gamma}(1) \text { ou } \hat{\gamma}(\alpha) \text { é máximo local }\}
$$

2. $\hat{\alpha}_{*}$ é o menor valor de $\alpha$ tal que $\hat{\alpha}_{*}>\hat{\alpha}_{0}$.

Os autores também apresentam uma figura para ilustrar melhor o método:

Figura 2 - Procedimento para determinação de $\hat{\alpha}_{*}$

Caso $a$

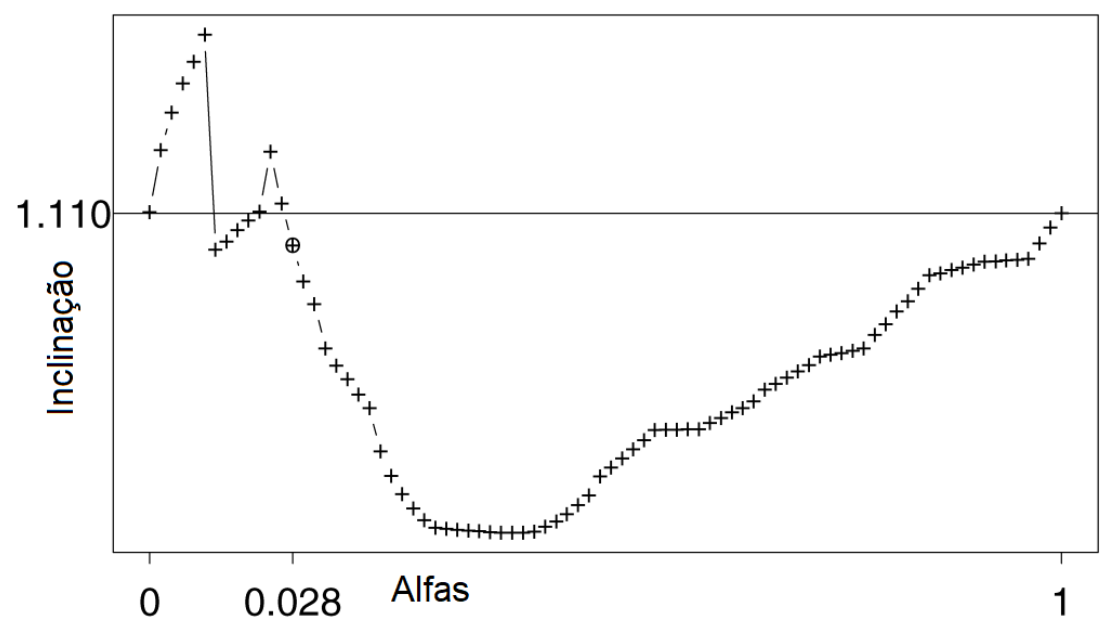

Caso $b$

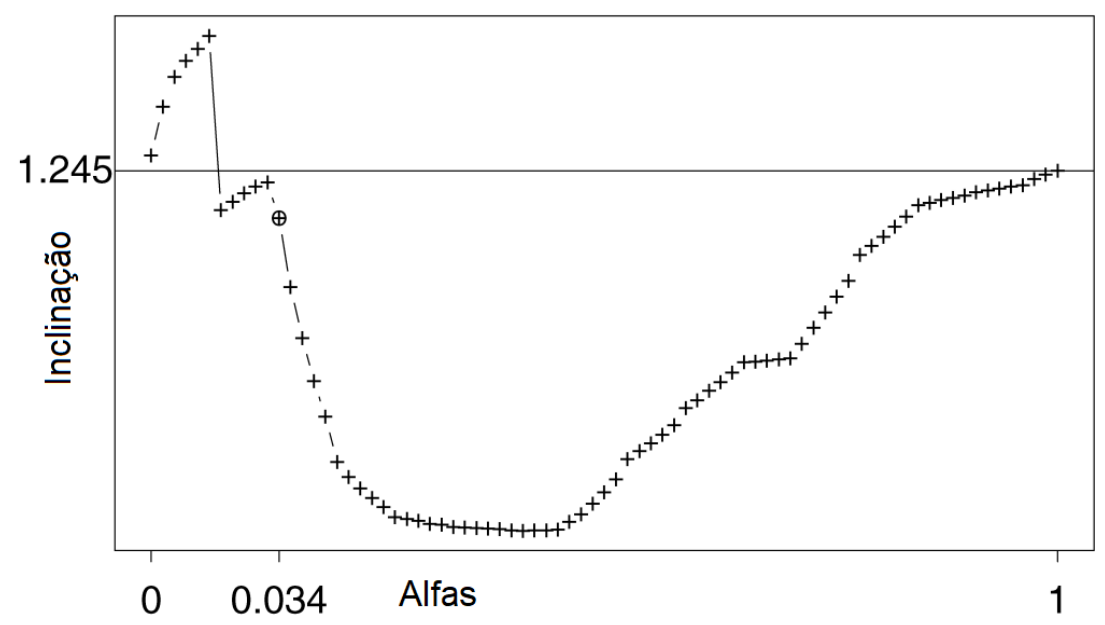

Fonte: (LUO; STEFANSKI; BOOS, 2006, p. 6), traduzido

Caso $a: \tau^{2}=1,110$, Caso $b: \tau^{2}=1,245$

Nos gráficos apresentados na Figura 1 tem-se a inclinação $\hat{\gamma}(\alpha)$ no eixo das ordenadas e os valores de $\alpha$ no eixo das abscissas, com a linha representando EQM $(\hat{\gamma}(1))=\tau^{2}$. Os símbolos de + estão posicionados nos pontos $(\alpha, \hat{\gamma}(\alpha))$ para cada um dos valores de $\alpha$ contidos na grade de $\alpha$ 's criada anteriormente e a abscissa do ponto associado ao símbolo $\oplus$ é o valor de $\alpha$ escolhido pelo método descrito. No caso $a, \oplus$ foi escolhido por estar associado ao valor de $\alpha$ que vem logo 
a seguir do último $\alpha$ a cruzar a linha do $\hat{\gamma}(1)$. No caso $b$ ele é escolhido por ser o primeiro $\alpha$ após o último máximo local de $\hat{\gamma}(\alpha)$.

\section{Conclusão}

Os autores fazem alguns estudos de simulação comparando o NAMS com outros métodos de seleção de variáveis como o Little Bootstrap, o $C_{p}$ de Mallows e o $C_{p}$ "inteligente"(MALLOWS, 1995), e chegam à conclusão de que, em comparação com esses métodos, o NAMS funciona melhor quando o número de variáveis importantes é baixo (até 10) e com baixa correlação entre si(quando a correlação é maior que 0,7 , os três métodos citados acima forneceram estimativas melhores). Além disso, o NAMS também se tornou relativamente melhor que os outros métodos em modelos com mais variáveis explicativas inciais. Um outro resultado importante é que o NAMS também é o método que produziu a maior redução no tamanho do modelo (isto é, no número de variáveis explicativas incluídas no modelo) em todos os casos estudados.

\subsection{O método da razão de falsa seleção}

Wu, Boos e Stefanski (2007) propõe um método de seleção do tipo Forward baseado na inclusão de pseudovariáveis - variáveis intencionalmente não-explicativas e não correlacionadas com a variável resposta. A ideia principal do artigo é a de que um modelo selecionado por métodos automatizados de seleção de variáveis em geral incluirá tanto variáveis informativas quanto não informativas. Um modelo bem selecionado deveria incluir a maioria das variáveis informativas da amostra e poucas variáveis não-informativas. Assim, um modelo pode ser, na nomenclatura dos autores overfitted (com tendência a incluir preditores a mais do que o necessário) ou underfitted (com tendência a excluir variáveis informativas pouco importantes). A inclusão de pseudovariáveis permitirá não somente descobrir em qual das duas categorias o modelo em estudo se encaixa, mas também como definir um nível de significância de entrada de variáveis $\alpha$ de maneira a controlar, em média, o percentual de variáveis não informativas contido no modelo final.

A seguir o método de Wu, Boos e Stefanski (2007) será detalhado.

\section{Construção do Modelo}

Seja o um modelo de regressão qualquer definido por:

$$
E\left(\boldsymbol{Y} \mid X_{1}, \ldots, X_{k_{T}}\right)=g\left(\beta_{0}+\beta_{1} X_{1}+\ldots+\beta_{k_{T}} X_{k_{T}}\right),
$$

com $\boldsymbol{Y}_{\boldsymbol{n} \times \mathbf{1}}, X_{i}, i=1, \ldots, k_{T}$ e $\boldsymbol{X}_{\boldsymbol{n} \times \boldsymbol{k}_{\boldsymbol{T}}}$ a matriz de planejamento. $k_{T}$ é o número total de variáveis ( $T$ é o número total de variáveis na base de dados real), sendo $k_{I}$ o número de variáveis informativas e $k_{N I}$ o número de variáveis não informativas, de modo $k_{T}=k_{I}+k_{N I}$. Uma variável é considerada informativa se $\left|\beta_{j}\right| \neq 0$ e não-informativa caso contrário. São agora formados $B$ conjuntos de dados aumentados. Em cada um deles acrescenta-se $k_{P}$ pseudovariáveis, que formarão matrizes $\boldsymbol{Z}_{\boldsymbol{b}}, b=1, \ldots, B$, cada uma delas de dimensão $n \times k_{P}, \operatorname{com} \boldsymbol{Z}_{\boldsymbol{b}}=\left(Z_{b 1}, Z_{b 2}, \ldots, Z_{b k_{P}}\right)$ de modo a obter $B$ conjuntos de dados da forma $\left(\boldsymbol{Y} ; \boldsymbol{X}, \boldsymbol{Z}_{\boldsymbol{b}}\right)$ com $b=1, \ldots, B$, cada matriz de planejamento aumentada terá dimensão $n \times\left(k_{T}+k_{P}\right)$. 
A seguir, define-se, para cada modelo em $(\boldsymbol{Y} ; \boldsymbol{X}), \operatorname{com} N I(\boldsymbol{Y} ; \boldsymbol{X})$ e $I(\boldsymbol{Y} ; \boldsymbol{X})$ sendo o número de variáveis não informativas e informativas, respectivamente, no modelo final a fração $\gamma(\boldsymbol{Y} ; \boldsymbol{X})$ :

$$
\gamma(\boldsymbol{Y} ; \boldsymbol{X})=\frac{N I(\boldsymbol{Y} ; \boldsymbol{X})}{1+N I(\boldsymbol{Y} ; \boldsymbol{X})+I(\boldsymbol{Y} ; \boldsymbol{X})}
$$

na qual o denominador representa o número total de variáveis no modelo final (informativas mais não informativas e o intercepto).

Os autores tratam $N I(\boldsymbol{Y} ; \boldsymbol{X})$ e $I(\boldsymbol{Y} ; \boldsymbol{X})$ como variáveis aleatórias (ou seja, o número de variáveis importantes e não importantes selecionadas para o modelo final, após a aplicação do método forward serão consideradas variáveis aleatórias, pois dependem da amostragem), mas não atribuem nenhuma distribuição de probabilidades específica para elas, apenas consideram que sua esperança é positiva e finita. Por consequência, $\gamma(\boldsymbol{Y} ; \boldsymbol{X})$ também é considerada uma variável aleatória com esperança positiva e finita.

A expressão (3.2) pode ser reescrita como:

$$
N I(\boldsymbol{Y} ; \boldsymbol{X})-\{1+N I(\boldsymbol{Y} ; \boldsymbol{X})+I(\boldsymbol{Y} ; \boldsymbol{X})\} \gamma(\boldsymbol{Y} ; \boldsymbol{X})=0 .
$$

O método desenvolvido a seguir tem por objetivo fazer com que os modelos selecionados por meio de uma aplicação do método forward tenham, aproximadamente, um valor de $\gamma(\boldsymbol{Y} ; \boldsymbol{X})$ igual a um nível pré-determinado, que representa a tolerância para razão de variáveis não importantes presentes no modelo final em relação ao total de variáveis incluídas no modelo final (por exemplo 0,05). Esse grau de tolerância foi denominado $\gamma_{0}$. A partir das expressões (3.2) e (3.3) é possível aplicar o operador esperança, e fixando essa esperança como o valor de $\gamma_{0}$ desejado, são propostos:

$\mathrm{ou}$

$$
\gamma_{0}=E\left\{\frac{N I(\boldsymbol{Y} ; \boldsymbol{X})}{1+N I(\boldsymbol{Y} ; \boldsymbol{X})+I(\boldsymbol{Y} ; \boldsymbol{X})}\right\}
$$

$$
\gamma_{0}=\frac{E\{N I(\boldsymbol{Y} ; \boldsymbol{X})\}}{E\{1+N I(\boldsymbol{Y} ; \boldsymbol{X})+I(\boldsymbol{Y} ; \boldsymbol{X})\}} .
$$

As expressões (3.4) e (3.5) apresentam duas abordagens diferentes para o problema: Em (3.4), $\gamma_{0}$, representa o valor esperado da variável aleatória $\gamma(\boldsymbol{Y} ; \boldsymbol{X})$, enquanto em (3.4) seria a razão das esperanças. Apesar de partirem de pressupostos diferentes, os estudos de simulação conduzidos pelos autores mostram que ambas as abordagens levam a resultados muito próximos.

\section{Os métodos da razão de falsa seleção}

Os métodos apresentados a seguir - chamados de métodos da razão de falsa seleção consistem em encontrar um nível de significância $\alpha$ que mantenha a medida $\gamma_{E R}$ ou $\gamma_{R E}$ próximas de um nível $\gamma_{0}$ pré-determinado.

Para isso, foi utilizado o fato de que o número de variáveis inclusas no modelo final é função do nível de significância, de maneira que teremos $S(\alpha)=I(\alpha)+N I(\alpha)$ (S, I e NI, também são função de $\boldsymbol{Y}$ e $\boldsymbol{X}$, mas essas variáveis serão omitidas na notação para maior clareza). 
Assim:

$$
\gamma_{E R}=E\left\{\frac{N I(\alpha)}{1+S(\alpha)}\right\}=E\left\{\frac{N I(\alpha)}{1+N I(\alpha)+I(\alpha)}\right\}
$$

e

$$
\gamma_{R E}=\frac{E\{N I(\alpha)\}}{E\{1+S(\alpha)\}}=\frac{E\{N I(\alpha)\}}{E\{1+I(\alpha)+N I(\alpha)\}} .
$$

O procedimento consiste em encontrar $\alpha_{*}=\sup _{\alpha}\left\{\alpha: \gamma_{R} E\left(\alpha_{*}\right) \leq \gamma_{0}\right\}$, ou $\alpha_{*}=\sup _{\alpha}\{\alpha$ : $\left.\gamma_{E} R\left(\alpha_{*}\right) \leq \gamma_{0}\right\}$, dependendo da abordagem desejada.

\section{Método baseado na razão das esperanças}

Seja $S_{P, b}(\alpha)$ o número total de variáveis selecionadas em um método forward de um modelo de regressão baseado em $\left(\boldsymbol{Y} ; \boldsymbol{X}, \boldsymbol{Z}_{\boldsymbol{b}}\right)$ (um modelo de regressão com a matriz de dados com os regressores e pseudoregressores), $S_{P, b}(\alpha)=I_{P, b}(\alpha)+N I_{P, b}(\alpha)+N I_{P, b}^{*}$, com $I_{P, b}$ e $N I_{P b}$ o número de variáveis informativas e não informativas no modelo final e $N I_{P, b}^{*}$ o número de pseudovariáveis presentes no modelo final (o $\mathrm{P}$ representa o total de variáveis, variáveis informativas e não informativas selecionadas pelo modelo depois da inclusão de pseudovariáveis). É importante notar que, em princípio, não é possível distinguir $I_{P, b}(\alpha)$ e $N I_{P, b}(\alpha)$, porém a quantidade $N I_{P, b}^{*}$ se torna conhecida após a seleção forward ser executada, bem como $S_{P, b}(\alpha)$.

Neste momento, é possível estimar $\gamma_{R E, P}(\alpha)$, a razão da esperança da quantidade de pseudovariáveis presentes no modelo final em relação ao total de variáveis inclusas no modelo,

$$
\gamma_{R E, P}(\alpha)=\frac{E\left\{N I_{P, b}^{*}(\alpha)\right\}}{E\left\{1+S_{P, b}(\alpha)\right\}}=\frac{E\left\{N I_{P, b}^{*}(\alpha)\right\}}{E\left\{1+I_{P, b}(\alpha)+N I_{P, b}(\alpha)+N I_{P, b}^{*}(\alpha)\right\}} .
$$

Como anteriormente haviam sido construídos $B$ conjuntos de dados aumentados, podemos calcular a média do total de variáveis selecionadas nos $B$ modelos e também a média do número de pseudovariáveis inclusas no modelo, por:

$$
\bar{S}_{P}(\alpha)=B^{-1} \sum_{b=1}^{B} S_{P, b}(\alpha) .
$$

e

$$
\overline{N I}_{P}^{*}(\alpha)=B^{-1} \sum_{b=1}^{B} N I_{P, b}^{*}(\alpha) .
$$

Observa-se que, para B suficientemente grande, pode-se aplicar a Lei dos Grandes Números e construir o estimador consistente:

$$
\hat{\gamma}_{R E, P}(\alpha)=\frac{\overline{N I}_{P}^{*}(\alpha)}{1+\bar{S}_{P}(\alpha)}
$$

Assim obtém-se a estimativa da proporção da esperança do número de pseudovariáveis incluídas no modelo, em relação à esperança do número de variáveis totais. O objetivo, porém 
não é estimar a proporção de pseudovariáveis no modelo final, mas a proporção de variáveis não informativas, ou, equivalentemente, o valor de $\alpha_{*}$. Para fazer a ligação entre a relação (3.9) e a quantidade desejada será necessária a inclusão de duas hipóteses:

$$
\begin{gathered}
H 1: E\{N I(\alpha)\}=E\left\{N I_{P, b}(\alpha)\right\}=E\left\{N I_{P, b}^{*}(\alpha)\right\} k_{N I} / k_{P}, \Rightarrow \frac{E\left\{N I_{P, b}(\alpha)\right\}}{E\left\{N I_{P, b}^{*}(\alpha)\right\}}=\frac{k_{N I}}{k_{P}} \\
H 2: E\left\{I_{P, b}(\alpha)\right\}=E\{I(\alpha)\} .
\end{gathered}
$$

A primeira igualdade em (H1) significa que em média, o número de variáveis não informativas presentes no modelo final é independente da inclusão ou não de pseudovariáveis. A segunda igualdade determina que em média, o número de variáveis não informativas selecionadas no modelo em relação ao total de variáveis não informativas na matriz de planejamento original será igual ao número de pseudovariáveis selecionadas no modelo, em relação ao total de pseudovariáveis incluídas nas matrizes de dados aumentados. Ou seja, a seleção forward selecionará a mesma proporção (em relação ao total dessas variáveis) de variáveis não informativas e pseudovariáveis. A hipótese (H2) significa que, em média, o número de variáveis informativas presentes no modelo final independe da inclusão de pseudovariáveis.

Utilizando essas duas hipóteses é possível obter a relação entre $S(\alpha)$ e $S_{P, b}(\alpha)$ :

$$
\begin{gathered}
E(S(\alpha))=E\left(I(\alpha)+E(N I(\alpha)) \stackrel{H 1 e H 2}{\Rightarrow} E(S(\alpha))=E\left(N I_{P, b}(\alpha)\right)+E\left(I_{P, b}(\alpha)\right)\right. \\
S_{P, b}(\alpha)=E\left(N I_{P, b}(\alpha)\right)+E\left(I_{P, b}(\alpha)\right)+E\left(N I_{P, b}^{*}(\alpha)\right)
\end{gathered}
$$

Logo,

$$
E(S(\alpha))=S_{P, b}(\alpha)-E\left(N I_{P, b}^{*}(\alpha)\right)
$$

Fixando $\gamma_{R E}(\alpha)=\gamma_{0}$ em (3.7), utilizando (3.10) e (H1) na relação (3.5) obtêm-se:

$$
\gamma_{R E}=\gamma_{0}=\frac{E\left\{N I_{P, b}^{*}(\alpha)\right\} \frac{k_{U}}{k_{P}}}{1+S_{P, b}(\alpha)-E\left\{N I_{P, b}^{*}(\alpha)\right\}} \Rightarrow E\left\{N I_{P, b}^{*}(\alpha)\right\}=\frac{\gamma_{0}\left(1+S_{P, b}(\alpha)\right)}{\gamma_{0}+\frac{k_{N} I}{k_{P}}},
$$

que substituído em (3.8) resulta em:

$$
\gamma_{R E, P}(\alpha)=\frac{\gamma_{0}}{\gamma_{0}+\frac{k_{N I}}{k_{P}}}
$$

fixando $\gamma_{R E, P}(\alpha)=c$ e reorganizando os termos obtém-se:

$$
c=\frac{\gamma_{0} k_{P}}{\gamma_{0} k_{P}+k_{N I}} .
$$

Substituindo $c$ pelo estimador (3.9) e isolando $\gamma_{0}$ obtém-se:

$$
\frac{\overline{N I}_{P}^{*}(\alpha) k_{N I} / k_{P}}{1+\bar{S}_{P}(\alpha)-\overline{N I}_{P}^{*}(\alpha)}=\gamma_{0}
$$


Utilizando as hipóteses $\mathrm{H} 1$ e H2, percebe-se que $\overline{N I}_{P}^{*}(\alpha) k_{N I} / k_{P}$ estima $E\{N I(\alpha)\}$ e $1+\bar{S}_{P}(\alpha)-\overline{N I}_{P}^{*}(\alpha)$ estima $E\{1+I(\alpha)+N I(\alpha)\}$. Logo, este é um estimador para $\gamma_{R E}(\alpha)$. Porém, a quantidade $k_{N I}$ é desconhecida. Para contornar esse problema define-se:

$$
\hat{k}_{N I}(\alpha)=k_{T}-S(\alpha)
$$

Como espera-se que a proporção de variáveis não informativas no modelo seja pequena, admite-se que:

$$
\hat{k}_{N I} \approx k_{N I}
$$

Substituindo $k_{N I}$ por $\hat{k}_{N I}$ em (3.11) pode-se definir o estimador:

$$
\hat{\gamma}_{R E}(\alpha)=\frac{\bar{U}_{P}^{*}(\alpha) \hat{k}_{U} / k_{P}}{1+\bar{S}_{P}(\alpha)-\bar{U}_{P}^{*}(\alpha)} .
$$

Observa-se que esse estimador não é ideal para valores de $\alpha$ muito altos pois $\hat{\gamma}_{R E}(1)=0$ (o que significa que, quando a probabilidade do erro tipo 1 no teste de significância é de 100 \% o estimador indica que não há nenhuma variável não informativa, o que não é razoável). Os autores recomendam utilizar $\alpha_{M A X}=0,2$. Assim, fixado $\gamma_{0}$ e obedecendo o limite do $\alpha_{M A X}$, o nível de significância que mantém a razão das esperanças desejada é dado por:

$$
\hat{\alpha}_{R E}=\sup _{\alpha \leq \alpha_{M} A X}\left\{\alpha: \hat{\gamma}(\alpha) \leq \gamma_{0}\right\}
$$

Nas aplicações práticas, $\hat{\alpha}_{R E}$ é obtido criando uma grade finita de valores $\alpha$, aplicando $\hat{\gamma}(\alpha)$ a cada um deles e tomando o $\alpha$ máximo que respeite $\hat{\gamma}(\alpha) \leq \gamma_{0}$.

Uma vez determinado $\hat{\alpha}_{R E}$ obtém-se o modelo final aplicando o procedimento forward com nível de entrada $\hat{\alpha}_{R E}$

\section{Método baseado na Esperança da Razão}

Utilizando a mesma nomenclatura da sessão anterior temos de estimar:

$$
E\left\{\frac{N I(\alpha)}{1+S(\alpha)}\right\}
$$

Nesse caso será adotada uma estratégia diferente, em vez de estimar $\gamma_{E R, P}$ e encontrar sua relação com $\gamma_{E R}$ será utilizado o fato de que $S(\alpha)$, dado $\alpha$ é conhecido (ele é puramente o número de variáveis no modelo final para cada nível de significância na regressão sem os dados aumentados) e que, pela sessão anterior podemos construir um estimador de $U(\alpha), \hat{N I}(\alpha)=\frac{\hat{k}_{N I}}{k_{P}} U_{P}^{*}(\alpha)$. Assim, o estimador de $\gamma_{R E}(\alpha)$ será dado por:

$$
\hat{\gamma}_{E R}(\alpha)=\frac{\overline{N I}_{P}^{*}(\alpha) \hat{k}_{N I}(\alpha) / k_{P}}{1+S(\alpha)}
$$

e o valor de alfa que leva à esperança da razão desejada é dado por:

$$
\hat{\alpha}_{E} R=\sup _{\alpha \leq \alpha_{M A X}}\left\{\alpha: \hat{\gamma}_{E R} \leq \gamma_{0}\right\}
$$




\section{Geração de Pseudovariáveis}

Os autores sugerem quatro métodos para a geração de pseudovariáveis (WU; BOOS; STEFANSKI, 2007, p. 238):

1. Os elementos na matriz $\boldsymbol{Z}$ de dimensão $n \times k_{P}$ são gerados a partir de distribuições Normais $(0,1)$, independentes, identicamente distribuídas,

2. as $n$ linhas de $\boldsymbol{Z}$ são obtidas a partir da permutação aleatória das linhas de $\boldsymbol{X}$ (o que restringiria a $k_{t}=k_{P}$ ). Como as medidas relevantes ao indivíduo 1 são irrelevantes para o indivíduo 2, a permutação aleatória gera variáveis que podem ser consideradas pseudovariáveis para aquele indivíduo (por exemplo, em um estudo médico, as medições de sangue de um indivíduo não deveriam ter relação alguma com a condição clínica de outro indivíduo).

3. a matriz $\boldsymbol{Z}$ obtida pelo método 1 é substituída por $\left(\boldsymbol{I}-\boldsymbol{H}_{\boldsymbol{X}}\right) \boldsymbol{Z}, \operatorname{com} \boldsymbol{H}_{\boldsymbol{X}}=\boldsymbol{X}\left(\boldsymbol{X}^{T} \boldsymbol{X}\right)^{-1} \boldsymbol{X}^{T}$ (a "matriz chapéu"de $\boldsymbol{X}$ ).

4. a matriz $\boldsymbol{Z}$ obtida pelo método 2 é substituída por $\left(\boldsymbol{I}-\boldsymbol{H}_{\boldsymbol{X}}\right) \boldsymbol{Z}$, com $\boldsymbol{H}_{\boldsymbol{X}}=\boldsymbol{X}\left(\boldsymbol{X}^{T} \boldsymbol{X}\right)^{-1} \boldsymbol{X}^{T}$.

Os quatro métodos produzem variáveis estocásticamente não correlacionadas com $\boldsymbol{X}$, com a vantagem de que a permutação realizada nos métodos 2 e 4 gera pseudovariáveis com os mesmos momentos amostrais das variáveis preditoras e, se houver correlação entre variáveis em $\boldsymbol{X}$, essa correlação se manterá em $\boldsymbol{Z}$. Segundo os estudos de simulação dos autores, o uso da matriz $\boldsymbol{H}_{\boldsymbol{X}}$ tende a reduzir a exclusão de variáveis preditoras importantes devido à inclusão das pseudovariáveis (auxiliando na verificação da hipótese 2). Após os estudos de simulação, os autores recomendam o uso do método quatro.

Além disso, os estudos de simulação também compararam a performance dos métodos da razão de falsa seleção, RFS (FSR em inglês), com o NAMS, discutido anteriormente, além dos conhecidos métodos de Little Bootstap, Lasso e $C_{P}$. Para essa comparação foi encontrada a "melhor"seleção de variáveis, com base no conhecimento do modelo verdadeiro usado na simulação, e calculado o "erro do modelo (ER)", definido no próprio artigo (WU; BOOS; STEFANSKI, 2007, p. 236). Calculou-se, a seguir, a razão entre o erro do modelo obtido por cada um dos métodos e o ER do "melhor"modelo (conhecido a priori). Em modelos com poucas variáveis explicativas importantes (até 6) e nenhuma correlação, o FSR e o NAMS obtiveram modelos melhores que os outros métodos, com o NAMS tendo uma pequena vantagem sobre o FSR. Com uma correlação mais alta $(0,7)$ e 6 ou mais variáveis importantes, o LASSO se mostrou melhor do que o FSR. No quesito redução do tamanho do modelo, os métodos NAMS e RFS tiveram resultados semelhantes, com uma redução muito maior do que dos outros métodos, sendo que o LASSO produz modelos muito maiores do que todos os métodos comparados. Também foi calculada a razão de falsa seleção do FSR (com o conhecimento do número verdadeiro de variáveis importantes) e a simulação mostrou que, de fato, os métodos FSR mantém a razão de falsa seleção próxima do desejado. 


\subsection{Conclusão}

Os dois métodos possuem em comum o fato de que utilizam pseudovariáveis externas aos modelos para "calibrar"o valor de $\alpha$ - nível de entrada - da seleção forward, mas possuem abordagens diferentes: Luo, Stefanski e Boos (2006) adicionam pseudovariáveis na forma de ruído à variável resposta, enquanto Wu, Boos e Stefanski (2007) adicionam pseudovariáveis como preditores na matriz de planejamento. Ambos também o fazem por motivos diferentes: o primeiro para estimar o viés do erro quadrático médio e o segundo para estimar uma nova medida proposta no próprio artigo - a razão de falsa seleção.

Apesar de partirem de abordagens diferentes, os estudos de simulação apresentados nos dois artigos mostram que, em termos de aplicação prática, os dois métodos servem a propósitos semelhantes: São melhores em modelos com pouca ou nenhuma correlação entre preditores e com poucas variáveis explicativas importantes. Além disso, se o objetivo do pesquisador for obter modelos mais parcimoniosos sem incorrer em um grande aumento no erro do modelo (ER), tanto o NAMS quanto o RSF se mostram melhores do que vários dos métodos tradicionais. 



\section{Os métodos de LASSO Bayesianos para se- leção de variáveis}

\subsection{Introdução}

Desde que foi proposto em Tibshirani (1996) o procedimento LASSO tornou-se um dos métodos mais populares de seleção de variáveis, pela simplicidade, velocidade e esparsidade. Ao mesmo tempo, a inferência bayesiana está se tornando uma abordagem cada vez mais popular entre os estatísticos. É natural, então, que nos últimos anos tenha-se buscado conciliar a metodologia do LASSO com a abordagem bayesiana. Este Capítulo discute algumas dessas abordagens.

\subsection{Seleção de variáveis via Modelos Hierárquicos e o procedimento Bayes empírico}

Yuan e Lin (2005) propõe o uso de um modelo bayesiano hierárquico para efetuar a seleção de variáveis, utilizando uma distribuição de Laplace como priori,com estimação por maximização da probabilidade à posteriori que, em determinadas circunstâncias, corresponde exatamente ao procedimento LASSO.Propõe ainda um procedimento dentro da metodologia de Bayes empírico para definir os parâmetros do LASSO.

\subsubsection{Introdução}

Seja um modelo Linear dado por $\boldsymbol{Y}=X \boldsymbol{\beta}+\boldsymbol{\epsilon}$, com $\boldsymbol{Y}_{n \times 1}$ o vetor de valores da variável resposta com $n$ observações, a matriz de planejamento com $p$ variáveis preditoras $X_{n \times p}=$ $\left(\boldsymbol{x}_{1}, \boldsymbol{x}_{2}, \ldots, \boldsymbol{x}_{p}\right)$, com os preditores $\boldsymbol{x}_{1}, \boldsymbol{x}_{2}, \ldots, \boldsymbol{x}_{p}$, e o vetor de coeficientes $\boldsymbol{\beta}=\left(\beta_{1}, \beta_{2}, \ldots, \beta_{p}\right)^{\prime}$, $\epsilon \sim \mathrm{N}\left(\mathbf{0}, \sigma^{2} \boldsymbol{I}\right)$. Executa-se a centralização das variáveis, da maneira apresentada no Capítulo anterior.

A seguir propõe-se o uso de um modelo hierárquico, composto por três elementos:

1. Uma probabilidade $P(\mathcal{M})$ para cada possível modelo $\mathcal{M}$.

2. Uma distribuição a priori, $P\left(\theta_{\mathcal{M}} \mid \mathcal{M}\right)$ para o parâmetro $\theta_{\mathcal{M}}$ associado ao modelo $\mathcal{M}$.

3. Uma probabilidade associada às observações, condicional aos parâmetros, $P\left(Y \mid \mathcal{M}, \theta_{\mathcal{M}}\right)$.

Como o objetivo do método é encontrar o melhor modelo, a probabilidade a posteriori relevante é $P(\mathcal{M} \mid Y)$, ou seja, a probabilidade de determinado modelo ser o modelo real, dada a informação contida nos dados.

Pelo Teorema de Bayes: 
Primeiro:

$$
P(\mathcal{M} \mid Y)=\frac{P(Y \mid \mathcal{M}) P(\mathcal{M})}{\sum_{\mathcal{M}^{\prime}} P\left(Y \mid \mathcal{M}^{\prime}\right) P\left(\mathcal{M}^{\prime}\right)},
$$

e como o número de possíveis modelos é finito, o denominador é uma somatória de probabilidades sobre todos os possíveis modelos $\mathcal{M}^{\prime}$.

Por outro lado, a probabilidade $P(Y \mid \mathcal{M})$ é:

$$
P(Y \mid \mathcal{M})=\int P\left(Y \mid \mathcal{M}, \theta_{\mathcal{M}}\right) P\left(\theta_{\mathcal{M}} \mid \mathcal{M}\right) d \theta_{\mathcal{M}}
$$

Substituindo essa expressão em $P(\mathcal{M} \mid Y)$ obtém-se:

$$
P(\mathcal{M} \mid Y)=\frac{P(\mathcal{M}) \int P\left(Y \mid \mathcal{M}, \theta_{\mathcal{M}}\right) P\left(\theta_{\mathcal{M}} \mid \mathcal{M}\right) d \theta_{\mathcal{M}}}{\sum_{\mathcal{M}^{\prime}} P\left(\mathcal{M}^{\prime}\right) \int P\left(Y \mid \mathcal{M}^{\prime}, \theta_{\mathcal{M}^{\prime}}\right) P\left(\theta_{\mathcal{M}^{\prime}} \mid \mathcal{M}^{\prime}\right) d \theta_{\mathcal{M}^{\prime}}}
$$

\subsubsection{O modelo}

Será descrita a seguir a forma específica dos elementos do modelo proposto pelos autores.

Cada modelo será representado com um vetor de componentes binários $\gamma=\left(\gamma_{1}, \ldots, \gamma_{p}\right)^{\prime}$ onde $\gamma_{i}$ assume os valores 0 ou 1 , que representam se a $i$-ésima variável explicativa está inclusa no modelo ou não. Além disso, $\boldsymbol{\beta}_{\boldsymbol{\gamma}}$ representa o vetor de coeficientes das variáveis explicativas inclusas (ou seja, não nulas) no modelo $\boldsymbol{\gamma}$, a dimensão de $\boldsymbol{\beta}_{\gamma}$ é igual a $|\boldsymbol{\gamma}|=\sum \gamma_{i}$.

Com essa notação, podemos identificar os três elementos do modelo hierárquico descrito anteriormente.

1. $\boldsymbol{Y} \mid \boldsymbol{\gamma}, \boldsymbol{\beta} \sim N\left(\boldsymbol{X}_{\boldsymbol{\gamma}} \boldsymbol{\beta}_{\gamma}, \sigma^{2} \boldsymbol{I}_{(n)}\right)$,

no qual $\boldsymbol{X}_{\gamma}$ representa a matriz de planejamento contendo apenas as colunas das variáveis explicativas do modelo $\gamma$

2. $\beta_{i} \mid \gamma_{i} \sim\left(1-\gamma_{i}\right) 0+\gamma_{i} \frac{\tau \exp \left(-\tau\left|\beta_{i}\right|\right)}{2}$,

ou seja, caso a variável seja incluída no modelo, o correspondente parâmetro segue uma distribuição de Laplace $(0, \tau)$.

3. $P(\gamma) \propto q^{|\gamma|}(1-q)^{p-|\gamma|} \sqrt{\operatorname{det}\left(\boldsymbol{X}_{\gamma}^{\prime} \boldsymbol{X}_{\gamma}\right)}$

ou seja, a distribuição a priori de $\boldsymbol{\gamma}$ é similar a uma distribuição binomial, porém utiliza o determinante de $\boldsymbol{X}_{\gamma}^{\prime} \boldsymbol{X}_{\gamma}$ como peso. Isso é feito para diminuir a probabilidade do modelo selecionado ter variáveis explicativas muito correlacionadas, pois a dependência linear entre as colunas da matriz de planejamento faz com que esse determinante se aproxime de zero.

Note-se também que a multiplicação dos três termos é igual à distribuição conjunta $P\left(\boldsymbol{\gamma}, \boldsymbol{\beta}_{\gamma}, \boldsymbol{Y}\right)$, pois:

$$
P(\boldsymbol{Y} \mid \boldsymbol{\gamma}, \boldsymbol{\beta}) . P(\boldsymbol{\beta} \mid \boldsymbol{\gamma}) \cdot P(\boldsymbol{\gamma})=\frac{P\left(\boldsymbol{\gamma}, \boldsymbol{\beta}_{\boldsymbol{\gamma}}, \boldsymbol{Y}\right)}{P\left(\boldsymbol{\gamma}, \boldsymbol{\beta}_{\gamma}\right)} \cdot \frac{P\left(\boldsymbol{\gamma}, \boldsymbol{\beta}_{\gamma}\right)}{P(\boldsymbol{\gamma})} \cdot P(\boldsymbol{\gamma})=P\left(\boldsymbol{\gamma}, \boldsymbol{\beta}_{\boldsymbol{\gamma}}, \boldsymbol{Y}\right)
$$


Aplicando o resultado (4.1) na estrutura específica apresentada anteriormente obtém-se a distribuição à posteriori:

$$
\begin{aligned}
P(\mathcal{M} \mid \boldsymbol{Y})=P(\gamma \mid \boldsymbol{Y}) \propto q^{|\gamma|}(1-q)^{p-|\gamma|} \sqrt{\operatorname{det}\left(\boldsymbol{X}_{\gamma}^{\prime} \boldsymbol{X}_{\gamma}\right)}\left(\frac{\tau}{2}\right)^{|\gamma|}\left(\frac{1}{\sqrt{2 \pi \sigma^{2}}}\right)^{n} \times \\
\times \exp \left(\frac{\left\|\boldsymbol{Y}-\boldsymbol{X}_{\gamma} \boldsymbol{\beta}_{\gamma}\right\|^{2}-2 \sigma^{2} \tau \sum_{i \in \gamma}\left|\beta_{i}\right|}{2 \sigma^{2}}\right)
\end{aligned}
$$

Pode-se resumir a expressão anterior por:

$$
P(\boldsymbol{\gamma} \mid \boldsymbol{Y})=C(\boldsymbol{Y}) w^{|\gamma|} \times \int_{-\infty}^{\infty} \ldots \int_{-\infty}^{\infty} \frac{\sqrt{\operatorname{det}\left(\boldsymbol{X}_{\gamma}^{\prime} \boldsymbol{X}_{\gamma}\right)}}{\left(\sqrt{2 \pi \sigma^{2}}\right)^{|\gamma|}} \times \exp \left(-\frac{\left\|\boldsymbol{Y}-\boldsymbol{X}_{\gamma} \boldsymbol{\beta}_{\gamma}\right\|^{2}+\lambda \sum_{i \in \gamma}\left|\beta_{i}\right|}{2 \sigma^{2}}\right) d \boldsymbol{\beta}_{\gamma}
$$

com:

$$
\begin{gathered}
w=\left(\frac{q}{1-q} \frac{\tau}{2} \sqrt{2 \pi \sigma^{2}}\right), \\
\lambda=2 \sigma^{2} \tau
\end{gathered}
$$

$C(\boldsymbol{Y})$ uma constante contendo todos os termos que não dependem de $\boldsymbol{\gamma}$. Note-se que o termo $\left(\sqrt{2 \pi \sigma^{2}}\right)^{|\gamma|}$ foi incluído na equação e é redundante (aparece multiplicando no numerador e denominador), porém a inclusão desse termo é necessária para fazer a aproximação de Laplace, que será utilizada à frente.

O modelo escolhido por este método é aquele que apresentar a maior probabilidade à posteriori $P(\mathcal{M} \mid \boldsymbol{Y})$. Porém, a integral em (4.3) não tem solução analítica. Os autores propõe focar a análise nos modelos cuja probabilidade pode ser estimada com maior precisão. Para isso, propõe a divisão de $\boldsymbol{\beta}_{\gamma}$ em dois termos, definindo:

$$
\beta_{\gamma}=\beta_{\gamma}^{*}+u
$$

Sendo:

$$
\boldsymbol{\beta}_{\gamma}^{*} \underset{\boldsymbol{\beta}_{\gamma}}{\operatorname{argmin}}\left(\left\|\boldsymbol{Y}-\boldsymbol{X}_{\gamma} \boldsymbol{\beta}_{\gamma}\right\|^{2}+\lambda \sum_{i \in \gamma}\left|\beta_{i}\right|\right)
$$

De acordo com (YUAN; JOSEPH; LIN, 2007, apêndice), é possível re-escrever (4.3) como:

$$
\begin{array}{r}
P(\gamma \mid \boldsymbol{Y})=C(\boldsymbol{Y}) w^{|\gamma|} \times \int_{-\infty}^{\infty} \ldots \int_{-\infty}^{\infty} \frac{\sqrt{\operatorname{det}\left(\boldsymbol{X}_{\gamma}^{\prime} \boldsymbol{X}_{\gamma}\right)}}{\left(\sqrt{2 \pi \sigma^{2}}\right)^{|\gamma|}} \times \\
\times \exp \left(-\frac{\left\|\boldsymbol{X}_{\gamma} \boldsymbol{u}\right\|^{2}-2 \tilde{\boldsymbol{Y}}_{\gamma}^{\prime} \boldsymbol{X}_{\gamma} \boldsymbol{u}+\lambda \sum_{i \in \boldsymbol{\gamma}}\left(\left|\beta_{i}^{*}+u_{i}\right|-\left|\beta_{i}^{*}\right|\right)}{2 \sigma^{2}}\right) d \boldsymbol{u} \times \\
\times \exp \left(-\frac{\min _{\boldsymbol{\beta}}\left(\left|\boldsymbol{Y}-\boldsymbol{X}_{\gamma} \boldsymbol{\beta}_{\gamma} \|^{2}+\lambda \sum_{i \in \gamma}\right| \beta_{i} \mid\right)}{2 \sigma^{2}}\right)
\end{array}
$$




$$
\tilde{Y}_{\gamma}=\boldsymbol{Y}-\boldsymbol{X}_{\gamma} \boldsymbol{\beta}_{\gamma}
$$

Será utilizada a aproximação de Laplace para o termo:

$$
\int_{-\infty}^{\infty} \ldots \int_{-\infty}^{\infty} \frac{\sqrt{\operatorname{det}\left(\boldsymbol{X}_{\gamma}^{\prime} \boldsymbol{X}_{\gamma}\right)}}{\left(\sqrt{2 \pi \sigma^{2}}\right)^{|\gamma|}} \times \exp \left(-\frac{\left\|\boldsymbol{X}_{\gamma} \boldsymbol{u}\right\|^{2}-2 \tilde{\boldsymbol{Y}}_{\gamma}^{\prime} \boldsymbol{X}_{\gamma} \boldsymbol{u}+\lambda \sum_{i \in \gamma}\left(\left|\beta_{i}^{*}+u_{i}\right|-\left|\beta_{i}^{*}\right|\right)}{2 \sigma^{2}}\right) d \boldsymbol{u}
$$

A aproximação de Laplace é derivada da expansão de Taylor e é definida da seguinte maneira (ERDÉLYI, 1956):

Seja $f(\boldsymbol{x})$ uma função multivariada com uma matriz Hessiana definida negativa, com $\boldsymbol{x}_{\mathbf{0}}$ tal que $f\left(\boldsymbol{x}_{\mathbf{0}}\right)=\max f(\boldsymbol{x})$.

Nestas condições:

$$
\lim _{n \rightarrow+\infty} \frac{\int_{a}^{b} e^{n f(\boldsymbol{x})}}{e^{n f\left(\boldsymbol{x}_{\mathbf{0}}\right)} \sqrt{\frac{2 \pi}{n}}^{d} \frac{1}{\sqrt{\left|-H(f)\left(\boldsymbol{x}_{\mathbf{0}}\right)\right|}}} d \boldsymbol{x}=1
$$

com $\mathrm{H}(\mathrm{f})$ representando a matriz Hessiana e $|\ldots|$ o determinante da matriz.

Para aplicar a aproximação define-se a função:

$$
f(\boldsymbol{u}) \equiv \frac{\left.\left\|\boldsymbol{X}_{\gamma} \boldsymbol{u}\right\|\right|^{2}-2 \tilde{\boldsymbol{Y}}_{\gamma} \boldsymbol{X}_{\gamma} \boldsymbol{u}+\lambda \sum_{i \in \gamma}\left(\left|\beta_{i}^{*}+u_{i}\right|-\left|\beta_{i}^{*}\right|\right)}{\sigma^{2}},
$$

para um vetor $p$ dimensional verifica-se que tal função é minimizada no ponto $\boldsymbol{u}^{*}=\mathbf{0}$.

Caso o modelo $\gamma$ não contenha nenhum $\beta_{i}=0$, então $f(\boldsymbol{u})$ é duplamente diferenciável (os autores denominam tal modelo como "regular"). Caso contrário, a função não será diferenciável no ponto $\boldsymbol{u}^{*}$ e tal modelo é denominado "irregular". Lembrando que $\beta_{i}=0$ com $i \in \boldsymbol{\gamma}$ significa que o modelo aceita a variável $i$ (ou seja $\gamma_{i}=1$ ), mas seu coeficiente é zero. Essa situação não é desejável em um modelo de regressão. Os autores provam que, dada a construção teórica do modelo, se $w \leq 1$ então a probabilidade de um modelo irregular sempre será menor do que a de um modelo regular que simplesmente exclui a variável cujo coeficiente é zero (YUAN; LIN, 2005, p. 1248). Como a análise será concentrada no caso específico em que $w=1$, o caso dos modelos irregulares não será estudado em mais detalhes.

Caso o modelo seja regular, verifica-se que:

$$
\left.\frac{\partial^{2} f(\boldsymbol{u})}{\partial \boldsymbol{u} \partial \boldsymbol{u}^{\prime}}\right|_{\boldsymbol{u}=\boldsymbol{u}^{*}}=\frac{1}{\sigma^{2}} \boldsymbol{X}_{\gamma}^{\prime} \boldsymbol{X}_{\gamma}
$$

Utilizando a aproximação de Laplace em (4.6) obtém-se:

$$
\int_{-\infty}^{\infty} \ldots \int_{-\infty}^{\infty} \frac{\sqrt{\operatorname{det}\left(\boldsymbol{X}_{\gamma}^{\prime} \boldsymbol{X}_{\gamma}\right)}}{\left(\sqrt{2 \pi \sigma^{2}}\right)^{|\gamma|}} \times \exp \left(-\frac{\left\|\boldsymbol{X}_{\gamma} \boldsymbol{u}\right\|^{2}-2 \tilde{\boldsymbol{Y}}_{\gamma}^{\prime} \boldsymbol{X}_{\gamma} \boldsymbol{u}+\lambda \sum_{i \in \gamma}\left(\left|\beta_{i}^{*}+u_{i}\right|-\left|\beta_{i}^{*}\right|\right)}{2 \sigma^{2}}\right) d \boldsymbol{u} \approx 1
$$


Com essa aproximação, é possível reescrever 4.5 como:

$$
P(\boldsymbol{\gamma} \mid \boldsymbol{Y}) \approx C(\boldsymbol{Y}) w^{|\gamma|} \times \exp \left(-\frac{\min _{\boldsymbol{\beta}_{\gamma}}\left(\left|\boldsymbol{Y}-\boldsymbol{X}_{\gamma} \boldsymbol{\beta}_{\gamma} \|^{2}+\lambda \sum_{i \in \gamma}\right| \beta_{i} \mid\right)}{2 \sigma^{2}}\right) .
$$

Pode-se notar que, caso $w$ seja fixado em 1, a expressão (4.7) é maximizada com a minimização do termo $h(\boldsymbol{\gamma})=\left(\min _{\boldsymbol{\beta}_{\boldsymbol{\gamma}}}\left(\left\|\boldsymbol{Y}-\boldsymbol{X}_{\gamma} \boldsymbol{\beta}_{\gamma}\right\|^{2}+\lambda \sum_{i \in \boldsymbol{\gamma}}\left|\beta_{i}\right|\right)\right)$. A partir disso, os autores apresentam a proposição:

Seja $\hat{\boldsymbol{\beta}}=\operatorname{argmin}_{\beta}\left(\left\|\boldsymbol{Y}-\boldsymbol{X}_{\boldsymbol{\gamma}} \boldsymbol{\beta}_{\boldsymbol{\gamma}}\right\|^{2}+\lambda \sum_{i=1}^{p}\left|\beta_{i}\right|\right)$ e seja $\hat{\boldsymbol{\gamma}}$ associado ao modelo regular que inclui todos os elementos não nulos de $\hat{\boldsymbol{\beta}}$ em $\hat{\boldsymbol{\gamma}}$. Então $\hat{\boldsymbol{\gamma}}$ será o modelo que minimiza $h(\boldsymbol{\gamma})$, e , portanto, é o modelo com a maior probabilidade à posteriori.

É possível provar a proposição notando que $h$ é decrescente em $\gamma$, no sentido que, se $\gamma_{1}$ é um submodelo de $\boldsymbol{\gamma}_{\mathbf{2}}{ }^{1}$, então $h\left(\boldsymbol{\gamma}_{\mathbf{1}}\right) \geq h\left(\boldsymbol{\gamma}_{\mathbf{2}}\right)$, e ao mesmo tempo o modelo é regular, ou seja, excluí os $\beta_{i}$ iguais a zero.

Assim, como consequência dessa preposição, o modelo $\hat{\gamma}$ é o escolhido pelo método proposto por (YUAN; JOSEPH; LIN, 2007)

Além disso, nota-se que o modelo final encontrado corresponde exatamente àquele que seria obtido pelo procedimento tradicional do LASSO.

\subsubsection{O uso do método de Bayes empírico para a estimação do LASSO}

Assim como na formulação original do LASSO, deve-se estabelecer um valor para $\lambda \mathrm{e}$ também para $\sigma^{2}$. Note-se que o parâmetro $\tau$ da distribuição à priori também depende dessas duas quantidades.

A diferença do método proposto pelos autores e o LASSO tradicional é que, devido à sua formulação bayesiana, é possível utilizar técnicas do método de Bayes empírico para definir os parâmetros do procedimento LASSO.

O procedimento de Bayes empírico é uma metodologia que busca, ao contrário da inferência bayesiana tradicional, estimar os parâmetros da distribuição à priori (que, nesse caso, correspondem aos parâmetros do LASSO). Isso é feito a partir de uma modificação da metodologia da verossimilhança condicional proposta por George e Foster (2000), utilizando a distribuição $f\left(\boldsymbol{Y} \mid \boldsymbol{\gamma}, \sigma^{2}, \lambda\right)$ :

$$
\begin{gathered}
f\left(\boldsymbol{Y} \mid \boldsymbol{\gamma}, \sigma^{2}, \lambda\right)=\int_{-\infty}^{\infty} \ldots \int_{-\infty}^{\infty} P(\boldsymbol{Y} \mid \boldsymbol{\gamma}, \boldsymbol{\beta}) \times P(\boldsymbol{\beta} \mid \boldsymbol{\gamma}) d \boldsymbol{\beta}_{\boldsymbol{\gamma}}= \\
=\int_{-\infty}^{\infty} \ldots \int_{-\infty}^{\infty} \frac{\exp \left(-\frac{1}{\sigma^{2}}\left(\left\|\boldsymbol{Y}-\boldsymbol{X}_{\gamma} \boldsymbol{\beta}_{\gamma}\right\|^{2}\right)\right)}{\left(\sqrt{2 \pi \sigma^{2}}\right)^{n}} \times\left(\frac{\tau}{2}\right)^{|\gamma|} \exp \left(\sum_{i \in \boldsymbol{\gamma}}-\tau\left|\beta_{i}\right|\right) d \boldsymbol{\beta}_{\boldsymbol{\gamma}}
\end{gathered}
$$

Pode-se substituir $\tau=\frac{\lambda}{2 \sigma^{2}}$ e reorganizar os termos de maneira a obter:

$\overline{1} \gamma_{2}$ contém as mesmas variáveis explicativas que $\gamma_{1}$ e variáveis explicativas adicionais. 
$f\left(\boldsymbol{Y} \mid \boldsymbol{\gamma}, \lambda, \sigma^{2}\right)=\int_{-\infty}^{\infty} \ldots \int_{-\infty}^{\infty}\left(\frac{1}{2 \sigma^{2}}\right)^{n} \exp \left(-\frac{\| \boldsymbol{Y}-\left.\boldsymbol{X}_{\boldsymbol{\gamma}} \boldsymbol{\beta}_{\boldsymbol{\gamma}}\right|^{2}}{2 \sigma^{2}}\right) \times\left(\frac{\lambda}{4 \sigma^{2}}\right)^{|\gamma|} \exp \left(-\frac{\lambda \sum_{i \in \boldsymbol{\gamma}}\left|\beta_{i}\right|}{2 \sigma^{2}}\right) d \boldsymbol{\beta}_{\boldsymbol{\gamma}}$

Os autores notam que essa a abordagem não é exatamente igual à de George e Foster (2000) pois naquele caso, a verossimilhança derivada é obtida por $P(\gamma \mid q) \times P\left(\boldsymbol{Y} \mid \boldsymbol{\gamma}, \sigma^{2}\right)$, e, portanto, a função obtida anteriormente não é uma verossimilhança, mas decidem manter o nome de verossimilhança condicional devido à similaridade entre as duas abordagens.

Para cada valor de $\lambda$, existe um modelo $\hat{\gamma}_{\lambda}$ que será o modelo escolhido para aquele valor de $\lambda$. A seleção de $\lambda$ é feita maximizando $f\left(\boldsymbol{Y} \mid \hat{\gamma_{\lambda}}, \lambda, \sigma^{2}\right)$. Como são levados em conta os modelos regulares, a expressão 4.8 pode ser aproximada pela mesma aproximação de Laplace utilizada em (4.7) fornecendo:

$f\left(\boldsymbol{Y} \mid \hat{\gamma_{\lambda}}, \lambda, \sigma^{2}\right) \approx\left(\frac{1}{\sqrt{2 \pi \sigma^{2}}}\right)^{n-\left|\hat{\gamma_{\lambda}}\right|}\left(\frac{\lambda}{4 \sigma^{2}}\right)^{\hat{\gamma_{\lambda}}}\left(\operatorname{det}\left(\boldsymbol{X}_{\hat{\gamma_{\lambda}}}^{\prime} \boldsymbol{X}_{\hat{\gamma_{\lambda}}}\right)\right)^{\frac{1}{2}} \times \exp \left(-\frac{\min _{\beta}\left(|| \boldsymbol{Y}-\left.\boldsymbol{X}_{\hat{\gamma_{\lambda}}} \boldsymbol{\beta}_{\hat{\gamma_{\lambda}}}\right|^{2}\right)+\lambda \sum_{i \in \hat{\gamma_{\lambda}}}\left|\beta_{i}\right|}{2 \sigma^{2}}\right)$,

o que é equivalente a minimizar:

$\operatorname{MVC}(\lambda) \equiv\left(n+\left|\hat{\gamma_{\lambda}}\right|\right)\left[\ln \left(\frac{\min _{\beta}\left(\left.|| \boldsymbol{Y} \boldsymbol{X}_{\hat{\gamma_{\lambda}}} \boldsymbol{\beta}_{\hat{\gamma_{\lambda}}}\right|^{2}\right)+\lambda \sum_{i \in \hat{\gamma_{\lambda}}}^{p}\left|\beta_{i}\right|}{n+\left|\hat{\gamma_{\lambda}}\right|}\right)+1\right]+\ln \left(\operatorname{det}\left(\boldsymbol{X}_{\hat{\gamma_{\lambda}}}^{\prime} \boldsymbol{X}_{\hat{\gamma_{\lambda}}}\right)\right)-2\left|\hat{\gamma_{\lambda}}\right| \ln \left(\frac{\sqrt{2 \pi} \lambda}{4}\right)$,

na qual $M V C$ é a máxima vareossimilhança condicional com o estimador para $\sigma^{2}$ :

$$
\hat{\sigma}^{2}=\min _{\boldsymbol{\beta}}\left(\left\|\boldsymbol{Y}-\boldsymbol{X}_{\hat{\gamma_{\lambda}}} \boldsymbol{\beta}_{\hat{\gamma_{\lambda}}}\right\|^{2}+\lambda \sum_{i \in \hat{\gamma_{\lambda}}}^{p}\left|\beta_{i}\right|\right) /\left(n+\left|\hat{\gamma}_{\lambda}\right|\right)
$$

Como é possível calcular $\hat{\gamma}_{\lambda}$ para cada valor de $\lambda$, determina-se $\operatorname{MVC}(\lambda)$ para cada $\lambda$, e assim é possível minimizar a expressão $M V C$. O valor de $\lambda$ obtido será utilizado para estimar $\sigma^{2}$ e com isso os autores efetuam a seleção de modelos pelo método LASSO utilizando o procedimento de Bayes empírico.

\subsection{Interpretação Hierárquica alternativa e Amostrador de Gibbs}

Park e Casella (2008a), bem como sua versão estendida², propõe outra interpretação para o uso da distribuição de Laplace como distribuição à priori para os parâmetros $\beta_{i}$. Para isso, eles propõe um modelo hierárquico com o vetor $\beta$ com distribuição Normal multivariada, e sua matriz de covariância dependente de variáveis aleatórias do tipo exponencial. Isso porque a distribuição de Laplace pode ser escrita como uma mistura de distribuições Normais com um parâmetro de mistura exponencial. Além disso, os autores propõe uma implementação do procedimento LASSO via amostrador de Gibbs.

2 (PARK; CASELLA, 2008b), disponível em:

<https://pdfs.semanticscholar.org/5b51/ce3bbe7791e1533be7d4d76b2452bf043954.pdf> 
Os autores notam que o procedimento em si não seleciona variáveis automaticamente, porém proporciona valores de desvio padrão e intervalos de credibilidade que podem ser utilizados para a seleção de modelos.

\subsubsection{Distribuição de Laplace como mistura de distribuições Normais}

Os autores notam que o LASSO pode ser interpretado como uma distribuição à posteriori bayesiana se o modelo for especificado como:

$$
\begin{aligned}
\boldsymbol{y} \mid \boldsymbol{\mu}, \boldsymbol{X}, \boldsymbol{\beta}, \boldsymbol{\sigma}^{2} & \sim N_{n}\left(\mu \mathbf{1}_{n}+\boldsymbol{X} \boldsymbol{\beta}, \sigma^{2} \boldsymbol{I}_{n}\right) \\
\boldsymbol{\beta} & \sim \prod_{j=1}^{p} \frac{\lambda}{2} e^{-\lambda\left|\beta_{j}\right|}
\end{aligned}
$$

com $n$ observações e $p$ variáveis explicativas. Os valores $\mu$ e $\sigma^{2}$ podem ser considerados tanto como constantes quanto variáveis aleatórias (no modelo dos autores, ambos são variáveis aleatórias). Com essa estrutura, as distribuições à posteriori de $\beta$ e $\sigma^{2}$ se tornam:

$$
P\left(\boldsymbol{\beta}, \sigma^{2} \mid \tilde{\boldsymbol{y}}\right) \propto P\left(\sigma^{2}\right)\left(\sigma^{2}\right)^{-\frac{n}{2}} \exp \left(-\frac{1}{2 \sigma^{2}}\left(\boldsymbol{y}-\mu \mathbf{1}_{n}-\boldsymbol{X} \boldsymbol{\beta}\right)^{\prime}\left(\boldsymbol{y}-\mu \mathbf{1}_{n}-\boldsymbol{X} \boldsymbol{\beta}\right)-\lambda \sum_{j=1}^{p}\left|\beta_{j}\right|\right)
$$

Pode-se simplificar o termo no exponencial, calculando-se a média sobre $y, \bar{y}$ e fazendo $\tilde{\boldsymbol{y}}=\boldsymbol{y}-\bar{y} \mathbf{1}_{n}$, substituindo e integrando em $\mu$ a posteriori será:

$$
P\left(\boldsymbol{\beta}, \sigma^{2} \mid \tilde{\boldsymbol{y}}\right) \propto P\left(\sigma^{2}\right)\left(\sigma^{2}\right)^{-\frac{(n-1)}{2}} \exp \left(-\frac{1}{2 \sigma^{2}}(\tilde{\boldsymbol{y}}-\boldsymbol{X} \boldsymbol{\beta})^{\prime}(\tilde{\boldsymbol{y}}-\boldsymbol{X} \boldsymbol{\beta})-\lambda \sum_{j=1}^{p}\left|\beta_{j}\right|\right),
$$

Essa substituição no termo do exponencial e a integração serão explicados em detalhes à frente. A maximização em $\boldsymbol{\beta}$ em (4.11) resulta no estimador LASSO usual.

Os autores se utilizam a seguinte representação da distribuição de Laplace, entendida como uma mistura de distribuições normais, com um parâmetro de mistura exponencial:

$$
\frac{a}{2} e^{-a|z|}=\int_{0}^{\infty} \frac{1}{\sqrt{2 \pi s}} e^{-\frac{z^{2}}{2 s}} \frac{a^{2}}{2} e^{-\frac{a^{2} s}{2}} d s, a>0
$$

Com base nessa categorização, é proposto o seguinte modelo hierárquico:

$$
\begin{gathered}
\boldsymbol{y} \mid \mu, \boldsymbol{X}, \boldsymbol{\beta}, \sigma^{2} \sim N_{n}\left(\mu \mathbf{1}_{n}+\boldsymbol{X} \boldsymbol{\beta}, \sigma^{2} \boldsymbol{I}_{n}\right) \\
\boldsymbol{\beta} \mid \tau_{1}^{2} \tau_{2}^{2}, \ldots \tau_{p}^{2}, \sigma^{2} \sim N_{p}\left(\mathbf{0}_{p}, \sigma^{2} \boldsymbol{D}_{\tau}\right), \boldsymbol{D}_{\tau}=\operatorname{diag}\left(\tau_{1}^{2}, \ldots \tau_{p}^{2}\right) \\
\tau_{1}^{2} \tau_{2}^{2}, \ldots \tau_{p}^{2} \sim \prod_{j=1}^{p} \frac{\lambda^{2}}{2} e^{-\frac{\lambda^{2} \tau_{j}^{2}}{2}} d \tau_{j}^{2}, \tau_{1}^{2} \tau_{2}^{2}, \ldots \tau_{p}^{2}>0 \\
\sigma^{2} \sim P\left(\sigma^{2}\right) d \sigma^{2}
\end{gathered}
$$


no qual $P\left(\sigma^{2}\right)$ pode ser um distribuição à priori imprópria do tipo $P\left(\sigma^{2}\right)=\frac{1}{\sigma^{2}}$ ou uma distribuição do tipo gama-inversa:

$$
P\left(\sigma^{2}\right)=\frac{\gamma^{a}}{\Gamma(a)}\left(\sigma^{2}\right)^{-a-1} \exp \left(-\frac{\gamma}{\sigma^{2}}\right), \sigma^{2}>0
$$

Dessa forma, aplicando (4.12), a distribuição $P\left(\boldsymbol{\beta} \mid \sigma^{2}\right)$ tem a forma:

$$
P\left(\boldsymbol{\beta} \mid \sigma^{2}\right)=\prod_{j=1}^{p} \frac{\lambda}{2 \sqrt{\sigma^{2}}} \exp \left(\frac{-\lambda\left|\beta_{j}\right|}{\sqrt{\sigma^{2}}}\right) .
$$

Assim, é possível justificar o uso da distribuição de Laplace como distribuição à priori para a implementação do método LASSO Bayesiano.

\subsubsection{Uso do amostrador de Gibbs}

Utilizando uma distribuição à priori gama-inversa ${ }^{3}$ para $\sigma^{2}$, e uma distribuição "flat" para $\mu$ pode-se obter a distribuição conjunta:

$$
\begin{gathered}
f\left(\boldsymbol{y} \mid \mu, \boldsymbol{\beta}, \sigma^{2}\right) P\left(\sigma^{2}\right) P(\mu) \prod_{j=1}^{p} P\left(\boldsymbol{\beta}_{j} \mid \tau_{j}^{2}, \sigma^{2}\right) P\left(\tau_{j}^{2}\right)= \\
=\frac{1}{\left(2 \pi \sigma^{2}\right)^{\frac{n}{2}}} e^{\frac{1}{2 \sigma^{2}}\left(\boldsymbol{y}-\mu-\mathbf{1}_{n}-\boldsymbol{X} \boldsymbol{\beta}\right)^{\prime}\left(\boldsymbol{y}-\mu-\mathbf{1}_{n}-\boldsymbol{X} \boldsymbol{\beta}\right)} \frac{\gamma^{\alpha}}{\Gamma(a)}\left(\sigma^{2}\right)^{-a-1} e^{\frac{-\gamma}{\sigma^{2}}} \prod_{j=1}^{p} \frac{1}{\left(2 \pi \sigma^{2} \tau_{j}^{2}\right)^{\frac{1}{2}}} e^{\frac{\beta_{j}^{2}}{2 \sigma^{2} \tau_{j}^{2}}} \frac{\lambda^{2}}{2} e^{-\frac{\lambda^{2} \tau_{j}^{2}}{2}}
\end{gathered}
$$

Calculando-se a média $\bar{y}$, é possível re-escrever o termo no primeiro exponencial:

$$
\left(\boldsymbol{y}-\mu \mathbf{1}_{n}-\boldsymbol{X} \boldsymbol{\beta}\right)^{\prime}\left(\boldsymbol{y}-\mu \mathbf{1}_{n}-\boldsymbol{X} \boldsymbol{\beta}\right)=\left(\bar{y} \mathbf{1}_{n}-\mu \mathbf{1}_{n}\right)^{\prime}\left(\bar{y} \mathbf{1}_{n}-\mu \mathbf{1}_{n}\right)+(\tilde{\boldsymbol{y}}-\boldsymbol{X} \boldsymbol{\beta})^{\prime}(\tilde{\boldsymbol{y}}-\boldsymbol{X} \boldsymbol{\beta})
$$

Pode-se provar essa igualdade, expandindo primeiro o lado esquerdo:

$$
\begin{gathered}
\left(\boldsymbol{y}-\mu \mathbf{1}_{n}-\boldsymbol{X} \boldsymbol{\beta}\right)^{\prime}\left(\boldsymbol{y}-\mu \mathbf{1}_{n}-\boldsymbol{X} \boldsymbol{\beta}\right)= \\
=\boldsymbol{y}^{\prime} \boldsymbol{y}-\boldsymbol{y}^{\prime} \boldsymbol{X} \boldsymbol{\beta}-\boldsymbol{y}^{\prime} \mu \mathbf{1}_{n}-\mathbf{1}_{n}^{\prime} \mu \boldsymbol{y}+\mu^{2} \mathbf{1}_{n}^{\prime} \mathbf{1}_{n}+\mu \mathbf{1}_{n}^{\prime} \boldsymbol{X} \boldsymbol{\beta}-\boldsymbol{\beta}^{\prime} \boldsymbol{X}^{\prime} \boldsymbol{y}+\boldsymbol{\beta}^{\prime} \boldsymbol{X}^{\prime} \mu \mathbf{1}_{n}+\boldsymbol{\beta}^{\prime} \boldsymbol{X}^{\prime} \boldsymbol{X} \boldsymbol{\beta} .
\end{gathered}
$$

Os termos $\mu \mathbf{1}_{n}^{\prime} \boldsymbol{X} \boldsymbol{\beta}$ e $\boldsymbol{\beta}^{\prime} \boldsymbol{X}^{\prime} \mu \mathbf{1}_{n}$ são iguais a zero, pois envolvem a multiplicação $\boldsymbol{X}^{\prime} \mathbf{1}_{n}$ (ou seu transposto), que é nula, pois as colunas de $\boldsymbol{X}$ são centralizadas. Assim, a expansão do lado esquerdo se torna:

$$
\boldsymbol{y}^{\prime} \boldsymbol{y}-2 \boldsymbol{\beta}^{\prime} \boldsymbol{X}^{\prime} \boldsymbol{y}-2 n \mu \bar{y}+n \mu^{2}+\boldsymbol{\beta}^{\prime} \boldsymbol{X}^{\prime} \boldsymbol{X} \boldsymbol{\beta}
$$

A seguir expande-se o termo:

3 Dada pela densidade: $f(x ; \alpha, \beta)=\frac{\beta^{\alpha}}{\Gamma(\alpha)} x^{-\alpha-1} \exp \left(-\frac{\beta}{x}\right)$. No caso, se utilizou $\alpha=a$ e $\beta=\gamma$ 


$$
\begin{gathered}
(\tilde{\boldsymbol{y}}-\boldsymbol{X} \boldsymbol{\beta})^{\prime}(\tilde{\boldsymbol{y}}-\boldsymbol{X} \boldsymbol{\beta})=\tilde{\boldsymbol{y}}^{\prime} \tilde{\boldsymbol{y}}-\tilde{\boldsymbol{y}}^{\prime} \boldsymbol{X} \boldsymbol{\beta}-\boldsymbol{\beta}^{\prime} \boldsymbol{X}^{\prime} \tilde{\boldsymbol{y}}+\boldsymbol{\beta}^{\prime} \boldsymbol{X}^{\prime} \boldsymbol{X} \boldsymbol{\beta}= \\
=\tilde{\boldsymbol{y}}^{\prime} \tilde{\boldsymbol{y}}-2 \boldsymbol{\beta}^{\prime} \boldsymbol{X}^{\prime} \tilde{\boldsymbol{y}}+\boldsymbol{\beta}^{\prime} \boldsymbol{X}^{\prime} \boldsymbol{X} \boldsymbol{\beta}=\left(\boldsymbol{y} 1-\bar{y} \mathbf{1}_{n}\right)^{\prime}\left(\boldsymbol{y}-\bar{y} \mathbf{1}_{n}\right)-2 \boldsymbol{\beta}^{\prime} \boldsymbol{X}^{\prime}\left(\boldsymbol{y}-\bar{y} \mathbf{1}_{n}\right)+\boldsymbol{\beta}^{\prime} \boldsymbol{X}^{\prime} \boldsymbol{X} \boldsymbol{\beta}= \\
=\boldsymbol{y}^{\prime} \boldsymbol{y}-\boldsymbol{y}^{\prime} \bar{y} \mathbf{1}_{n}-\mathbf{1}_{n}^{\prime} \bar{y} \boldsymbol{y}+\mathbf{1}_{n}^{\prime} \bar{y} \overline{\mathbf{1}_{n}}-2 \boldsymbol{\beta}^{\prime} \boldsymbol{X}^{\prime} \boldsymbol{y}-2 \boldsymbol{\beta}^{\prime} \boldsymbol{X}^{\prime} \boldsymbol{y}-2 \boldsymbol{\beta}^{\prime} \boldsymbol{X}^{\prime} \bar{y} \mathbf{1}_{n}+\boldsymbol{\beta}^{\prime} \boldsymbol{X}^{\prime} \boldsymbol{X} \boldsymbol{\beta} .
\end{gathered}
$$

Os termos $\boldsymbol{y} \bar{y} \mathbf{1}_{n}, \mathbf{1}_{n}^{\prime} \bar{y} \boldsymbol{y}$ e $\mathbf{1}_{n}^{\prime} \bar{y} \bar{y} \mathbf{1}_{n}$ são iguais a $n \bar{y}^{2}$. O termo $2 \boldsymbol{\beta}^{\prime} \boldsymbol{X}^{\prime} \bar{y} \mathbf{1}_{n}=0$, pois envolve o termo $\boldsymbol{X}^{\prime} \mathbf{1}_{n}=0$, já que as colunas de $\boldsymbol{X}$ foram centralizadas.

Expandindo o termo $\left(\bar{y} \mathbf{1}_{n}-\mu \mathbf{1}_{n}\right)^{\prime}\left(\bar{y} \mathbf{1}_{n}-\mu \mathbf{1}_{n}\right)$ obtém-se $n \bar{y}^{2}-2 n \bar{y} \mu+n \mu^{2}$. Assim, somando os dois termos obtém-se:

$$
\begin{gathered}
\boldsymbol{y}^{\prime} \boldsymbol{y}-2 \boldsymbol{\beta}^{\prime} \boldsymbol{X}^{\prime} \boldsymbol{y}+\boldsymbol{\beta}^{\prime} \boldsymbol{X}^{\prime} \boldsymbol{X} \boldsymbol{\beta}+n \bar{y}^{2}-n \bar{y}^{2}-2 \bar{y} \mu+n \mu^{2}= \\
=\boldsymbol{y}^{\prime} \boldsymbol{y}-2 \boldsymbol{\beta}^{\prime} \boldsymbol{X}^{\prime} \boldsymbol{y}+\boldsymbol{\beta}^{\prime} \boldsymbol{X}^{\prime} \boldsymbol{X} \boldsymbol{\beta}-2 \bar{y} \mu+n \mu^{2},
\end{gathered}
$$

que é igual ao termo (4.14), provando-se a igualdade.

Além disso, têm-se:

$$
\left(\bar{y} \mathbf{1}_{n}-\mu \mathbf{1}_{n}\right)^{\prime}\left(\bar{y} \mathbf{1}_{n}-\mu \mathbf{1}_{n}\right)+(\tilde{\boldsymbol{y}}-\boldsymbol{X} \boldsymbol{\beta})^{\prime}(\tilde{\boldsymbol{y}}-\boldsymbol{X} \boldsymbol{\beta})=n(\bar{y}-\mu)^{2}+(\tilde{\boldsymbol{y}}-\boldsymbol{X} \boldsymbol{\beta})^{\prime}(\tilde{\boldsymbol{y}}-\boldsymbol{X} \boldsymbol{\beta}) .
$$

Com isso, temos a seguinte distribuição conjunta:

$$
\begin{gathered}
P\left(\boldsymbol{y}, \mu, \boldsymbol{\beta}, \sigma^{2}\right)=\frac{1}{\left(2 \pi \sigma^{2}\right)^{\frac{n}{2}}} e^{\frac{1}{2 \sigma^{2}} n(\bar{y}-\mu)^{2}} e^{\frac{1}{2 \sigma^{2}}(\tilde{\boldsymbol{y}}-\boldsymbol{X} \boldsymbol{\beta})^{\prime}(\tilde{\boldsymbol{y}}-\boldsymbol{X} \boldsymbol{\beta})} \times \\
\times \frac{\gamma^{\alpha}}{\Gamma(a)}\left(\sigma^{2}\right)^{-a-1} e^{\frac{-\gamma}{\sigma^{2}}} \prod_{j=1}^{p} \frac{1}{\left(2 \pi \sigma^{2} \tau_{j}^{2}\right)^{\frac{1}{2}}} e^{\frac{\beta_{j}^{2}}{2 \sigma^{2} \tau_{j}^{2}}} \frac{\lambda^{2}}{2} e^{-\frac{\lambda^{2} \tau_{j}^{2}}{2}}
\end{gathered}
$$

Essa representação permite a constatação de que $\mu \sim N\left(\bar{y}, \frac{\sigma^{2}}{n}\right)$ como será mostrado a seguir.

Reescreveram-se os termos da primeira linha da função anterior:

$$
\int_{-\infty}^{\infty} \frac{1}{\left(2 \pi \sigma^{2}\right)^{\frac{1}{2}}} \exp \left(-\frac{n}{2 \sigma^{2}}(\bar{y}-\mu)^{2}\right) \frac{1}{\left(2 \pi \sigma^{2}\right)^{\left(\frac{(n-1)}{2}\right)}} \exp \left(-\frac{1}{2 \sigma^{2}}(\tilde{\boldsymbol{y}}-\boldsymbol{X} \boldsymbol{\beta})^{\prime}(\tilde{\boldsymbol{y}}-\boldsymbol{X} \boldsymbol{\beta})\right) d \mu
$$

Com isso, pode-se integrar em relação à $\mu$, obtendo-se a distribuição conjunta condicionada a $\mu$ (dividida por uma constante), que em geral não tem grande importância no processo de inferência e não será utilizado no amostrador de Gibbs.

A distribuição conjunta marginal a $\mu$ é: 
$P\left(\boldsymbol{y}, \boldsymbol{\beta}, \boldsymbol{\sigma}^{\mathbf{2}}\right) \propto \frac{1}{\left(\sigma^{2}\right)^{\frac{n-1}{2}}} e^{\left(-\frac{1}{2 \sigma^{2}}(\tilde{\boldsymbol{y}}-\boldsymbol{X} \boldsymbol{\beta})^{\prime}(\tilde{\boldsymbol{y}}-\boldsymbol{X} \boldsymbol{\beta})\right)}\left(\sigma^{2}\right)^{-a-1} \exp \left(-\frac{\gamma}{\sigma^{2}}\right) \prod_{j=1}^{p} \frac{1}{\left(\sigma^{2} \tau_{j}^{2}\right)^{\frac{1}{2}}} e^{\left(-\frac{\beta_{j}^{2}}{2 \sigma^{2} \tau_{j}^{2}}\right)} \frac{\lambda^{2}}{2} e^{\left(-\frac{\lambda^{2} \tau_{j}^{2}}{2}\right)}$

Além disso, aplica-se a distribuição gama-inversa proposta anteriormente na equação (4.11). Após cálculos, obtém-se a seguinte distribuição à posteriori:

$$
P\left(\boldsymbol{\beta}, \boldsymbol{\sigma}^{2} \mid \tilde{\boldsymbol{y}}\right) \propto\left(\sigma^{2}\right)^{\frac{(n+p-1)}{2-a-1}} \exp \left(-\frac{1}{\sigma^{2}}\left(\frac{(\tilde{\boldsymbol{y}}-\boldsymbol{X} \boldsymbol{\beta})^{\prime}(\tilde{\boldsymbol{y}}-\boldsymbol{X} \boldsymbol{\beta})}{2}+\gamma\right)-\frac{\lambda}{\sqrt{\sigma^{2}}} \sum_{j=1}^{p}\left|\beta_{j}\right|\right)
$$

Caso $a=0$ e $\gamma=0$, o resultado equivale a utilizar a priori imprópria $\frac{1}{\sigma^{2}}$.

Como constatado anteriormente, com essa formulação, é possível obter as estimativas do procedimento LASSO usual maximizando o vetor $\boldsymbol{\beta}$.

Os autores notam, porém, que esse procedimento não é usual na inferência bayesiana, pois corresponderia a utilizar a moda da distribuição de $\beta$. Uma abordagem mais natural, na visão dos autores, seria utilizar a mediana ou a média do vetor como o estimador, sendo que os autores dão preferência à mediana.

Uma vez obtidos os componentes do estimador de $\beta$, que são as medianas das distribuições a posteriori de $\beta_{i}$ e os correspondentes intervalos de predição para os parâmetros de $\boldsymbol{\beta}$, pode-se efetuar a seleção de variáveis. Se o intervalo de predição contiver o valor zero, a correspondente variável não será incluída no modelo.

Porém, o cálculo dessas medianas depende de integrais que não tem forma fechada, ou pelo menos, forma fechada simples. Para resolver esse problema, os autores propõe o uso do amostrador de Gibbs.

Uma vez obtidos os componentes do estimador de $\beta$, que são as medianas das distribuições a posteriori de $\beta_{i}$ e os correspondentes intervalos de predição para os parâmetros de $\boldsymbol{\beta}$, pode-se efetuar a seleção de variáveis. Se o intervalo de predição contiver o valor zero, a correspondente variável não será incluída no modelo.

O amostrador de Gibbs é um procedimento que permite simular uma amostra de uma distribuição de probabilidade a partir de suas distribuições condicionais. Com isso, pode-se calcular diretamente estatísticas como média, mediana, moda, etc. Segue um pequeno resumo do amostrador de Gibbs, tal como apresentado em Casella e George (1992).

No caso bivariado, com (X,Y), o amostrador de Gibbs permite gerar amostras da distriuição $f_{X}(x)$ à partir das distribuições $f_{X \mid Y}(x \mid y)$ e $f_{Y \mid X}(y \mid x)$. Isso é feito por meio de um processo iterativo, iniciado com um valor arbitrário $y_{0}$ e simulando um valor aleatório das seguintes distribuições a cada passo: 


$$
\begin{gathered}
X_{i} \sim f\left(x \mid Y_{i}=y_{i}\right) \\
Y_{i+1} \sim f\left(y \mid X_{i}=x_{i}\right)
\end{gathered}
$$

A cada passo, esse processo gera uma amostra da distribuição $X_{i}$ e $Y_{i+1}$, que geram uma sequência de $n$ elementos denominada "sequência de Gibbs". Pode-se provar que, quando $n \rightarrow+\infty$ a distribuição de $X_{k}$ converge para a distribuição de $f_{X}(x)$.

É possível provar ((CASELLA; GEORGE, 1992, p. 169-170)) que a transição de $X_{0}$ para $X_{1}$ depende de dos valores de $Y_{0}$ de maneira:

$$
f_{X_{1} \mid X_{0}}\left(x_{1} \mid x_{0}\right)=\int f_{X_{1}, Y_{1}}\left(x_{1} \mid y\right) f_{Y_{1} \mid X_{0}}\left(y \mid x_{0}\right) d y
$$

e, de fato, essa mesma estrutura se preserva para todos os casos $f_{X_{t} \mid X_{t-1}}\left(x_{t} \mid x_{t-1}\right)$

Com isso, é possível estender essa equação sucessivamente de modo a calcular $f\left(X_{2} \mid X_{0}\right)$, $f\left(X_{3} \mid X_{0}\right)$, etc e chegar na generalização:

$$
f_{X_{k} \mid X_{0}}\left(x \mid x_{0}\right)=\int f_{X_{k} \mid X_{k-1}}(x \mid t) f_{X_{k-1} \mid X_{0}}\left(t \mid x_{0}\right) d t
$$

Quando $k \rightarrow \infty, f_{X_{k} \mid X_{0}}\left(x \mid x_{0}\right) \rightarrow f_{X}(x)$

Ainda no caso bivariado, suponha-se que é necessário obter a distribuição conjunta $f_{X, Y}(x, y)$, sendo as distribuições marginais $f_{X \mid y}(x \mid y)$ e $f_{Y \mid X}(y \mid x)$. Por definição $f_{X, Y}(x, y)=$ $f_{Y \mid X}(y \mid x) \times f_{X}(x)$, então, de maneira a encontrar a distribuição conjunta é necessário primeiro encontrar a distribuição $f_{X}(x)$. Para isso têm-se:

$$
\begin{gathered}
f_{X}(x)=\int f_{X, Y}(x, y) d y \\
f_{X}(x)=\int f_{X \mid Y}(x \mid y) f_{Y}(y) d y \\
f_{X}(x)=\int f_{X \mid Y}(x \mid y) \int f_{Y \mid X}(y \mid t) f_{X}(t) d t d y \\
f_{X}(x)=\int\left[\int f_{(X \mid Y)}(x \mid y) f_{Y \mid X}(y \mid t) d y\right] f_{X}(t) d t \\
f_{X}(x)=\int\left[h(x, t) f_{X}(t) d t\right]
\end{gathered}
$$

Com $h(x, t)=\int f_{(X \mid Y)}(x \mid y) f_{Y \mid X}(y \mid t) d y$. Conforme $k \rightarrow \infty$, pode-se igualar os termos dessa integral à (4.17) e (4.18) obtendo:

$$
\begin{gathered}
f_{X_{k} \mid X_{0}}\left(x \mid x_{0}\right) \rightarrow f_{X} \\
f_{X_{k} \mid X_{k-1}}(x \mid t) \rightarrow h(x, t) .
\end{gathered}
$$

Com isso, pode-se utilizar o amostrador de Gibbs para gerar uma simulação da distribuição conjunta de interesse a partir da simulação de valores das condicionais e da sequencia de Gibbs. 
Para mais de duas variáveis, o método é análogo, com a diferença de que o processo iterativo terá mais etapas a cada iteração, como, por exemplo:

$$
\begin{aligned}
X_{k} & \sim f\left(x \mid Y_{k}=y_{k}, Z_{k}=z_{k}\right) \\
Y_{k+1} & \sim f\left(y \mid X_{k}=x_{k}, Z_{k}=z_{k}\right) \\
Z_{k+1} & \sim f\left(z \mid X_{k}=x_{k}, Y_{k}=y_{k}\right) .
\end{aligned}
$$

Nesse caso serão necessários valores iniciais para $Y$ e $Z, y_{0}, z_{0}$ ao invés de apenas um, como no caso bivariado.

Para aplicar o amostrador de Gibbs ao LASSO bayesiano, é necessário inicialmente calcular as distribuições condicionais completas. Como a distribuição condicional completa é igual à distribuição conjunta (4.15) dividida pela distribuição marginal, integrada na variável desejada, os autores encontram facilmente as distribuições condicionais completas (marginais apenas a $\mu$ ), a partir da expressão (4.16).

Primeiramente, obtém-se a distribuição condicional de $\boldsymbol{\beta}$. Os termos que envolvem $\boldsymbol{\beta}$ em (4.16) são os termos da exponencial:

$$
-\frac{1}{2 \sigma^{2}}(\tilde{\boldsymbol{y}} \tilde{\boldsymbol{\beta}}-\boldsymbol{X} \boldsymbol{\beta})^{\prime}(\tilde{\boldsymbol{y}} \tilde{\boldsymbol{\beta}}-\boldsymbol{X} \boldsymbol{\beta})-\frac{1}{2 \sigma^{2}} \boldsymbol{\beta}^{\prime} \boldsymbol{D}_{\tau}^{-1} \boldsymbol{\beta}=-\frac{1}{2 \sigma^{2}}\left(\boldsymbol{\beta}^{\prime}\left(\boldsymbol{X}^{\prime} \boldsymbol{X}+\boldsymbol{D}_{\tau}^{-1}\right) \boldsymbol{\beta}-2 \tilde{\boldsymbol{y}}^{\prime} \boldsymbol{X} \boldsymbol{\beta}+\tilde{\boldsymbol{y}}^{\prime} \tilde{\boldsymbol{y}}\right)
$$

pois $\tilde{\boldsymbol{y}}^{\prime} \boldsymbol{X} \boldsymbol{\beta}$ é escalar.

Seja $\boldsymbol{A}=\boldsymbol{X}^{\prime} \boldsymbol{X}+\boldsymbol{D}_{\tau}^{-1}$. Nestas condições:

$\boldsymbol{\beta}^{\prime} \boldsymbol{A} \boldsymbol{\beta}-2 \tilde{\boldsymbol{y}}^{\prime} \boldsymbol{X} \boldsymbol{\beta}+\boldsymbol{y}^{\prime} \boldsymbol{y}=\left(\boldsymbol{\beta}-\boldsymbol{A}^{-1} \boldsymbol{X}^{\prime} \tilde{\boldsymbol{y}}\right)^{\prime} \boldsymbol{A}\left(\boldsymbol{\beta}-\boldsymbol{A}^{-1} \boldsymbol{X}^{\prime} \tilde{\boldsymbol{y}}\right)+\tilde{\boldsymbol{y}}^{\prime}\left(\boldsymbol{I}_{n}-\boldsymbol{X} \boldsymbol{A}^{-1} \boldsymbol{X}^{\prime}\right) \tilde{\boldsymbol{y}}$

Isso pode ser provado expandindo-se o segundo termo:

$$
\begin{gathered}
\left(\boldsymbol{\beta}-\boldsymbol{A}^{-1} \boldsymbol{X}^{\prime} \tilde{\boldsymbol{y}}\right)^{\prime} \boldsymbol{A}\left(\boldsymbol{\beta}-\boldsymbol{A}^{-1} \boldsymbol{X}^{\prime} \tilde{\boldsymbol{y}}\right)+\tilde{\boldsymbol{y}}^{\prime}\left(\boldsymbol{I}_{n}-\boldsymbol{X} \boldsymbol{A}^{-1} \boldsymbol{X}^{\prime}\right) \tilde{\boldsymbol{y}}= \\
\boldsymbol{\beta}^{\prime} \boldsymbol{A}^{-1} \boldsymbol{\beta}+\boldsymbol{\beta}^{\prime} \boldsymbol{A} \boldsymbol{A}^{-1} \boldsymbol{X}^{\prime} \tilde{\boldsymbol{y}}^{\prime}+\tilde{\boldsymbol{y}}^{\prime} \boldsymbol{X}\left(\boldsymbol{A}^{-1}\right)^{\prime} \boldsymbol{A} \boldsymbol{\beta}+\tilde{\boldsymbol{y}}^{\prime} \boldsymbol{X}\left(\boldsymbol{A}^{-1}\right)^{\prime} \boldsymbol{A} \boldsymbol{A}^{-1} \boldsymbol{X}^{\prime} \tilde{\boldsymbol{y}}+\tilde{\boldsymbol{y}}^{\prime} \tilde{\boldsymbol{y}}-\tilde{\boldsymbol{y}}^{\prime} \boldsymbol{X} \boldsymbol{A}^{-1} \boldsymbol{X}^{\prime} \tilde{\boldsymbol{y}}
\end{gathered}
$$

Como $\boldsymbol{\beta}^{\prime} \boldsymbol{A} \boldsymbol{A}^{-1} \boldsymbol{X}^{\prime} \tilde{\boldsymbol{y}}^{\prime}+\tilde{\boldsymbol{y}} \boldsymbol{X}\left(\boldsymbol{A}^{-1}\right)^{\prime} \boldsymbol{A} \boldsymbol{\beta}=2 \tilde{\boldsymbol{y}}^{\prime} \boldsymbol{X} \boldsymbol{\beta}$ e $\tilde{\boldsymbol{y}}^{\prime} \boldsymbol{X}\left(\boldsymbol{A}^{-1}\right)^{\prime} \boldsymbol{A} \boldsymbol{A}^{-1} \boldsymbol{X}^{\prime} \tilde{\boldsymbol{y}}-\tilde{\boldsymbol{y}}^{\prime} \boldsymbol{X} \boldsymbol{A}^{-1} \boldsymbol{X}^{\prime} \tilde{\boldsymbol{y}}=0$, prova-se a igualdade.

Com isso, isolando os termos que dependem de $\boldsymbol{\beta}$, constata-se que a distribuição à posteriori dessa variável é Normal multivariada da forma:

$$
\boldsymbol{\beta} \mid \tilde{\boldsymbol{y}}, \boldsymbol{\sigma}^{\mathbf{2}}, \boldsymbol{\tau}^{\mathbf{2}} \sim N\left(\left(\boldsymbol{A}^{-1} \boldsymbol{X}^{\prime} \tilde{\boldsymbol{y}}\right), \sigma^{2} \boldsymbol{A}^{-1}\right)
$$

Os termos envolvendo $\sigma^{2}$ são:

$$
\left(\sigma^{2}\right)^{-\frac{(n-1)}{2}-\frac{p}{2}-a-1} \exp \left\{-\frac{1}{\sigma^{2}}\left[\frac{(\tilde{\boldsymbol{y}}-\boldsymbol{X} \boldsymbol{\beta})^{\prime}(\tilde{\boldsymbol{y}}-\boldsymbol{X} \boldsymbol{\beta})}{2}+\frac{\boldsymbol{\beta}^{\prime} \boldsymbol{D}_{\tau}^{-1} \boldsymbol{\beta}}{2}+\gamma\right]\right\}
$$


Com isso, constata-se que a distribuição à posteriori de $\sigma^{2}$ é proporcional a uma distribuição gama-inversa, com parâmetros $\alpha=\frac{(n-1)}{2}+\frac{p}{2}+a$ e $b=\frac{(\tilde{\boldsymbol{y}}-\boldsymbol{X} \boldsymbol{\beta})^{\prime}(\tilde{\boldsymbol{y}}-\boldsymbol{X} \boldsymbol{\beta})}{2}+\frac{\boldsymbol{\beta}^{\prime} \boldsymbol{D}_{\tau}^{-1} \boldsymbol{\beta}}{2}+\gamma$.

Finalmente, a distribuição à posteriori de $\tau_{j}^{2}$, para cada $j$ tem a forma:

$$
\left(\tau_{j}^{2}\right)^{-\frac{1}{2}} \exp \left(-\frac{1}{2}\left(\frac{\beta_{j}^{2} / \sigma^{2}}{\tau_{j}^{2}}+\lambda^{2} \tau_{j}^{2}\right)\right)
$$

É possível fazer uma substituição de variável pelo método do Jacobiano , fazendo:

$$
\tau_{j}^{2}=\frac{1}{\eta_{j}^{2}}
$$

Com isso, a nova variável têm uma densidade proporcional a:

$$
\begin{array}{r}
\left(\eta^{2}\right)^{-\frac{3}{2}} \exp \left(-\frac{1}{2}\left(\frac{\beta_{j}^{2}}{\sigma^{2}} \eta_{j}^{2}+\frac{\lambda^{2}}{\eta_{j}^{2}}\right)\right) \\
\propto\left(\eta_{j}^{2}\right)^{-\frac{3}{2}} \exp \left(-\frac{\beta_{j}^{2}\left(\eta_{j}^{2}-\sqrt{\frac{\lambda^{2} \sigma^{2}}{\beta^{2}}}\right)^{2}}{2 \sigma^{2} \eta_{j}^{2}}\right) .
\end{array}
$$

Isso sugere uma distribuição Gaussiana-inversa, cuja parametrização ét:

$$
f(x)=\sqrt{\frac{b}{2 \pi}} x^{-\frac{3}{2}} \exp \left(-\frac{b(x-a)^{2}}{2 a^{2} x}\right), x>0
$$

Com $a>0$ a média e $b>0$ o parâmetro de escala, sendo a variância $\left(\frac{a^{3}}{b}\right)$. Nesse caso têm-se $a=\sqrt{\frac{\lambda^{2} \sigma^{2}}{\beta_{j}^{2}}}$ e $b=\lambda^{2}$.

Assim, com as três distribuições condicionais completas, dadas pelas equações (4.19), (4.20) e (4.21), é possível implementar o Amostrador de Gibbs, com a atualização em blocos para $\boldsymbol{\beta}$ e $\boldsymbol{\tau}^{2}$, ou seja, é feita a atualização de todas as variáveis $\beta_{j}$ contidas no vetor $\boldsymbol{\beta}$ na mesma etapa, e o mesmo vale para todas as variáveis $\tau_{j}^{2}$ presentes no vetor $\tau_{j}^{2}$ de maneira que o amostrador de Gibbs é executado a partir da atualização iterativa de três blocos de variávies (como discutido anteriormente), $\boldsymbol{\beta}, \boldsymbol{\tau}_{j}^{2}$ e $\sigma^{2}$.

Mesmo com essa formulação ainda é necessário estabelecer valores para $\alpha, \gamma$ e $\lambda$. Os autores se concentram somente na escolha de $\lambda$, fazendo apenas algumas observações sobre $\alpha$ e $\gamma$ : utilizar $\alpha=0$ e $\gamma=0$ é possível e corresponde a uma priori degenerada do tipo $\frac{1}{\sigma^{2}}$ para a variância, além disso, para valores $a \geq 0, \gamma \geq 0$ e $\lambda \geq 0$ geralmente se obtém distribuições à posteriori unimodais, o que assegura a convergência do amostrador de Gibbs. Por outro lado, os autores também provam que utilizando-se diretamente a priori de Laplace (ou seja, sem a formulação hierárquica), não é possível assegurar unimodalidade.

Quanto à seleção do valor de $\lambda$, que equivale ao parâmetro do LASSO tradicional, os autores afirmam que é possível utilizar o processo de validação cruzada (como no LASSO usual). 
Alternativamente, pode-se selecionar $\lambda$ por meio de uma metodologia de Bayes empírico, com o auxílio do algoritmo EM conjuntamente com o Amostrador de Gibbs ou por meio da especificação de uma hiper-priori para $\lambda$. Neste caso no qual os autores sugerem o uso da priori:

$$
P\left(\lambda^{2}\right)=\frac{\delta^{r}}{\Gamma(r)}\left(\lambda^{2}\right)^{r-1} \exp \left(-\delta \lambda^{2}\right), \lambda>0, r>0 \text { e } \delta>0 \text {, ou seja, uma distribuição gama. }
$$

O algoritmo EM (Expectation - Maximization) funciona da seguinte maneira (ROBERT; CASELLA, 2004, p. 176 - 177):

Suponha existir uma amostra $X_{1}, X_{2}, \ldots, X_{n}$ variáveis aleatórias independentes, identicamente distribuídas, provenientes de uma distribuição $g(x \mid \theta)$ e que o objetivo seja calcular um estimador de máxima verossimilhança tal que $\hat{\theta}=\operatorname{argmax} L(\theta \mid \boldsymbol{x})=\prod_{i=1}^{n} g\left(x_{i} \mid \theta\right)$. Porém essa verossimilhança é difícil de calcular analiticamente, e como auxílio, os dados são aumentados por meio de uma nova variável $\boldsymbol{z}$ (chamada comumente de "variável latente"), com a distribuição conjunta $\boldsymbol{X}, \boldsymbol{Z} \sim f(\boldsymbol{x}, \boldsymbol{z} \mid \theta)$ conhecida e utiliza-se a identidade:

$$
k(\boldsymbol{z} \mid \theta, \boldsymbol{x})=\frac{f(\boldsymbol{x}, \boldsymbol{z} \mid \theta)}{g(\boldsymbol{x} \mid \theta)} \quad{ }^{5},
$$

para escrever a função de verossimilhança como:

$$
\log L(\theta \mid \boldsymbol{x})=\log L^{c}(\theta \mid \boldsymbol{x}, \boldsymbol{z})-\log k(\boldsymbol{z} \mid \theta, \boldsymbol{x}),
$$

na qual $L$ representa a função de verossimilhança de $\theta$ em relação a $\boldsymbol{x}$ e $L^{c}$ representa a função de verossimilhança "completa"(isto é, com os dados aumentados). A partir disso, pode-se calcular a esperança de todos os termos envolvidos com respeito a $k(\boldsymbol{z} \mid \theta, \boldsymbol{x})$ :

$$
\log L(\theta \mid \boldsymbol{x})=E_{\theta_{0}}\left(\log L^{c}(\theta \mid \boldsymbol{x}, \boldsymbol{z})\right)-E_{\theta_{0}}(\log k(\boldsymbol{z} \mid \theta, \boldsymbol{x})),
$$

lembrando que $E_{\theta_{0}}(\log L(\theta \mid \boldsymbol{x}))=\log L(\theta \mid \boldsymbol{x})$, por não envolver $\boldsymbol{z}$. Esse cálculo pode ser feito para qualquer valor $\theta_{0}$.

O objetivo do algoritmo é maximizar a verossimilhança $\log L(\theta \mid \boldsymbol{x})$, o que será feito por meio da maximização de (4.22), obtendo iterativamente valores do parâmetro $\theta$ mais próximos da estimativa de máxima verosimilhança(que não pode ser calculada diretamente).

Para maximizar a esperança da verossimilhança $\log L(\theta \mid \boldsymbol{x})$ basta maximizar $Q\left(\theta \mid \theta_{0}, \boldsymbol{x}\right)=$ $E_{\theta_{0}}\left(\log L^{c}(\theta \mid \boldsymbol{x}, \boldsymbol{z})\right)$. Essa maximização permite construir um processo iterativo da seguinte maneira, a partir de um valor inicial $\theta_{0}$ arbitrário da forma:

1. Estabelecer $\theta_{0}$

2. Computar $Q\left(\theta \mid \hat{\theta}_{m}, \boldsymbol{x}\right)=E_{\hat{\theta}_{m}}\left(\log L^{c}(\theta \mid \boldsymbol{x}, \boldsymbol{z})\right)$ (passo E)

3. Calcular $\theta_{m+1}=\underset{\theta}{\operatorname{argmax}} Q\left(\theta \mid \hat{\theta}_{m}, \boldsymbol{x}\right)($ Passo $\mathrm{M})$

4. Voltar ao passo 2 e repetir o procedimento até a convergência.

$\overline{5}$ sendo $k(\boldsymbol{z} \mid \theta, \boldsymbol{x})$ a densidade condicional de $k$ dados $\theta$ e $\boldsymbol{x}, f(\boldsymbol{x}, \boldsymbol{z} \mid \theta)$ a densidade condicional de $\boldsymbol{x}, \boldsymbol{z}$ dado $\theta$ e $g(\boldsymbol{x} \mid \theta)$ a densidade condicional de $\boldsymbol{x}$ dado $\theta$ 
Uma desvantagem do algoritmo EM é que ele garante convergência, mas não necessariamente ela ocorrerá no máximo global.

No caso em estudo, o parâmetro que será estimado é $\lambda$, as variáveis latentes são $\tau_{1} \ldots \tau_{p}$, e a densidade do modelo "completo"é aquela apresentada em (4.15). Os autores propõe o seguinte algoritmo para combinar o amostrador de Gibbs e o algoritmo EM (PARK; CASELLA, 2008b, p. 12):

a) Seja $\lambda^{(k)}$ o valor de $\lambda$ no passo $k$

b) Estabelece-se $k=0$ e escolhe-se $\lambda^{(0)}$

c) Gerar amostras da distribuição à posteriori de $\beta, \sigma^{2}, \boldsymbol{\tau}^{2}$ utilizando o amostrador de Gibbs descrito anteriormente, com $\lambda^{(k)}$.

d) Calcular uma aproximação a log-verossimilhança para $\lambda$ substituindo as esperanças de $\boldsymbol{\beta}, \sigma^{2}, \boldsymbol{\tau}^{2}$ pelas médias das amostras de Gibbs calculadas no passo anterior.(Passo E)

e) Calcular $\lambda^{k+1}$ como o valor de $\lambda$ que maximiza a esperança da log-verossimilhança calculada na etapa anterior (Passo M).

f) Voltar ao passo b) e repetir o procedimento até convergência.

A log-verossimilhança baseada na distribuição definida em (4.15) torna-se:

$$
\begin{aligned}
E_{\lambda(k)}\left(L\left(\lambda ; \boldsymbol{\tau}, \boldsymbol{y}, \boldsymbol{\beta}, \sigma^{2}\right)\right) & =-\left(\frac{(n+p-1)}{2}+a+1\right) \ln \left(\sigma^{2}\right)-\frac{1}{\sigma^{2}}\left(\frac{(\tilde{\boldsymbol{y}}-\boldsymbol{X} \boldsymbol{\beta})^{\prime}(\tilde{\boldsymbol{y}}-\boldsymbol{X} \boldsymbol{\beta})}{2}+\gamma\right)- \\
& -\frac{1}{2} \sum_{j=1}^{p} \ln \left(\tau_{j}^{2}\right)-\frac{1}{2} \sum_{j=1}^{p} \frac{\beta_{j}^{2}}{\sigma_{j}^{2} \tau_{j}^{2}}+p \ln \left(\lambda^{2}\right)-\frac{\lambda^{2}}{2} \sum_{j=1}^{p} \tau_{j}^{2}
\end{aligned}
$$

excluídas constantes aditivas não dependentes de $\lambda$. Na k-ésima iteração da etapa $\mathrm{E}$ do procedimento calcula-se a esperança dessa função com base em $\lambda^{(k)}$.

Os termos envolvendo $\lambda$, ou seja, aqueles que são relevantes para a maximização, encontram-se na seguinte função:

$$
Q\left(\lambda \mid \lambda^{k}\right)=p \ln \left(\lambda^{2}\right)-\frac{\lambda^{2}}{2} \sum_{p}^{j=1} E_{\lambda^{k}}\left(\tau_{j}^{2} \mid \tilde{\boldsymbol{y}}\right) .
$$

Após cálculos, verifica-se que a etapa da maximização permite a solução analítica:

$$
\lambda^{k+1}=\sqrt{\frac{2 p}{\sum_{j=1}^{p} E_{\lambda^{k}}\left(\tau_{j}^{2} \mid \tilde{\boldsymbol{y}}\right)}},
$$

lembrando que $E_{\lambda^{k}}\left(\tau_{j}^{2} \mid \tilde{\boldsymbol{y}}\right)$ é substituída pela média das amostras do amostrador de Gibbs calculadas com o valor de $\lambda$ anterior. 
Os autores sugerem que seja utilizado $\lambda^{0}=\frac{p \sqrt{\left(\hat{\sigma}^{2}\right)^{M Q O}}}{\sum_{j=1}^{p}\left|\left(\hat{\beta}_{j}^{2}\right)^{M Q O}\right|}$, na qual $\left(\hat{\sigma}^{2}\right)^{M Q O}$ e $\left(\hat{\beta}_{j}^{2}\right)^{M Q O}$ são as estimativas obtidas por mínimos quadrados ordinários no modelo inicial. Além disso, os autores notam que a combinação do amostrador de Gibbs e do algoritmo EM não leva exatamente a uma convergência, mas a uma flutuação (drift) em torno do estimador de máxima verossimilhança, com essa flutuação sendo reduzida se a cada iteração aumentarem-se o número de amostras de Gibbs.

Quanto à utilização de uma hiperpriori para $\lambda$, é importante notar que a distribuição segue uma gama em relação à $\lambda^{2}$ e não $\lambda$. Nesse caso, a distribuição conjunta em (4.15) é multiplicada por $P\left(\lambda^{2}\right)=\frac{\delta^{r}}{\Gamma(r)}\left(\lambda^{2}\right)^{r-1} \exp \left(-\delta \lambda^{2}\right)$. Assim, os termos envolvendo $\lambda$ na nova distribuição conjunta são:

$$
\left(\lambda^{2}\right)^{p+r-1} \exp \left(-\lambda^{2}\left(\frac{1}{2} \sum_{j=1}^{p} \tau_{j}^{2}+\delta\right)\right)
$$

Com isso, a distribuição à posteriori de $\lambda^{2}$ é uma distribuição gama com os parâmetros $\alpha=p+r$ e $\beta=\sum_{j=1}^{p} \frac{\tau_{j}^{2}}{2}+\delta$. Assim, basta incluir a simulação deste parâmetro no amostrador de Gibbs, agora com quatro blocos $\left(\boldsymbol{\beta}, \sigma^{2}, \boldsymbol{\tau}\right.$ e $\left.\lambda\right)$.

\subsection{Spike-and-Slab LASSO}

Ročková e George (2016b) propõe uma nova abordagem para a seleção de variáveis bayesiana por meio do uso de uma distribuição à priori do tipo "Spike-and-Slab", que é uma mistura de distribuições de Laplace. Na abordagem bayesiana, o procedimento LASSO pode ser encarado como uma solução para um problema de mínimos quadrados com um termo de penalização. O uso das prioris Spike-and-Slab possibilita duas vantagens importantes. Em primeiro lugar, elas permitem um termo de penalização que se adapta aos dados, o que é especialmente importante em situações que lidam com modelos esparsos, ou seja, que possuem inicialmente um grande número de variáveis explicativas porém a maior parte deles tem coeficiente zero. A segunda vantagem é que elas permitem a formulação de um algoritmo de seleção no qual o parâmetro de uma das duas distribuições Laplace (especificamente a "Spike") é sucessivamente alterado, de maneira a se tornar mais rígido a cada etapa. Esse algoritmo será explicado mais à frente.

Como nos modelos anteriores apresentados neste capítulo, seja um modelo dado por:

$$
\boldsymbol{Y}=\boldsymbol{X} \boldsymbol{\beta}+\epsilon,
$$

No qual $\boldsymbol{Y}$ é um vetor $n$ dimensional centrado e $\boldsymbol{X}_{n \times p}$ a matriz de planejamento com $p$ variáveis preditoras, e $\boldsymbol{\beta}=\left(\beta_{1}, \beta_{2}, \ldots, \beta_{p}\right)^{\prime}$ o vetor dos parâmetros associados às variáveis preditoras. Além disso $\epsilon \sim N_{p}\left(\mathbf{0}, \sigma^{2} I_{p}\right)$ com $\sigma^{2}=1$. Os regressores também serão centrados de maneira a satisfazer $\left\|\boldsymbol{X}_{j}\right\|^{2}=n$ para $1 \leq j \leq p$. O modelo foi idealizado para situações em que $p>n$ e a maioria dos elementos do vetor $\boldsymbol{\beta}$ são iguais a zero. O número de elementos não nulos será dado por $q$, tal que $q=\|\boldsymbol{\beta}\|_{0}$, ou seja, a dos elementos não nulos de $\boldsymbol{\beta}$ é igual a $q$. 
O estimador do vetor $\boldsymbol{\beta}$ via máxima verossimilhança penalizada terá a forma:

$$
\hat{\boldsymbol{\beta}}=\underset{\boldsymbol{\beta} \in R^{p}}{\operatorname{argmax}}\left(-\frac{1}{2}\|\boldsymbol{Y}-\boldsymbol{X} \boldsymbol{\beta}\|^{2}+\operatorname{pen}_{\lambda}(\boldsymbol{\beta})\right),
$$

na qual $\operatorname{pen}_{\lambda}(\boldsymbol{\beta})$ é a função de penalidade indexada pelo parâmetro $\lambda$. Essa penalidade pode ser separável, ou seja $\operatorname{pen}_{\lambda}(\boldsymbol{\beta})=\sum_{j=1}^{p} \rho_{\lambda}\left(\beta_{j}\right)$ (em que $\rho_{\lambda}\left(\beta_{j}\right)$ é a penalidade imposta a $\beta_{j}$ ), ou não-separável. A penalidade tradicional do LASSO, por exemplo, é separável, já que $\rho_{\lambda}\left(\beta_{j}\right)=$ $-\lambda\left|\beta_{j}\right|$. Esta penalidade tem a função de selecionar variáveis, mas também diminuir o vício do estimador obtido pelo procedimento LASSO. Os autores notam que qualquer estimador de máxima verossimilhança penalizada pode ser entendido como o estimador da moda de uma distribuição à posteriori obtida por meio de uma distribuição à priori $P(\boldsymbol{\beta} \mid \lambda)$, na qual pen ${ }_{\lambda}(\boldsymbol{\beta})=\ln (P(\boldsymbol{\beta} \mid \lambda))$ e, em particular, as penalidades separáveis surgem em casos nos quais as prioris para as componetes de $\beta$ são independentes.

Os autores propõe uma distribuição à priori do tipo Spike-and-Slab, que é obtida por meio de um modelo hierárquico, que também pode ser entendido como uma mistura de distribuições de Laplace com o parâmetro da mistura $\gamma$ sendo uma variável aleatória, ou seja:

$$
P(\boldsymbol{\beta} \mid \gamma)=\prod_{i=1}^{p}\left(\gamma_{i} \psi_{1}\left(\beta_{i}\right)+\left(1-\gamma_{i}\right) \psi_{0}\left(\beta_{i}\right)\right), \boldsymbol{\gamma} \sim P(\boldsymbol{\gamma})
$$

com,

$$
\begin{aligned}
& \psi_{1}(\beta)=\frac{\lambda_{1}}{2} \exp \left(-\lambda_{1}|\beta|\right) \\
& \psi_{0}(\beta)=\frac{\lambda_{0}}{2} \exp \left(-\lambda_{0}|\beta|\right)
\end{aligned}
$$

Observa-se que $\psi_{1}$ é a distribuição slab, ou difusa, que modela coeficientes não nulos e $\psi_{0}$ é a distribuição spike, ou concentrada, que modela os coeficientes próximos de zero. $\lambda_{1}$ é um valor "pequeno"(implicando em maior concentração da distribuição), enquanto $\lambda_{0}$ é um valor "grande", implicando mais dispersão. O vetor $\gamma=\left(\gamma_{1}, \ldots, \gamma_{p}\right)^{\prime}$, com $\gamma_{i} \in\{0,1\}$ indica os $2^{p}$ submodelos possíveis (esse vetor é similar ao vetor $\gamma$ do texto de Yuan e Lin (2005), discutido no início desse Capítulo).

Além disso, $P(\gamma)$ será dada por:

$$
P(\gamma \mid \theta)=\prod_{i=1}^{p} \theta^{\gamma_{i}}(1-\theta)^{1-\gamma_{i}}, \theta \sim P(\theta),
$$

tal que $\theta=P\left(\gamma_{i}=1 \mid \theta\right)$. Ou seja, o parâmetro $\theta$ descreve a probabilidade de cada preditor pertencer ao modelo final, e, por consequência, é não-nulo. Assim, o parâmetro $\theta$ está relacionado ao nível de esparsidade $q$.

Condicionando em $\theta$, a distribuição de $\boldsymbol{\beta}$ se torna:

$$
P(\boldsymbol{\beta} \mid \theta)=\prod_{i=1}^{p}\left(\theta \psi_{1}\left(\beta_{i}\right)+(1-\theta) \psi_{0}\left(\beta_{i}\right)\right)
$$


Com essa especificação, os autores voltam sua atenção para a função de penalidade $p e n_{\lambda}$, e, apesar de estudar um caso com uma função de penalidade separável, optam por utilizar uma penalidade não-separável. Argumentam que no caso separável a eficiência da seleção depende de uma pré-especificação de um valor $\theta$ próximo à fração de esparsidade $\frac{q}{p}$, enquanto que a penalidade não-separável é capaz de se adaptar ao nível de esparsidade por meio de uma distribuição à priori de $\theta, \theta \sim P(\theta)$, de maneira que:

$$
P(\boldsymbol{\beta})=\int_{0}^{1} \prod_{j=1}^{p}\left(\theta \psi_{1}\left(\beta_{j}\right)+(1-\theta) \psi_{0}\left(\beta_{j}\right)\right) d P(\theta),
$$

esta expressão pode ser re-escrita como:

$$
P(\boldsymbol{\beta})=\left(\frac{\lambda_{1}}{2}\right)^{p} \exp \left(-\lambda_{1}\|\boldsymbol{\beta}\|_{1}\right) \int_{0}^{1} \frac{\lambda^{p}}{\prod_{j=1}^{p} p_{\theta}^{\star}\left(\beta_{j}\right)} d P(\theta) .
$$

No qual $\|\boldsymbol{\beta}\|_{1}$ é a norma l-1 do vetor $\boldsymbol{\beta}\left(\sum_{j=1}^{n}\left|\beta_{j}\right|\right)$

$$
p_{\theta}^{\star}\left(\beta_{j}\right)=\frac{\theta \psi_{1}\left(\beta_{j}\right)}{\theta \psi_{1}\left(\beta_{j}\right)+(1-\theta) \psi_{0}\left(\beta_{j}\right)},
$$

além disso,

$$
p_{\theta}^{\star}\left(\beta_{j}\right)=P\left(\gamma_{j}=1 \mid \beta_{j}, \theta\right)=\left(1+\frac{\lambda_{0}}{\lambda_{1}} \frac{(1-\theta)}{\theta} \exp \left(-\left|\beta_{j}\right|\left(\lambda_{0}-\lambda_{1}\right)\right)\right)^{-1} .
$$

Com isso, os autores definem a função de penalidade:

$$
\operatorname{pen}_{N S}(\boldsymbol{\beta})=\ln \left(\frac{P(\boldsymbol{\beta})}{P\left(\mathbf{0}_{p}\right)}\right)=-\lambda_{1}|\boldsymbol{\beta}|+\ln \left(\frac{\int \frac{\theta^{p}}{\prod_{j=1}^{p} p_{\theta}^{\star}\left(\beta_{j}\right)} d P(\theta)}{\int \frac{\theta^{p}}{\prod_{j=1}^{p} p_{\theta}^{\star}(0)} d P(\theta)}\right)
$$

É possível provar (ROČKOVÁ; GEORGE, 2016b, p. 7, 11) que a derivada desta penalidade é:

$$
\frac{\partial p e n_{N S}(\boldsymbol{\beta})}{\partial\left|\beta_{j}\right|} \equiv-\lambda^{\star}\left(\beta_{j} ; \boldsymbol{\beta}_{\backslash j}\right)
$$

$\operatorname{Com} \lambda^{\star}\left(\beta_{j} ; \boldsymbol{\beta}_{\backslash j}\right)=p^{\star}\left(\beta_{j} ; \boldsymbol{\beta}_{\backslash j}\right) \lambda_{1}+\left(1-p^{\star}\left(\beta_{j} ; \boldsymbol{\beta}_{\backslash j}\right) \lambda_{0}\right.$,

e $p^{\star}\left(\beta_{j} ; \boldsymbol{\beta}_{\backslash j}\right) \equiv \int_{0}^{1} p_{\theta}^{\star}\left(\beta_{j}\right) P(\theta \mid \boldsymbol{\beta}) d \theta$.

Na qual $\boldsymbol{\beta}_{\backslash j}$ representa o vetor $\boldsymbol{\beta}$ com todos os seus elementos, exceto $\beta_{j}$.

É possível provar que, dada uma priori $P(\theta)$ :

$$
p^{\star}\left(\beta_{j} ; \boldsymbol{\beta}_{\backslash j}\right)=p_{\theta_{j}}^{\star}\left(\beta_{j}\right),
$$

$\operatorname{com} \theta_{j}=E\left(\theta \mid \boldsymbol{\beta}_{\backslash j}\right)$, ou seja, na expressão(4.24) substitui-se $\theta$ por sua esperança em relação ao vetor $\boldsymbol{\beta}_{\backslash j}$. 
Esse resultado é importante pois permite a implementação de um método de resolução para cada $\beta_{j}$ sucessivamente, mantendo fixo o vetor $\boldsymbol{\beta}_{\backslash j}$.

Com isso é possível re-escrever a penalidade não-separável proposta como uma penalidade separável, mas com a vantagem da adaptação ao nível de esparsidade discutida anteriormente, tal que:

$$
\tilde{\rho}\left(\beta_{j}, \boldsymbol{\beta}_{\backslash j}\right) \equiv \ln \frac{P\left(\beta_{j} ; \boldsymbol{\beta}_{\backslash j}\right)}{P\left(0 ; \boldsymbol{\beta}_{\backslash j}\right)}=-\lambda_{1}\left|\beta_{j}\right|+\ln \left(\frac{p^{\star}\left(0, \boldsymbol{\beta}_{\backslash j}\right)}{p^{\star}\left(\beta_{j}, \boldsymbol{\beta}_{\backslash j}\right)}\right),
$$

no qual $P\left(\beta_{j} ; \boldsymbol{\beta}_{\backslash j}\right)$ é outra maneira de escrever $P(\boldsymbol{\beta})$. Além disso,

$$
\tilde{\rho}\left(\beta_{j}, \boldsymbol{\beta}_{\backslash j}\right)=\rho\left(\beta_{j} \mid \theta_{j}\right), \operatorname{com} \quad \theta_{j}=E\left(\theta \mid \boldsymbol{\beta}_{\backslash j}\right) .
$$

$\rho\left(\beta_{j} \mid \theta\right)$ é usado para representar cada componente do somatório de uma penalidade separável, fazendo assim a ligação entre o caso separável e não-separável, em que a penalidade é separável, porém se adapta ao nível de esparcidade por meio de $E\left(\theta \mid \boldsymbol{\beta}_{\backslash j}\right)$.

A partir disso, os autores chegam ao seguinte estimador para a moda da distribuição à posteriori de $\boldsymbol{\beta}$ (à partir da máxima verossimilhança ponderada pela penalidade acima):

$$
\hat{\beta}_{j}=\left\{\begin{array}{l}
0, \mathrm{se}\left|z_{j}\right| \leq \Delta_{j}, \\
\frac{1}{n}\left[\left|z_{j}\right|-\lambda_{\hat{\theta}_{j}}^{\star}\left(\hat{\beta}_{j}\right)\right]_{+} \operatorname{sinal}\left(z_{j}\right), \operatorname{se}\left|z_{j}\right|>\Delta_{j}
\end{array}\right.
$$

No qual $z_{j}=\boldsymbol{X}_{j}^{\prime}\left(\boldsymbol{Y}-\sum_{k \neq j}\right) \boldsymbol{X}_{k} \hat{\beta}_{k}, \Delta_{j} \equiv \inf _{\beta_{j}>0}\left[n \beta_{j} / 2-\rho\left(\beta_{j} \mid \hat{\theta}_{j}\right) / \beta_{j}\right]$, o operador $[\ldots]_{+}$representa um problema de maximização e sinal (...) representa o sinal + ou - do operando e $\hat{\theta}_{j}=E\left(\theta \mid \hat{\boldsymbol{\beta}}_{\backslash j}\right)$.

Todas essas variáveis dependem do cálculo de $\hat{\theta}_{j}=E\left(\theta \mid \hat{\boldsymbol{\beta}}_{\backslash j}\right)$, por isso, será necessário também definir $P(\theta \mid \hat{\boldsymbol{\beta}})$. à partir de uma distribuição à priori Beta $(a, b)$, os autores chegam à seguinte distribuição condicional:

$$
P(\theta \mid \hat{\boldsymbol{\beta}}) \propto \theta^{a-1}(1-\theta)^{b-1}\left(1-\theta\left(1-\frac{\lambda_{1}}{\lambda_{0}}\right)\right)^{(p-\hat{q})} \prod_{j=1}^{\hat{q}}\left(1-\theta x_{j}\right),
$$

$\operatorname{com} x_{j}=\left(1-\frac{\lambda_{1}}{\lambda_{0}} \exp \left(\left|\hat{\beta}_{j}\right|\left(\lambda_{0}-\lambda_{1}\right)\right)\right)$. Os autores notam que $E\left(\theta \mid \hat{\boldsymbol{\beta}}_{\backslash j}\right)$ será muito próximo a $E(\theta \mid \hat{\boldsymbol{\beta}})$ para valores grandes de p, que por sua vez pode ser aproximada por ((ROČKOVÁ; GEORGE, 2016b, p. 16)):

$$
E(\theta \mid \hat{\boldsymbol{\beta}}) \approx \frac{B(a+1, b)}{B(a, b)} \frac{\left.F_{1}(a+1, \hat{q}-p,-\hat{q}, a+b+1 ; z, \boldsymbol{x})\right)}{F_{1}(a, \hat{q}-p,-\hat{q}, a+b ; z, \boldsymbol{x})} .
$$

Na qual $z=1-\frac{\lambda_{1}}{\lambda_{0}}$ e $F_{1}$ é a função $F_{1}$ de Appel, dada por:

$$
F_{1}\left(a^{\prime}, b^{\prime}, c^{\prime}, d^{\prime} ; z, x\right)=\frac{1}{B\left(d^{\prime}-a^{\prime}, a^{\prime}\right)} \int_{0}^{1} \theta^{a^{\prime}-1}(1-\theta)^{d^{\prime}-a^{\prime}-1}(1-\theta z)^{-b^{\prime}}(1-\theta x)-c^{\prime} d \theta
$$

Com esses resultados é possível definir o processo iterativo que levará à resolução do problema, dado por: 


$$
\beta_{j}^{(k+1)}=\tilde{S}\left(z_{j}^{(k)}, \lambda_{\tilde{\theta}_{j}^{(k)}}^{\star}\left(\beta_{j}^{k}\right), \Delta_{j}\right), \operatorname{com} \tilde{\theta}_{j}^{(k)}=E\left(\theta \mid \tilde{\boldsymbol{\beta}}_{\backslash j}^{(k)}\right),
$$

sendo $\tilde{S}\left(z^{\prime} \lambda, \Delta\right)=\frac{1}{n}(|z|-\lambda)_{+} \operatorname{sinal}(z) I(|z|>\Delta), I(\ldots)$ representando a função indicador. Além disso, os autores recomendam não atualizar $E(\theta \mid \hat{\boldsymbol{\beta}})$ a cada $\beta_{j}^{(k)}$, mas esperar $M$ valores de $j$ para fazê-lo. No exemplo discutido no artigo, $M=10$, ou seja, $E(\theta \mid \hat{\boldsymbol{\beta}})$ é recalculado em $j=11$, $j=21, j=31$, etc. Lembrando que essa metodologia foi desenvolvida para casos com muitas variáveis explicativas, inclusive casos com mais variáveis explicativas do que observações.

Além disso, os autores propõe criar uma grade de valores crescentes para $\lambda_{0}$, de modo que as estimativas possam ser comparadas e seja verificada a estacionariedade.

O algoritmo é dado da seguinte maneira (ROČKOVÁ; GEORGE, 2016b, p. 35, com adaptações):

(1) criar uma grade de valores crescentes de $\lambda_{0}, I=\left\{\lambda_{0}^{1}, \lambda_{0}^{2}, \ldots, \lambda_{0}^{L}\right\}$

(2) para cada $l \in\{1, \ldots, L\}$

(I) estabelecer $k=0$

(II) iniciar: $\boldsymbol{\beta}_{l}^{(k)}=\boldsymbol{\beta}^{\star}, \theta^{(0)}=\theta^{\star}$

(III) enquanto dif $>\epsilon$

(A) Incrementar $k$

(B) Para $s=1, \ldots, \frac{p}{M}$

(i) Para $j=1, \ldots, M$

(a) calcular $\Delta_{l(s-1) M+j}^{(k)} \operatorname{com} \theta=\theta^{(k-1)}$

(b) calcular $\beta_{l(s-1) M+j}^{(k)} \operatorname{com} \theta=\theta^{(k-1)}$

(ii) calcular $\theta^{(k)}=E\left(\theta \mid \boldsymbol{\beta}_{l}^{(k)}\right)$

(C) dif $=\mid \boldsymbol{\beta}^{(k)}-\boldsymbol{\beta}^{(k-1)} \|_{2}$

(IV) retornar $\boldsymbol{\beta}_{l}^{(k)}$

(V) fazer $\boldsymbol{\beta}^{\star}=\boldsymbol{\beta}_{l}^{(k)}$

Com isso é possível implementar o modelo do Spike-and-Slab LASSO com a penalidade não-separável. A vantagem desse método é que ele estima a moda da distribuição à posteriori do modelo com uma função de penalidade que se adapta ao nivel de esparsidade dos dados e que tem uma penalização diferente para cada uma das $j$ coordenadas do vetor $\boldsymbol{\beta}$ (os estudos de simulação desenvolvidos pelos autores indicam que a penalidade adaptativa se aproxima do caso de uma separável com a variável $\theta$ ajustada no nível real de esparsidade $\left.\frac{q}{p}\right)$. Além disso, a grade de valores $\lambda_{0}$ tem duas utilidades: Em primeiro lugar, é possível verificar se há uma grande diferença entre os valores $l$ ou se eles estão próximos, indicando uma estacionariedade, e em segundo, ela permite verificar o "caminho"da solução, o que segundo os autores, pode ser utilizado como uma ferramenta exploratória (ROČKOVÁ; GEORGE, 2016b, p. 19). 


\subsection{Aplicação de métodos bayesianos em genética}

Nos últimos anos os métodos bayesianos ganharam muita popularidade. Como exemplo, será brevemente discutido o trabalho de Li e Zhang (2010). Esse método combina vários elementos de métodos previamente apresentados nesse Capítulo, mas é focado em problemas específicos encontrados na pesquisa médica e genética.

Parte-se de um modelo linear similar aos já apresentados nesse Capítulo:

$$
\boldsymbol{Y}=\boldsymbol{X} \boldsymbol{\beta}+\boldsymbol{\epsilon}
$$

$\boldsymbol{Y}_{n \times 1}, \boldsymbol{X}=\left(\boldsymbol{X}_{1}, \boldsymbol{X}_{2}, \ldots, \boldsymbol{X}_{p}\right)$ é uma matriz $n \times p$, com $\boldsymbol{X}_{i}$ o vetor referente aos valores de cada variável explicativa. Assume-se que $p>n$, ou seja, existem mais variáveis explicativas do que observações, o que torna impossível o uso da regressão tradicional, além disso $\boldsymbol{\epsilon} \sim N\left(\mathbf{0}, \sigma^{2} I\right)$. Define-se o vetor de variáveis indicadoras $\gamma=\left(\gamma_{1}, \ldots, \gamma_{p}\right)$, que representa se a variável explicativa $X_{i}$ e o correspondente coeficiente $\beta_{i}$ está no modelo ou não. Da mesma maneira que Yuan e Lin (2005) e Ročková e George (2016b), e assim como em Park e Casella (2008a), é utilizado o amostrador de Gibbs para se calcular a mediana da distribuição à posteriori de $\gamma_{i}$. Nos dois outros trabalhos que utilizam a metodologia de uma distribuição à priori para $\gamma$, esta é considerada um produto de distribuições de Bernoulli (ou similares à Bernoulli com ponderações levemente diferentes). No trabalho de Li e Zhang (2010), a distribuição à priori de $\gamma$ é bastante diferente.

Os autores utilizam essa abordagem devido ao fato de que, em muitos problemas de genética e medicina, está se trabalhando com variáveis aleatórias explicativas com uma estrutura probabilística conhecida. Por exemplo: Em estudos de câncer, cromossomos adjacentes em geral estão relacionados aos mesmos defeitos genéticos; diagnósticos de imagem por meio de ressonância magnética cerebral envolvem partes do cérebro que estão conectadas entre si; e em sequenciamento genético existem estruturas chamadas "expressões"e "palavras"genéticas, que novamente, têm formas conhecidas. Em todos esses casos, o número de genes e cromossomos envolvidos é muito grande, mas o número de pacientes analisados é limitado. Assim, os autores propõe um modelo que utiliza uma distribuição à priori que incorpora a estrutura de correlação que pode existir entre variáveis explicativas associadas a medidas adjacentes (ou seja, entre $\gamma_{i}$ e seus dois "vizinhos", $\gamma_{i-1}$ e $\left.\gamma_{i+1}\right)$ :

$$
\begin{gathered}
\beta_{i} \mid \gamma_{i} \sim\left(1-\gamma_{i}\right) I_{0}+\gamma_{i} N\left(0, \sigma^{2} v^{2}\right), i=1,2, \ldots p, \\
\sigma^{2} \mid \gamma \sim G I\left(\frac{v}{2}, \frac{v \lambda}{2}\right), \\
\gamma \in\{0,1\}^{p} \\
P(\boldsymbol{\gamma})=\exp \left(\boldsymbol{a}^{\prime} \boldsymbol{\gamma}+\boldsymbol{\gamma}^{\prime} \boldsymbol{B} \boldsymbol{\gamma}-\psi(\boldsymbol{a}, \boldsymbol{B})\right),
\end{gathered}
$$

com GI representando uma distribuição gama inversa (definida anteriormente), $v$ e $\lambda$ seus parâmetros e $I_{0}=P\left(\beta_{i}=0\right)$,

$$
\psi(\boldsymbol{a}, \boldsymbol{B})=\ln \left(\sum_{\boldsymbol{\gamma} \in\{0,1\}^{p}} \exp \left(\boldsymbol{a}^{\prime} \boldsymbol{\gamma}+\boldsymbol{\gamma}^{\prime} \boldsymbol{B} \boldsymbol{\gamma}\right)\right),
$$


em que $\boldsymbol{a}=\left(a_{1}, \ldots, a_{p}\right)^{\prime}$ é um vetor de valores conhecidos maiores que zero, que controla a esparcidade e $\boldsymbol{B}=\left(b_{i, j}\right)_{p \times p}$ é uma matriz simétrica cujos valores representam a crença à priori da relação entre dois parâmetros vizinhos, e, quanto maior os valores dos parâmetros $\boldsymbol{a}$ e $B$, maior a relação. Parâmetros que se acredita serem não correlacionados recebem valor $b_{i, j}=0$. Os autores utilizam $\boldsymbol{a}=a(1,1, \ldots, 1)$ para representar que, à priori, nenhuma varável está mais ou menos propensa a entrar no modelo final e também que, nos casos em que a correlação específica de cada par de variáveis não é conhecida à priori, pode-se tomar:

$$
b_{i, j}=\left\{\begin{array}{l}
0, \text { se não se assume correlação entre as variáveis explicativas } X_{i} \text { e } X_{j} \\
b, \text { se existe correlação. }
\end{array}\right.
$$

Com isso, na falta de informações adicionais, a distribuição de $\gamma$ depende apenas dos parâmetros $a$ e $b$. Além disso, se $\boldsymbol{a}$ é um vetor com todos os elementos iguais a 1 e $\boldsymbol{B}=0$, a distribuição à priori de $\gamma$ se reduz a um produto de distribuições de Bernoulli. (Os autores notam que também é possível fazer essa representação por meio de grafos).

A distribuição $P(\gamma)$ é conhecida como priori de Ising e foi desenvolvida em estudos de Física Quântica. Uma de suas propriedades é que a distribuição condicional de $\gamma_{i}$, dados todos os outros $\gamma_{j}, j \neq i$ (a notação é $\left.\gamma_{i} \mid \gamma_{(-i)}\right)$ é dada por:

$$
P\left(\gamma_{i} \mid \gamma_{(-i)}\right)=\frac{\exp \left(\gamma_{i}\left(a+b \sum_{j \in I_{(-i)}} \gamma_{j}\right)\right)}{1+\exp \left(a+b \sum_{j \in I_{(-i)}} \gamma_{j}\right)}
$$

na qual $I_{(-i)}$ é o conjunto de índices composto por todos os índices no conjunto das $p$ variáveis explicativas exceto o índice $i$. Essa é a probabilidade da inclusão da i-ésima variável dado que as demais já estão no modelo. A distribuição à posteriori $\gamma \mid \boldsymbol{Y}$ pode ser obtida por meio do teorema Bayes, da forma:

$$
P\left(\gamma_{i}=1 \mid \gamma_{(-i)}, \boldsymbol{Y}\right)=\frac{P\left(\boldsymbol{Y} \mid \gamma_{i}=1, \gamma_{(-i)}\right) P\left(\gamma_{i}=1 \mid \gamma_{(-i)}\right)}{P\left(\boldsymbol{Y} \mid \gamma_{i}=1, \gamma_{(-i)}\right) P\left(\gamma_{i}=1 \mid \gamma_{(-i)}\right)+P\left(\boldsymbol{Y} \mid \gamma_{i}=0, \gamma_{(-i)}\right) P\left(\gamma_{i}=0 \mid \gamma_{(-i)}\right)}
$$

Dividindo-se o numerador e o denominador por $P\left(\boldsymbol{Y} \mid \gamma_{i}=1, \gamma_{(-i)}\right)$ obtém-se:

$$
P\left(\gamma_{i}=1 \mid \gamma_{(-i)}, \boldsymbol{Y}\right)=\frac{P\left(\gamma_{i}=1 \mid \gamma_{(-i)}\right)}{P\left(\gamma_{i}=1 \mid \gamma_{(-i)}\right)+F\left(i \mid \gamma_{(-i)}\right)^{-1} P\left(\gamma_{i}=0 \mid \gamma_{(-i)}\right)}
$$

$\operatorname{com} F\left(i \mid \gamma_{(-i)}\right)=\frac{P\left(\boldsymbol{Y} \mid \gamma_{i}=1, \gamma_{(-i)}\right)}{P\left(\boldsymbol{Y} \mid \gamma_{i}=0, \gamma_{(-i)}\right)}$ representando o fator de Bayes. Verifica-se que tal fator pode ser escrito diretamente como:

$$
F\left(i \mid \gamma_{(-i)}\right)=v^{-1} \frac{\left|A_{-i}\right|^{\frac{1}{2}}}{\left|A_{i}\right|^{\frac{1}{2}}}\left(\frac{Y^{\prime} Y-Y^{\prime} X_{I_{-i}} A_{-i}^{-1} X_{I_{-i}}^{\prime} Y}{Y^{\prime} Y-Y^{\prime} X_{I_{i}} A_{i}^{-1} X_{I_{i}}^{\prime} Y}\right)
$$

$\operatorname{com} A_{i}=X_{I i}^{\prime} X_{I i}+v^{-2} I_{p i}$ e $A_{(-i)}=X_{I(-i)}^{\prime} X_{I(-i)}+v^{-2} I_{p(-i)}$. $I_{i}$ é o conjunto de todos os índices $i$ em $p$ e $I_{(-i)}$ é o conjunto de todos os índices $j \neq i$. Já $I_{p i}$ e $I_{p(-i)}$ são matrizes identidade de ordem $p$ e $p-1$, respectivamente. 
Assim, pode-se gerar uma cadeia de Markov (cuja matriz de transição será especificada posteriormente) no espaço $\{0,1\}^{p}$ para amostrar a distribuição à posteriori: Cria-se a cadeia de $\gamma_{i}^{\prime} s$ e para cada $\gamma_{i}$ calcula-se $P\left(\gamma_{i}=1 \mid \gamma_{(-i)}, \boldsymbol{Y}\right)$ utilizando (4.25). A partir da amostragem dessa distribuição pode-se realizar a seleção de modelos da seguinte maneira:

Amostra-se, para cada $\gamma_{i}$, a distribuição $P\left(\gamma_{i} \mid \gamma_{(-i)}, \boldsymbol{Y}\right)$ e calcula-se sua mediana. Caso essa mediana seja maior que 0,5 , a variável $i$ é incluída no modelo final. Os autores comentam sobre a complexidade do cálculo da expressão (4.26) e apresentam algumas soluções aproximadas para o problema. Destacam ainda que, embora a abordagem bayesiana não limite o "tamanho do modelo", um valor alto dessa quantidade pode ser problemático. Por outro lado, o "tamanho do modelo" é fortemente influenciado pela escolha dos hiper-parâmetros da distribuição de Ising e da distribuição da variância $\sigma^{2}$. Em particular os autores notam que, em um estudo de simulação, valores para $b$ menores que 1,35 resultavam em modelos com entre 150 e 200 variáveis explicativas, porém quando o valor de $b$ foi fixado em 1,35 o número de variáveis explicativas escolhidas saltou para mais de 8000, estabilizando-se em torno desse valor para valores de $b$ maiores. Por outro lado, o aumento dos valores de $v$ e do número de observações $n$ levaram a uma diminuição no tamanho do modelo final.

Assim, para aplicar esse método é necessário estabelecer valores para três hiper-parâmetros: $v, a$ e $b$ (os autores não discutem os valores do parâmetro $\lambda$ ). O parâmetro $v$ é escolhido da seguinte maneira: Para cada $X_{i}$ efetua-se uma regressão linear simples de $Y$ em função de $X_{i}$ e estima-se a variância de $\hat{\beta}_{i}$ e, a partir dessas estimativas de variância determina-se um valor para $v$. Uma abordagem alternativa e mais complexa seria utilizar uma abordagem hierárquica e estabelecer uma distribuição para $v$. Os autores sugerem uma distribuição bimodal com um pico em 0 e uma cauda contínua à direita. Esse tipo de mistura pode ser considerado uma distribuição do tipo Spike-and-Slab (LI; ZHANG, 2010, p. 1205). A escolha dos valores de $a$ e $b$ tem grande influência no tamanho do modelo final e é estudada pelos autores em detalhes, mas, para a aplicação prática, deve-se utilizar a informação à priori contida na distribuição de Ising para criar a cadeia de Markov que é utilizada no amostrador de Gibbs.

A matriz de transição de uma cadeia de Markov com dois estados tem a forma genérica:

$$
Q=\left[\begin{array}{ll}
q_{0} & 1-q_{0} \\
q_{1} & 1-q_{1}
\end{array}\right],
$$

ou seja, $P\left(\gamma_{i+1}=0 \mid \gamma_{i}=0\right)=q_{0}$ e $P\left(\gamma_{i+1}=0 \mid \gamma_{i}=1\right)=q_{1}$, e tal que $\gamma_{1}$ tem distribuição de Bernoulli com parâmetro $\frac{1-q_{0}}{2-q_{0}-q_{1}}$, sendo que $\left(\frac{1-q_{1}}{2-q_{0}-q_{1}}, \frac{1-q_{0}}{2-q_{0}-q_{1}}\right)$ é a distribuição estacionária associada à cadeia de Markov com matriz de transição Q. Nessas condições:

$$
\left[\frac{1-q_{1}}{2-q_{0}-q_{1}}, \frac{1-q_{0}}{2-q_{0}-q_{1}}\right] \times Q=\left[\frac{1-q_{1}}{2-q_{0}-q_{1}}, \frac{1-q_{0}}{2-q_{0}-q_{1}}\right],
$$

ou seja:

$$
\lim _{n \rightarrow \infty}\left[P\left(\gamma_{n}=0\right), P\left(\gamma_{n}=1\right)\right]=\left[\frac{1-q_{1}}{2-q_{0}-q_{1}}, \frac{1-q_{0}}{2-q_{0}-q_{1}}\right]
$$


Define-se:

$$
\begin{aligned}
r & =\frac{1-q_{0}}{\left(1-q_{1}\right)} \\
w_{0} & =\frac{q_{0}}{\left(1-q_{1}\right)} \\
w_{1} & =\frac{q_{1}}{\left(1-q_{0}\right)}
\end{aligned}
$$

Esses termos tem interpretações que auxiliam na tarefa de especificar a distribuição à priori de $\gamma: r$ representa a chance (isto é, a razão de probabilidades) à priori de $\gamma_{i}=1$ com relação a $\gamma_{i}=0, w_{0}$ reflete o acréscimo na probabilidade de $\gamma_{i}=0$ dado que sabe-se que $\gamma_{i-1}=0$ e $w_{1}$ reflete o acréscimo na probabilidade de $\gamma_{i}=1$ dado $\gamma_{i-1}=1$.

Agora é necessário voltar à distribuição de Ising. A distribuição condicional de $\gamma_{i}$, dado $\gamma_{i-1}$ e $\gamma_{i+1}$ é:

$$
P\left(\gamma_{i}=1 \mid \gamma_{i-1}, \gamma_{i+1}\right)=\frac{\exp \left(a+b\left(\gamma_{i-1}+\gamma_{i+1}\right)\right)}{1+\exp \left(a+b\left(\gamma_{i-1}+\gamma_{i+1}\right)\right)}
$$

Pode-se então estabelecer os parâmetros $a$ e $b$ tomando:

$$
\begin{aligned}
& a=\ln \left(\frac{r}{w_{0}^{2}}\right), \mathrm{e} \\
& b=\ln \left(w_{1} w_{0}\right) .
\end{aligned}
$$

Com isso, a partir dos valores $a$ e $b$ torna-se possível construir uma matriz de transição da forma especificada em (4.27) e construir a cadeia de Markov necessária para aplicar o modelo. Os autores notam que, ao invés de utilizar $a$ e $b$, também é possível trabalhar apenas especificando $(r, w)$.

A partir da cadeia de Markov, é possível calcular a mediana de cada parâmetro utilizando o amostrador de Gibbs e seleciona-se como modelo final aquele em que entram todas as variáveis cuja mediana da distribuição à posteriori $P\left(\gamma_{i}=1 \mid \boldsymbol{Y}\right)$ é maior ou igual a 0,5 . 


\section{Aplicações e simulação}

\subsection{Introdução}

Neste Capítulo, busca-se fazer uma comparação entre os seguintes métodos discutidos anteriormente: NAMS, RFS, LARS, LASSO Bayesiano ((PARK; CASELLA, 2008a)) e Spikeand-Slab LASSO.

Os métodos do LASSO bayesiano e do LARS já estão implementados em pacotes do $\mathrm{R}^{1}$, o Spike-and-Slab LASSO tem uma implementação em R disponibilizado no site da autora ${ }^{2}$. Os autores do NAMS e do método da RFS também disponibilizaram um código em $\mathrm{R}^{3}$. O código para a RFS inclui algumas modificações teóricas posteriores, por isso escolheu-se não utilizar o código e ao invés disso desenvolver um código próprio e independente. O código do NAMS foi escrito em uma versão antiga do $\mathrm{R}$ e foram necessários alguns ajustes. $\mathrm{O}$ código da RFS e os ajustes no código do NAMS são detalhados no apêndice B. A RFS foi definida para $5 \%$ de razão de falsa seleção.

A comparação é feita em duas situações: com bases de dados reais e com dados advindos de uma simulação simulação.

\subsection{Bases de dados reais}

Nos exemplos a seguir os métodos são aplicados em duas bases reais e calcula-se o tamanho (isto é, número de variáveis explicativas inclusas no modelo) e o quadrado médio dos resíduos (QMR), definido como $\frac{\sum_{i=1}^{n}\left(y_{i}-\hat{y}_{i}\right)^{2}}{n-k}$, no qual $n$ é o número de observações e $k$ o número de variáveis explicativas inclusas no modelo. QMR será utilizado para comparar a qualidade do ajuste.

\subsubsection{Atitude}

A base atitude foi apresentada por Chatterjee, Hadi e Price (1999) e é composta de 30 observações de sete variáveis, sendo a variável resposta uma nota atribuída a gestores de uma empresa e as variáveis explicativas são relacionadas às atitudes do gestor para com seus funcionários.

A Tabela 1 apresenta os resultados da estimação:

\footnotetext{
pacotes monomvn e lars, respectivamente disponível em:

$<$ http://faculty.chicagobooth.edu/veronika.rockova/>

3 diponível em:

<http://www4.stat.ncsu.edu/ boos/var.select/>
} 


\begin{tabular}{ccc}
\hline Método & \# de var. sel. & QMR \\
\hline NAMS & 1 & 4368,738 \\
RFS & 1 & 4368,738 \\
LARS & 2 & 4521,763 \\
LASSO bayesiano & 2 & 4528,627 \\
Spike-and-Slab LASSO & 5 & 4517,026 \\
\hline
\end{tabular}

Tabela 1 - Medidas associadas à base atitude

O NAMS e a RFS escolheram o mesmo modelo. Além disso, o LARS e o LASSO também selecionaram o mesmo modelo, que inclusive é o modelo selecionado por Chatterjee, Hadi e Price (1999).

\subsubsection{Diabetes}

A base de dados da Diabetes possui uma variável resposta, denominada Y, 10 variáveis explicativas relacionadas a idade, sexo, índice de massa corpórea e medidas colhidas em exames de sangue. A base contém 442 observações e foi introduzida por (EFRON et al., 2004), no contexto do LARS.

Os resultados encontram-se na Tabela 2 :

\begin{tabular}{ccc}
\hline Método & \# de var. sel. & QMR \\
\hline NAMS & 6 & 26379,37 \\
RFS & 6 & 26379,37 \\
LARS & 7 & 26448,89 \\
LASSO bayesiano & 6 & 26408,71 \\
Spike-and-Slab LASSO & 10 & 26606,26 \\
\hline
\end{tabular}

Tabela 2 - Medidas associadas à base diabetes

Novamente, o NAMS e o RFS escolheram o mesmo modelo, que também foi o mesmo do LASSO Bayesiano.

\subsubsection{Comparação}

Nos dois casos, percebe-se que os métodos NAMS e RFS selecionaram os modelos com menos variáveis explicativas. O LARS gerou modelos com mais variáveis explicativas, porém com um ajuste pior. O Spike-and-Slab LASSO produziu os maiores modelos, mas, em termos de qualidade do ajuste (pelo quadrado médio do resíduo), se saiu melhor que o LASSO Bayesiano no primeiro caso e um pouco pior no segundo. Do ponto de vista computacional, o método mais veloz é o LARS e os mais lentos são os métodos baseados em pseudovariáveis, com o NAMS sendo o mais lento de todos. 


\subsection{Simulação}

O estudo de simulação foi feito da seguinte maneira: Foram criadas três matrizes de variáveis explicativas, cada uma com 20 variáveis explicativas $\boldsymbol{X}_{1}, \boldsymbol{X}_{2}, \ldots, \boldsymbol{X}_{20}$ e 100 observações de cada variável. Isso foi feito por meio de uma simulação de uma distribuição Normal Multivariada com $\boldsymbol{\mu}_{20}$ um vetor nulo (ou seja, cada uma das variáveis tem média zero) e uma estrutura de covariância diferente para cada uma delas: Na primeira, a covariância entre as variáveis $i$ e $j$ é $\operatorname{cov}\left(\boldsymbol{X}_{i}, \boldsymbol{X}_{j}\right)=0, i \neq j$, na segunda $\operatorname{cov}\left(\boldsymbol{X}_{i}, \boldsymbol{X}_{j}\right)=0,3^{|i-j|}$ e na terceira $\operatorname{cov}\left(\boldsymbol{X}_{i}, \boldsymbol{X}_{j}\right)=0,7^{|i-j|}$.

Depois disso, foram criados três grupos de coeficientes $\boldsymbol{\beta}$, com 20 componentes, associados às 20 variáveis preditoras. No primeiro grupo, 5 dos 20 coeficientes são não-nulos. Esses valores foram gerados à partir de uma distribuição Uniforme que sorteou 5 valores inteiros entre $1 \mathrm{e}$ 10. A posição desses números no vetor foi aleatorizada. O mesmo foi feito para os dois grupos seguintes, mas com 10 e 15 valores não nulos. Dentro de cada um dos três grupos de covariância, os índices e valores dos coeficientes não-nulos são os mesmos.

A seguir, foram criados nove grupos de variáveis resposta, cada grupo com 100 vetores. Elas foram criadas da seguinte forma $\boldsymbol{Y}_{i, j, k}=\boldsymbol{X}_{i, j} \boldsymbol{\beta}_{j}+\boldsymbol{\epsilon}_{i, j, k}$, com $\boldsymbol{\epsilon}_{i, j, k} \sim N(0,1)$, no qual cada $i$ está associado a cada uma das três estruturas de correlação, $j$ está relacionada a um dos três conjuntos de coeficientes e $k=1, \ldots, 100$, totalizando 900 vetores de 100 observações cada.

Essa estrutura de simulação é similar à estrutura proposta por Luo, Stefanski e Boos (2006), o artigo que propõe o procedimento NAMS.

Nesse estudo, são comparados o tamanho médio do modelo selecionado por cada método com base nos 100 conjuntos de dados, quantas vezes cada um dos preditores com coeficientes não nulos é selecionado para o modelo final e o percentual de vezes em que o método selecionou todas as variáveis explicativas corretas e apenas elas.

Além disso, também foi estimado o erro quadrático médio (EQM) do vetor $\boldsymbol{\beta}$ para cada método de seleção e caso de correlação. O erro quadrático médio é definido como $\mathrm{E}_{\boldsymbol{\theta}}\left[(\hat{\boldsymbol{\theta}}-\boldsymbol{\theta})^{2}\right]$. No presente caso, EQM foi estimado como $\frac{\sum_{j}^{100} \sum_{i=1}^{p}\left(\hat{\theta}_{i, j}-\theta_{i}\right)^{2}}{100}$, em que $\hat{\theta}_{i, j}$ é a estimativa de $\beta_{j}$ na $j$-ésima repetição. Ao final de cada um dos três grupos (5, 10 e 15 variáveis explicativas), apresentam-se tabelas resumo indicando quais eram as variáveis não nulas, quantas vezes cada variável foi selecionada em cada método em cada uma das três estruturas de covariância.

\subsubsection{Cinco coeficientes das variáveis explicativas não nulos}

Nas bases de simulação com cinco variáveis explicativas não nulas, o índice das variáveis explicativas diferentes de zero foi: $1,3,12,15$ e 16, e seus coeficientes são, respectivamente, 2, 2, $4,3,3$. O resultado de cada método será analisado a seguir.

\subsubsection{NAMS}

Sem nenhuma estrutura de correlação, dentre as 100 repetições do procedimento, o NAMS selecionou em média 5,05 variáveis explicativas para o modelo final. Com a estrutura de correlação baixa, foram selecionadas em média 5,20 variáveis explicativas para o modelo final 
e com a estrutura de correlação alta foram selecionadas, em média, 5,39 variáveis explicativas. Assim, neste estudo de simulação, a estrutura de correlação entre as variáveis explicativas não teve grande efeito na seleção do modelo.

Como se pode verificar, em todos os 100 casos, todas as variáveis explicativas com coeficientes não-nulos foram selecionadas corretamente, com algumas variáveis explicativas com coeficientes nulos sendo selecionados esporadicamente.

\subsubsection{RFS}

Sem nenhuma estrutura de correlação dentre as 100 repetições do procedimento, a RFS selecionou em média, 5,14 variáveis explicativas não nulas para o modelo final. Com a estrutura de correlação baixa selecionou, em média, 5,16 variáveis e com a estrutura de correlação alta, 5,09 variáveis. Assim, esse método também se mostrou robusto ao aumento da correlação entre variáveis explicativas.

Novamente, todas as variáveis explicativas relevantes foram selecionadas em todos os modelos.

\subsubsection{LARS}

No caso em que as variáveis explicativas são não correlacionadas o LARS selecionou em média 10,48 variáveis pelo critério do menor $C_{p}$. Com a estrutura de correlação baixa, selecionou em média 10,74 variáveis e com a estrutura de correlação alta selecionou em média 10,38 variáveis. Apesar de não ter sido constatada uma diferença grande entre as estruturas de correlação, nota-se que o LARS tem uma tendencia a superestimar o número de variáveis explicativas não-nulas.

Esse resultado inspirou uma nova análise: analisar os resultados do LARS, caso o procedimento fosse interrompido na etapa igual ao número de variáveis explicativas com coeficientes não-nulos. Esse estudo tem interesse puramente comparativo, porque em uma aplicação real não se sabe à priori o número real de variáveis explicativas relevantes.

Os resultados desse cálculo serão chamados de "LARS - tamanho de modelo fixo", e seus resultados serão apresentados no final.

\subsubsection{LASSO Bayesiano}

No caso sem correlação entre as variáveis explicativas, o LASSO bayesiano selecionou em média 5,37 variáveis explicativas, no caso com a correlação baixa entre as variáveis, selecionou em média 5,41 variáveis e com a correlação alta foram selecionadas em média 5,42.

\subsubsection{Spike-and-Slab LASSO - SSL}

No caso em que as variáveis explicativas são não correlacionadas o SSL selecionou as 5,01 variáveis. Com uma correlação baixa, o SSL selecionou somente as 5 variáveis corretas todas as vezes e com a correlação alta o tamanho médio foi 5,01. O SSL teve a melhor precisão na seleção de variáveis entre todos os métodos no caso com 5 variáveis explicativas (a precisão na estimação será comparada a seguir). 


\subsubsection{Comparação}

A tabela 3 destaca o número de vezes que cada uma das variáveis foi selecionada para cada método e em cada estrutura de correlação. "LARSF"se refere ao "LARS - Tamanho Fixo", "BLASSO"é o LASSO Bayesiano e "SSL"o Spike-and-Slab LASSO.

\begin{tabular}{|c|c|c|c|c|c|c|c|c|c|c|c|c|c|c|c|c|c|c|c|c|c|}
\hline Variável & & 1 & 2 & 3 & 4 & 5 & 6 & 7 & 8 & 9 & 10 & 11 & 12 & 13 & 14 & 15 & 16 & 17 & 18 & 19 & 20 \\
\hline \multirow[t]{6}{*}{ Sem correlação } & NAMS & 100 & 0 & 100 & 1 & 0 & 1 & 0 & 1 & 0 & 0 & 0 & 100 & 0 & 0 & 100 & 100 & 1 & 0 & 0 & 1 \\
\hline & RFS & 100 & 1 & 100 & 1 & 4 & 0 & 0 & 2 & 2 & 1 & 1 & 100 & 0 & 0 & 100 & 100 & 1 & 0 & 0 & 1 \\
\hline & LARS & 100 & 38 & 100 & 39 & 36 & 36 & 38 & 39 & 38 & 32 & 37 & 100 & 37 & 45 & 100 & 100 & 34 & 25 & 41 & 33 \\
\hline & LARSF & 100 & 0 & 100 & 0 & 0 & 0 & 0 & 0 & 0 & 0 & 0 & 100 & 0 & 0 & 100 & 100 & 0 & 0 & 0 & 0 \\
\hline & BLASSO & 100 & 1 & 100 & 1 & 6 & 2 & 1 & 6 & 3 & 2 & 3 & 100 & 2 & 1 & 100 & 100 & 3 & 1 & 1 & 4 \\
\hline & SSL & 100 & 0 & 100 & 0 & 0 & 0 & 0 & 0 & 0 & 0 & 0 & 100 & 0 & 0 & 100 & 100 & 1 & 0 & 0 & 0 \\
\hline \multirow[t]{6}{*}{ Cor. baixa } & NAMS & 100 & 1 & 100 & 1 & 1 & 1 & 5 & 0 & 2 & 1 & 1 & 100 & 1 & 1 & 100 & 100 & 1 & 1 & 0 & 3 \\
\hline & RFS & 100 & 0 & 100 & 1 & 2 & 0 & 3 & 1 & 0 & 1 & 1 & 100 & 2 & 0 & 100 & 100 & 0 & 1 & 0 & 4 \\
\hline & LARS & 100 & 36 & 100 & 55 & 25 & 42 & 30 & 37 & 38 & 35 & 37 & 100 & 37 & 37 & 100 & 100 & 36 & 36 & 41 & 52 \\
\hline & LARSF & 100 & 0 & 100 & 0 & 0 & 0 & 0 & 0 & 0 & 0 & 0 & 100 & 0 & 0 & 100 & 100 & 0 & 0 & 0 & 0 \\
\hline & BLASSO & 100 & 1 & 100 & 2 & 3 & 2 & 5 & 5 & 1 & 1 & 3 & 100 & 2 & 1 & 100 & 100 & 4 & 3 & 1 & 7 \\
\hline & SSL & 100 & 0 & 100 & 0 & 0 & 0 & 0 & 0 & 0 & 0 & 0 & 100 & 0 & 0 & 100 & 100 & 0 & 0 & 0 & 0 \\
\hline \multirow[t]{6}{*}{ Cor. Alta } & NAMS & 100 & 4 & 100 & 2 & 1 & 3 & 4 & 2 & 3 & 2 & 2 & 100 & 4 & 1 & 100 & 100 & 3 & 3 & 2 & 3 \\
\hline & RFS & 100 & 3 & 100 & 0 & 0 & 1 & 0 & 0 & 2 & 1 & 0 & 100 & 0 & 0 & 100 & 100 & 0 & 0 & 0 & 2 \\
\hline & LARS & 100 & 57 & 100 & 32 & 21 & 30 & 34 & 24 & 33 & 35 & 41 & 100 & 35 & 44 & 100 & 100 & 41 & 41 & 35 & 35 \\
\hline & LARSF & 100 & 3 & 97 & 0 & 0 & 0 & 0 & 0 & 0 & 0 & 0 & 100 & 0 & 0 & 100 & 100 & 0 & 0 & 0 & 0 \\
\hline & BLASSO & 100 & 5 & 100 & 2 & 0 & 2 & 7 & 2 & 3 & 2 & 5 & 100 & 3 & 4 & 100 & 100 & 1 & 2 & 0 & 4 \\
\hline & SSL & 100 & 1 & 100 & 0 & 0 & 0 & 0 & 0 & 0 & 0 & 0 & 100 & 0 & 0 & 100 & 100 & 0 & 0 & 0 & 0 \\
\hline
\end{tabular}

Tabela 3 - 5 variáveis explicativas

Nota-se que, com exceção do "LARS - tamanho fixo", todos os métodos selecionaram todas as variáveis corretas todas as vezes. Esse resultado será discutido mais adiante, mas, nessas condições, os critérios que serão utilizados para comparar os métodos e definir qual o "melhor"serão o menor tamanho médio do modelo, o menor $\widehat{E Q M}$ e o percentual de vezes em que são selecionadas as variáveis corretas e somente elas. Esses resultados são apresentados na tabela 4: 


\begin{tabular}{cccccc}
\hline Caso & Método & \# médio de var. sel. & d. p. \# & $\widehat{E Q M}$ & \% correto \\
\hline Não correlacionadas & NAMS & 5,05 & 0,2972942 & 0,05925797 & 97 \\
& RFS & 5,14 & 0,3765875 & 0,06759437 & 87 \\
& LARS & 10,48 & 3,2144874 & 0,16313122 & 1 \\
& LARS - TF & 5,00 & 0 & 0,45322525 & 100 \\
& LASSO B. & 5,37 & 0,6138963 & 0,06896832 & 70 \\
& SSL & 5,01 & 0,1000000 & 0,05699657 & 99 \\
\hline Correlação Baixa & NAMS & 5,20 & 0,9639984 & 0,07738393 & 91 \\
& RFS & 5,16 & 0,3949172 & 0,07888125 & 85 \\
& LARS & 10,74 & 1,2589478 & 0,17093721 & 0 \\
& LARS - TF & 5 & 0 & 0,61301709 & 100 \\
& LASSO B. & 5,41 & 0,6528106 & 0,08461885 & 67 \\
& SSL & 5,00 & 0 & 0,06348241 & 100 \\
\hline \multirow{2}{*}{ Correlação Alta } & NAMS & 5,39 & 1.8140585 & 0,12148549 & 93 \\
& RFS & 5,09 & 0,3208260 & 0,10361135 & 92 \\
& LARS & 10,38 & 2,3859014 & 0,26124004 & 0 \\
& LARS - TF & 5 & 0 & 1,41947719 & 97 \\
& LASSO B. & 5,42 & 0,6693884 & 0,13608431 & 67 \\
& SSL & 5,01 & 0,1 & 0,09391447 & 99 \\
\hline
\end{tabular}

Tabela 4 - Comparação dos métodos - 5 variáveis explicativas

Em todos os casos, o Spike-and-Slab LASSO apresentou o menor tamanho de modelo, o que, como todos os estimadores escolheram todas as variáveis corretas todas as vezes, também indica melhores seleções, e o menor $\widehat{E Q M}$ (excetuando um caso em que a construção teórica do LARS com número de coeficientes fixos foi melhor), indicando também uma maior precisão na estimação dos coeficientes.

Quanto ao percentual de acerto, o cálculo teórico do LARS com um número fixo de coeficientes foi o melhor quando não havia correlação entre os preditores, empatou com o SSL quando a estrutura de correlação era baixa e quando a estrutura de correlação era alta o SSL se saiu melhor. O SSL também foi o melhor no caso sem estrutura de correlação entre as metodologias que podem ser implementadas na prática.

Nota-se porém, que os resultados de três dos métodos: RFS, NAMS e SSL foram bastante próximos.

\subsubsection{0 variáveis explicativas}

Com 10 variáveis explicativas, os índices das variáveis cujos coeficientes são não-nulos foram: $2,4,5,7,8,10,11,12,15$, 20. Seus coeficientes são, respectivamente: $4,3,2,3,8,8,8,8$, $3,8$.

\subsubsection{NAMS}

Sem nenhuma estrutura de correlação, o NAMS selecionou em média 10,05 variáveis explicativas, com estrutura de correlação baixa selecionou 10,13 variáveis e com a estrutura de 
correlação alta selecionou 10,17 variáveis. Novamente, o impacto da estrutura de correlações foi baixo.

\subsubsection{RFS}

Sem estrutura de correlação, o RFS selecionou em média 10,08 variáveis, com a estrutura de correlação baixa, selecionou em média 10,16 variáveis e com a estrutura alta de correlação foram selecionadas 10,18 variáveis.

\subsubsection{LARS}

Sem estrutura de correlação, o LARS selecionou em média 15,15 variáveis, com a estrutura baixa de correlação, selecionou em média 14,79 variáveis e com a estrutura alta selecionou em média 14,99 variáveis.

Novamente, foi feito o estudo quando o número de coeficientes selecionados pelo LARS for fixo no número correto de coeficientes não nulos.

\subsubsection{LASSO Bayesiano}

No caso sem correlação, o Lasso Bayesiano selecionou em média 10,06 variáveis, com a estrutura de correlação baixa, selecionou em média 10,15 variáveis e com a estrutura de correlação alta selecionou em média 10,12 variáveis.

\subsubsection{Spike-and-Slab LASSO}

No caso sem correlação entre as variáveis explicativas, o SSL selecionou as 10,00 variáveis certas todas as vezes, com a correlação baixa entre as variáveis, 10,04, e com a correlação alta, também 10,04.

\subsubsection{Comparação}

Para o caso de 10 variáveis explicativas foram elaboradas as mesmas Tabelas do caso de 5 variáveis:

\begin{tabular}{|c|c|c|c|c|c|c|c|c|c|c|c|c|c|c|c|c|c|c|c|c|c|}
\hline Variável & & 1 & 2 & 3 & 4 & 5 & 6 & 7 & 8 & 9 & 10 & 11 & 12 & 13 & 14 & 15 & 16 & 17 & 18 & 19 & 20 \\
\hline \multirow[t]{6}{*}{ Sem correlação } & NAMS & 0 & 100 & 0 & 100 & 100 & 0 & 100 & 100 & 3 & 100 & 100 & 100 & 1 & 0 & 100 & 0 & 0 & 0 & 1 & 100 \\
\hline & RFS & 1 & 100 & 1 & 100 & 100 & 0 & 100 & 100 & 3 & 100 & 100 & 100 & 0 & 1 & 100 & 2 & 0 & 0 & 0 & 100 \\
\hline & LARS & 53 & 100 & 52 & 100 & 100 & 48 & 100 & 100 & 48 & 100 & 100 & 100 & 43 & 56 & 100 & 54 & 61 & 46 & 54 & 100 \\
\hline & LARSF & 0 & 100 & 0 & 100 & 100 & 0 & 100 & 100 & 0 & 100 & 100 & 100 & 0 & 0 & 100 & 0 & 0 & 0 & 0 & 100 \\
\hline & BLASSO & 0 & 100 & 1 & 100 & 100 & 0 & 100 & 100 & 4 & 100 & 100 & 100 & 0 & 0 & 100 & 1 & 0 & 0 & 0 & 100 \\
\hline & SSL & 0 & 100 & 0 & 100 & 100 & 0 & 100 & 100 & 0 & 100 & 100 & 100 & 0 & 0 & 100 & 0 & 0 & 0 & 0 & 100 \\
\hline \multirow[t]{6}{*}{ Cor. baixa } & NAMS & 1 & 100 & 1 & 100 & 100 & 1 & 100 & 100 & 2 & 100 & 100 & 100 & 3 & 2 & 100 & 3 & 0 & 0 & 0 & 100 \\
\hline & RFS & 2 & 100 & 1 & 100 & 100 & 2 & 100 & 100 & 2 & 100 & 100 & 100 & 3 & 2 & 100 & 3 & 0 & 0 & 1 & 100 \\
\hline & LARS & 42 & 100 & 45 & 100 & 100 & 52 & 100 & 100 & 56 & 100 & 100 & 100 & 43 & 43 & 100 & 47 & 52 & 48 & 51 & 100 \\
\hline & LARSF & 0 & 100 & 0 & 100 & 100 & 0 & 100 & 100 & 0 & 100 & 100 & 100 & 0 & 0 & 100 & 0 & 0 & 0 & 0 & 100 \\
\hline & BLASSO & 2 & 100 & 1 & 100 & 100 & 2 & 100 & 100 & 2 & 100 & 100 & 100 & 3 & 2 & 100 & 3 & 0 & 0 & 0 & 100 \\
\hline & SSL & 0 & 100 & 1 & 100 & 100 & 1 & 100 & 100 & 1 & 100 & 100 & 100 & 0 & 0 & 100 & 1 & 0 & 0 & 0 & 100 \\
\hline \multirow[t]{6}{*}{ Cor. Alta } & NAMS & 0 & 100 & 2 & 100 & 100 & 9 & 100 & 100 & 1 & 100 & 100 & 100 & 0 & 2 & 100 & 1 & 1 & 0 & 1 & 100 \\
\hline & RFS & 0 & 100 & 2 & 100 & 100 & 9 & 100 & 100 & 2 & 100 & 100 & 100 & 0 & 1 & 100 & 1 & 1 & 0 & 2 & 100 \\
\hline & LARS & 40 & 100 & 54 & 100 & 100 & 100 & 100 & 100 & 51 & 100 & 100 & 100 & 45 & 50 & 100 & 41 & 37 & 34 & 47 & 100 \\
\hline & LARSF & 0 & 100 & 0 & 100 & 100 & 100 & 78 & 100 & 0 & 100 & 100 & 100 & 0 & 0 & 22 & 0 & 0 & 0 & 0 & 100 \\
\hline & BLASSO & 0 & 100 & 2 & 100 & 100 & 3 & 100 & 100 & 2 & 100 & 100 & 100 & 1 & 1 & 100 & 1 & 0 & 0 & 2 & 100 \\
\hline & SSL & 0 & 100 & 0 & 100 & 100 & 1 & 100 & 100 & 1 & 100 & 100 & 100 & 0 & 1 & 100 & 1 & 0 & 0 & 0 & 100 \\
\hline
\end{tabular}

Tabela 5 - 10 variáveis explicativas 
Novamente, com exceção do "LARS - Tamanho Fixo", todos os métodos selecionaram todas as variáveis corretas todas as vezes. A comparação foi a seguinte:

\begin{tabular}{cccccc}
\hline Caso & Método & \# médio de var. sel. & d. p. \# & $\widehat{E Q M}$ & \% correto \\
\hline não correlacionadas & NAMS & 10,05 & 0,2611165 & 0,12308636 & 96 \\
& RFS & 10,08 & 0,2726599 & 0,12648701 & 92 \\
& LARS & 15,15 & 2,1666667 & 0,22498334 & 0 \\
& LARS - TF & 10,00 & 0 & 1,00749720 & 100 \\
& LASSO B. & 10,06 & 0,2386833 & 0,12002143 & 94 \\
& SSL & 10,00 & 0 & 0,11927046 & 100 \\
\hline Correlação Baixa & NAMS & 10,13 & 0,3666667 & 0,15103249 & 95 \\
& RFS & 10,16 & 0,3949172 & 0,15321565 & 85 \\
& LARS & 14,79 & 2,3150583 & 0,25434446 & 1 \\
& LARS - TF & 10 & 0 & 2,12310818 & 100 \\
& LASSO B. & 10,15 & 0,3859921 & 0,14608298 & 86 \\
& SSL & 10,04 & 0,2428784 & 0,14188637 & 97 \\
\hline \multirow{2}{*}{ Correlação Alta } & NAMS & 10,17 & 0,4033947 & 0,27003661 & 84 \\
& RFS & 10,18 & 0,4114522 & 0,27260745 & 83 \\
& LARS & 14,99 & 1,8613289 & 0,45596085 & 0 \\
& LARS - TF & 10 & 0 & 27,67859841 & 0 \\
& LASSO B. & 10,12 & 0,3265986 & 0,26550371 & 88 \\
& SSL & 10,04 & 0,1969464 & 0,25692488 & 96 \\
\hline
\end{tabular}

Tabela 6 - Comparação dos métodos - 10 variáveis

Também neste caso o SSL se saiu melhor nos quesitos tamanho médio do modelo e $\widehat{E Q M}$ em todos os casos.

No percentual de acerto do modelo selecionado, o SSL novamente foi o melhor em todos os casos (exceto no caso de variáveis explicativas pouco correlacionadas, no qual ficou um pouco atrás da construção teórica do LARS com o número de coeficientes fixos), seguido pelo LASSO Bayesiano, nos casos altamente correlacionados, e do NAMS no caso de variáveis explicativas não correlacionadas ou com correlação baixa.

O LARS com o número de coeficientes fixos mostrou um resultado surpreendente: Nenhum dos modelos escolhidos foi o correto no caso de variáveis explicativas com correlação alta. Isso se deve ao fato de que todos os modelos selecionaram a variável 6, que é incorreta. Isso provavelmente se deve ao fato de a variável 6 ser altamente correlacionada às variáveis 5 e 7 , que têm um coeficientes muito altos em relação aos demais.

\subsubsection{5 variáveis explicativas}

Com 15 variáveis explicativas, as variáveis com coeficientes não nulos são: 1, 3, 4, 5, 6, 7, $8,9,14,15,16,17,18,19$ e 20. Seus coeficientes, são respectivamente: 1, 7, 7, 6, 4, 7, 10, 6, 9, 8, $8,3,4,8$ e 10 . 


\subsubsection{NAMS}

Sem estrutura de correlação, o NAMS selecionou em média 15,12 variáveis, com a estrutura baixa de correlação selecionou em média 15,05 variáveis e com a estrutura de correlação alta 15,08 variáveis.

\subsubsection{RFS}

Sem estrutura de correlação, o método RFS selecionou em média, 15,07 variáveis, com a estrutura baixa de correlação, foram selecionadas 15,04 variáveis e com a estrutura alta de correlação 15,06 variáveis.

\subsubsection{LARS}

Sem estrutura de correlação, o LARS selecionou em média 18,72 variáveis, com a estrutura baixa de correlação foram selecionados 17,97 variáveis e com a estrutura alta de correlação, 17,85 variáveis. Também foi efetuada a seleção via LARS com número de coeficientes fixos.

\subsubsection{LASSO Bayesiano}

No caso sem correlação, o LASSO Bayesiano selecionou em média 15,01 variáveis, no caso com correlação baixa, em média 15,02 variáveis, e com a correlação alta, em média 15,05 variáveis.

\subsubsection{Spike-and-Slab LASSO}

Sem estrutura de correlação, o SSL selecionou em média 15,01 variáveis, e também 15,01 variáveis com a estrutura baixa de correlação. Com a estrutura alta de correlação, o método selecionou em média 15,05 variáveis.

\subsubsection{Comparação}

As Tabelas de comparação são as seguintes: Tabela 7 e Tabela 8:

\begin{tabular}{|c|c|c|c|c|c|c|c|c|c|c|c|c|c|c|c|c|c|c|c|c|c|}
\hline Variável & & 1 & 2 & 3 & 4 & 5 & 6 & 7 & 8 & 9 & 10 & 11 & 12 & 13 & 14 & 15 & 16 & 17 & 18 & 19 & 20 \\
\hline \multirow[t]{6}{*}{ Sem correlação } & NAMS & 100 & 1 & 100 & 100 & 100 & 100 & 100 & 100 & 100 & 2 & 3 & 3 & 3 & 100 & 100 & 100 & 100 & 100 & 100 & 100 \\
\hline & RFS & 100 & 1 & 100 & 100 & 100 & 100 & 100 & 100 & 100 & 1 & 1 & 1 & 3 & 100 & 100 & 100 & 100 & 100 & 100 & 100 \\
\hline & LARS & 100 & 73 & 100 & 100 & 100 & 100 & 100 & 100 & 100 & 68 & 72 & 81 & 78 & 100 & 100 & 100 & 100 & 100 & 100 & 100 \\
\hline & LARSF & 100 & 0 & 100 & 100 & 100 & 100 & 100 & 100 & 100 & 0 & 0 & 0 & 0 & 100 & 100 & 100 & 100 & 100 & 100 & 100 \\
\hline & BLASSO & 100 & 0 & 100 & 100 & 100 & 100 & 100 & 100 & 100 & 1 & 0 & 0 & 0 & 100 & 100 & 100 & 100 & 100 & 100 & 100 \\
\hline & SSL & 100 & 0 & 100 & 100 & 100 & 100 & 100 & 100 & 100 & 0 & 0 & 0 & 1 & 100 & 100 & 100 & 100 & 100 & 100 & 100 \\
\hline \multirow[t]{6}{*}{ Cor. baixa } & NAMS & 100 & 0 & 100 & 100 & 100 & 100 & 100 & 100 & 100 & 1 & 2 & 1 & 1 & 100 & 100 & 100 & 100 & 100 & 100 & 100 \\
\hline & RFS & 100 & 0 & 100 & 100 & 100 & 100 & 100 & 100 & 100 & 1 & 2 & 0 & 1 & 100 & 100 & 100 & 100 & 100 & 100 & 100 \\
\hline & LARS & 100 & 58 & 100 & 100 & 100 & 100 & 100 & 100 & 100 & 55 & 57 & 66 & 61 & 100 & 100 & 100 & 100 & 100 & 100 & 100 \\
\hline & LARSF & 100 & 0 & 100 & 100 & 100 & 100 & 100 & 100 & 100 & 0 & 0 & 0 & 0 & 100 & 100 & 100 & 100 & 100 & 100 & 100 \\
\hline & BLASSO & 100 & 0 & 100 & 100 & 100 & 100 & 100 & 100 & 100 & 0 & 1 & 0 & 1 & 100 & 100 & 100 & 100 & 100 & 100 & 100 \\
\hline & SSL & 100 & 0 & 100 & 100 & 100 & 100 & 100 & 100 & 100 & 1 & 0 & 0 & 0 & 100 & 100 & 100 & 100 & 100 & 100 & 100 \\
\hline \multirow[t]{6}{*}{ Cor. Alta } & NAMS & 100 & 2 & 100 & 100 & 100 & 100 & 100 & 100 & 100 & 1 & 0 & 3 & 2 & 100 & 100 & 100 & 100 & 100 & 100 & 100 \\
\hline & RFS & 100 & 0 & 100 & 100 & 100 & 100 & 100 & 100 & 100 & 1 & 0 & 3 & 2 & 100 & 100 & 100 & 100 & 100 & 100 & 100 \\
\hline & LARS & 100 & 100 & 100 & 100 & 100 & 100 & 100 & 100 & 100 & 49 & 48 & 46 & 42 & 100 & 100 & 100 & 100 & 100 & 100 & 100 \\
\hline & LARSF & 1 & 99 & 100 & 100 & 100 & 100 & 100 & 100 & 100 & 0 & 0 & 0 & 0 & 100 & 100 & 100 & 100 & 100 & 100 & 100 \\
\hline & BLASSO & 100 & 2 & 100 & 100 & 100 & 100 & 100 & 100 & 100 & 0 & 1 & 1 & 1 & 100 & 100 & 100 & 100 & 100 & 100 & 100 \\
\hline & SSL & 100 & 0 & 100 & 100 & 100 & 100 & 100 & 100 & 100 & 1 & 0 & 3 & 1 & 100 & 100 & 100 & 100 & 100 & 100 & 100 \\
\hline
\end{tabular}

Tabela $7-15$ variáveis explicativas 
Nota-se que, novamente, todos os métodos, com exceção do "LARS - tamanho fixo", todos os métodos selecionaram todas as variáveis corretas todas as vezes.

\begin{tabular}{cccccc}
\hline Caso & Método & \# médio de var. sel. & d. p. \# & $\widehat{E Q M}$ & $\%$ correto \\
\hline não correlacionadas & NAMS & 15,12 & 0,4980771 & 0,19533991 & 92 \\
& RFS & 15,07 & 0,2564324 & 0,19335823 & 93 \\
& LARS & 18,72 & 1,1288682 & 0,26935244 & 0 \\
& LARS - TF & 15,00 & 0 & 2,10689347 & 100 \\
& LASSO B. & 15,04 & 0,1969464 & 0,18846273 & 96 \\
& SSL & 15,01 & 0,1 & 0,18950211 & 99 \\
\hline Correlação Baixa & NAMS & 15,05 & 0,2190429 & 0,21790310 & 95 \\
& RFS & 15,04 & 0,1969464 & 0,21602454 & 96 \\
& LARS & 17,97 & 1,2589478 & 0,30337551 & 4 \\
& LARS - TF & 15,00 & 0 & 1,65667304 & 100 \\
& LASSO B. & 15,02 & 0,1407053 & 0,21804349 & 98 \\
& SSL & 15,01 & 0,1 & 0,21918034 & 99 \\
\hline Correlação Alta & NAMS & 15,08 & 0,3074824 & 0,44680589 & 93 \\
& RFS & 15,06 & 0,2777980 & 0,44110028 & 95 \\
& LARS & 17,85 & 1,1225422 & 0,58671907 & 0 \\
& LARS - TF & 15,00 & 0 & 7,68462066 & 1 \\
& LASSO B. & 15,06 & 0,2777980 & 0,43544430 & 95 \\
& SSL & 15,05 & 0,2611165 & 0,43732571 & 96 \\
\hline
\end{tabular}

Tabela 8 - Comparação dos métodos - 15 variáveis

No caso de 15 variáveis explicativas, o LASSO Bayesiano e o SSL selecionaram os menores modelos (no caso de variáveis altamente correlacionadas, RFS também selecionou em média, modelos com o mesmo tamanho do LASSO bayesiano). No quesito do menor Erro Quadrático Médio, houve uma variação: No caso de variáveis sem estrutura de correlação, o LASSO Bayesiano foi o melhor, seguido pelo SSL. Com variáveis pouco correlacionadas, a RFS teve o menor $\widehat{E Q M}$, seguido pelo NAMS, e no caso de variáveis altamente correlacionadas, novamente o LASSO Bayesiano teve o melhor resultado, seguido pelo SSL. No percentual de acertos, novamente, entre os modelos aplicáveis em situação prática, o SSL foi o melhor em todos casos.

Na construção teórica do LARS com o número de preditores fixos, observa-se o mesmo resultado do caso anterior: No caso sem estrutura de correlação, bem como com a estrutura baixa, seu percentual de acertos é muito alto, sendo inclusive o melhor entre todos os métodos. No caso de variáveis altamente correlacionadas porém, só houve um acerto. Isso foi devido ao alto percentual de seleção da variável 2, cujo coeficiente real era zero.

Nota-se que os resultados do NAMS, RFS, LASSO Bayesiano e SSL foram similares.

\subsection{Conclusão}

Após analisar todos os casos, constatou-se que, na simulação estudada, todos os métodos selecionaram para o modelo final todas as variáveis explicativas cujos coeficientes eram diferentes 
de zero, todas as vezes (apenas a situação teórica do LARS com o número de coeficientes fixos não teve esse resultado, o que era esperado, pois sendo o número de coeficientes fixado qualquer seleção errada já leva necessariamente ao descarte de uma variável correta do modelo).

Assim, as diferenças encontradas foram por conta do tamanho dos modelos (que no caso equivale a quantas variáveis explicativas desnecessárias foram incluídas nos modelos finais) e da precisão da estimação, medida pelo $\widehat{E Q M}$ do estimador.

Nesses termos, o LARS teve um resultado inferior, selecionando muitas variáveis desnecessárias. Surpreendentemente, o NAMS mostrou muitos resultados iguais ou superiores à RFS, apesar desta última ter sido desenvolvida por dois dos mesmos autores do primeiro e publicada posteriormente.

Os métodos Bayesianos estudados, o LASSO Bayesiano (às vezes chamado na literatura de BLASSO) e o Sike-and-Slab LASSO apresentaram os melhores resultados, com o SSL sendo melhor na maioria dos casos. A diferença entre os dois, bem como a diferença entre eles e os métodos com base em pseudovariáveis, foi pequena.

A diferença entre os métodos no quesito de tempo computacional, por outro lado, é bastante grande.

O NAMS requer a maior capacidade computacional, já que necessita da simulação de 4000 vetores de variáveis explicadas acrescidas de perturbação. A RFS também requer uma grande capacidade computacional para criar os vetores de pseudovariáveis explicativas. Em seguida vem o LASSO Bayesiano, que utiliza o amostrador de Gibbs para o cálculo dos estimadores. Os mais velozes são o SSL e o LARS, que não dependem de nenhum tipo de simulação. Entre eles, o LARS é o mais veloz, já que tem um número pequeno de etapas, igual ao número de variáveis explicativas.

Assim sendo, levando em conta todos os aspectos, os métodos Bayesianos se saíram melhor nos resultados da simulação.

Uma possível explicação para esse fato de todos os métodos selecionarem todas as variáveis todas as vezes é que, na simulação feita, o tamanho dos $\beta^{\prime} s$ era grande em relação ao desvio padrão, o que diminui a possibilidade de os $\beta^{\prime} s$ verdadeiros serem erroneamente considerados nulos. Assim, métodos que selecionam modelos menores, como o SSL, têm grande vantagem.

Caso a simulação fosse feita com coeficientes mais baixos (digamos, de 0,5 a 2,5) e mantida a variância, é esperado que todos os métodos tenham sua seleção piorada e seria necessário estudar qual modelo seria o melhor nessa situação. Resultados preliminares indicam que a eficiência do SSL, que tem uma tendência a selecionar modelos enxutos, tem uma piora significativa e o LASSO, por selecionar mais variáveis que o necessário, tende a selecionar as variáveis corretas mais vezes, tornando-o mais competitivo. Além disso, os resultados do BLASSO também parecem ser muito positivos.

Além disso, existem outras possibilidades interessantes de estudos de simulação a serem executados no futuro, como no caso de dados superdimensionados, isto é, nos quais existem mais variáveis explicativas do que observações (geralmente na casa das centenas) e também de 
Big Data, caso no qual o número de observações é altíssimo, chegando a milhões, e o tempo computacional passa a ser de extrema importância.

Os artigos analisados neste trabalho representam algumas dentre muitas possibilidades de pesquisa no campo de seleção de variáveis no contexto de regressão linear. Os estimadores com base na inclusão de pseudovariáveis mostraram resultados muito bons nos estudos de simulação e nas bases de dados reais, e caso algoritmos mais rápidos e eficientes sejam desenvolvidos, tais métodos seriam uma grande adição às técnicas tradicionais de seleção de variáveis. $\mathrm{O}$ procedimento LARS é novo conceito para a estimação de parâmetros do modelo, extremamente eficiente do ponto de vista computacional e que permite obter todas as soluções do LASSO tradicional.

Finalmente, os métodos bayesianos se mostram ainda mais promissores, oferecendo algoritmos eficientes computacionalmente e com excelentes resultados de estimação.

\section{.1 Apêndice A}

No espaço $\mathcal{L}(\boldsymbol{X})$ o cosseno entre dois vetores $x$ e $y$ quaisquer é dado por:

$$
\cos \theta=\frac{x^{\prime} y}{L_{x} L_{y}}=\frac{x^{\prime} y}{\sqrt{x^{\prime} x} \sqrt{y^{\prime} y}}
$$

Em nosso caso temos:

$$
u_{\mathcal{A}}=X_{\mathcal{A}} w_{\mathcal{A}}=X_{\mathcal{A}} A_{\mathcal{A}} G_{\mathcal{A}}^{-1} 1_{\mathcal{A}}=\frac{X_{\mathcal{A}} G_{\mathcal{A}}^{-1} 1_{\mathcal{A}}}{\left(1_{\mathcal{A}}^{\prime} G_{\mathcal{A}}^{-1} 1_{\mathcal{A}}\right)^{/ \frac{1}{2}}} .
$$

Utilizando a fórmula (1) para calcular o cosseno do ângulo entre $\boldsymbol{u}_{\mathcal{A}}$ e a primeira linha da matriz $X_{\mathcal{A}}, s_{1} \boldsymbol{x}_{1}$ obtém-se:

$$
\cos \left(\theta_{1}\right)=\frac{\boldsymbol{u}_{\mathcal{A}} s_{1} \boldsymbol{x}_{\mathbf{1}}}{\sqrt{\boldsymbol{u}_{\mathcal{A}}^{\prime} \boldsymbol{u}_{\mathcal{A}}} \sqrt{\boldsymbol{x}_{\mathbf{1}}^{\prime} \boldsymbol{x}_{\mathbf{1}}}}=s_{1} \boldsymbol{x}_{\mathbf{1}}^{\prime} \boldsymbol{u}_{\mathcal{A}}=\boldsymbol{u}_{\mathcal{A}}^{\prime} s_{1} \boldsymbol{x}_{\mathbf{1}},
$$

Isso porque, $L_{s 1 \boldsymbol{x}_{\mathbf{1}}}=\sum_{i=1}^{n} x_{i, 1}^{2}=1$, pois as variáveis foram centralizadas. $L_{\boldsymbol{u}_{\mathcal{A}}}$ também é igual a 1, pois:

$$
\begin{gathered}
\boldsymbol{u}_{\mathcal{A}}^{\prime} \boldsymbol{u}_{\mathcal{A}}=\boldsymbol{w}_{\mathcal{A}}^{\prime} X_{\mathcal{A}}^{\prime} \boldsymbol{w}_{\mathcal{A}} X_{\mathcal{A}}=\mathbf{1}_{\mathcal{A}}^{\prime} G_{\mathcal{A}}^{\prime-1} A_{\mathcal{A}}^{\prime} X_{\mathcal{A}}^{\prime} X_{\mathcal{A}} A_{\mathcal{A}} G_{\mathcal{A}}^{-1} \mathbf{1}_{\mathcal{A}}= \\
\mathbf{1}_{\mathcal{A}}^{\prime} G_{\mathcal{A}}^{\prime-1} A_{\mathcal{A}}^{\prime} A_{\mathcal{A}} \mathbf{1}_{\mathcal{A}}= \\
\mathbf{1}_{\mathcal{A}}^{\prime} G_{\mathcal{A}}^{\prime-1} \mathbf{1}_{\mathcal{A}} A_{\mathcal{A}}^{\prime} A_{\mathcal{A}}= \\
A^{-2} A^{2}=1
\end{gathered}
$$

Perceba-se além disso, que o resultado $s_{1} \boldsymbol{x}_{\mathbf{1}}^{\prime} \boldsymbol{u}_{\mathcal{A}}$ equivale exatamente a multiplicar a primeira linha da matriz $\boldsymbol{X}_{\mathcal{A}}^{\prime}$ pelo vetor $\boldsymbol{u}_{\mathcal{A}}$. 
Com isso, podemos também obter o resultado tomando a primeira linha do vetor: $A_{\mathcal{A}} \mathbf{1}_{\mathcal{A}}=\left(\mathbf{1}_{\mathcal{A}}^{\prime} G_{\mathcal{A}}^{-1} \mathbf{1}_{\mathcal{A}}\right)^{\frac{1}{2}}$, pois:

$$
\begin{gathered}
X_{\mathcal{A}}^{\prime} \boldsymbol{u}_{\mathcal{A}}=X_{\mathcal{A}}^{\prime} X_{\mathcal{A}} A_{\mathcal{A}} G_{A}^{-1} \mathbf{1}_{\mathcal{A}}= \\
X_{\mathcal{A}}^{\prime} X_{\mathcal{A}}\left(X_{\mathcal{A}}^{\prime} X_{\mathcal{A}}\right)^{-1} A_{\mathcal{A}} \mathbf{1}_{\mathcal{A}}= \\
A_{\mathcal{A}} \mathbf{1}_{\mathcal{A}}=\left(\mathbf{1}_{\mathcal{A}}^{\prime} G_{\mathcal{A}}^{-1} \mathbf{1}_{\mathcal{A}}\right)^{\frac{1}{2}} \mathbf{1}_{\mathcal{A}}
\end{gathered}
$$

Assim, o cosseno do ângulo (primeira linha do vetor) é $\left(\mathbf{1}_{\mathcal{A}}\right)^{\frac{1}{2}}$. Ao calcular o cosseno da segunda linha de $X_{\mathcal{A}}$ com o vetor $\boldsymbol{u}_{\mathcal{A}}$ obtemos o resultado:

$$
\cos \left(\theta_{2}\right)=\frac{\boldsymbol{u}_{\mathcal{A}}^{\prime} s_{2} \boldsymbol{x}_{\mathbf{2}}}{1 \times 1}=s_{2} \boldsymbol{x}_{\mathbf{2}}^{\prime} \boldsymbol{u}_{\mathcal{A}}
$$

Este resultado corresponde à segunda linha de $X_{\mathcal{A}}^{\prime} \boldsymbol{u}_{\mathcal{A}}$, que também é igual à segunda linha de $A_{\mathcal{A}} \mathbf{1}_{\mathcal{A}},\left(\left(\mathbf{1}_{\mathcal{A}}\right)^{\frac{1}{2}}\right)$. Portanto $\cos \theta_{1}=\cos \theta_{2}$, e como $\theta_{1}$ e $\theta_{2}$ estão entre 0 e $90^{\circ}, \theta_{1}=\theta_{2}$.

Mais que isso, como a multiplicação da matriz inteira $X_{\mathcal{A}}$ pelo vetor $\boldsymbol{u}_{\mathcal{A}}$ é $\boldsymbol{A}_{\mathcal{A}}$ (um escalar) multiplicado por um vetor de 1 's, o resultado é um vetor com $\mathcal{A}$ valores $\left(\left(\mathbf{1}_{\mathcal{A}}\right)^{\frac{1}{2}}\right)$. Como cada linha desse vetor representa a multiplicação de uma linha da matriz $X_{\mathcal{A}}^{\prime}$ com o vetor $\boldsymbol{u}_{\mathcal{A}}$, que por sua vez representa o cosseno do ângulo entre as linhas da matriz $X_{\mathcal{A}}$ e $\boldsymbol{u}_{\mathcal{A}}$, e todas as linhas deste vetor são iguais, termina-se a demonstração de que o vetor $\boldsymbol{u}_{\mathcal{A}}$ forma ângulos iguais com cada coluna da matriz $X_{\mathcal{A}}$

\section{.2 Apêndice B - funções para a RFS e ajustes para o NAMS}

Os seguintes ajustes foram feitos no programa dos autores para o NAMS:

1. Substituir linha 36 por: $y m<-\operatorname{matrix}(\operatorname{rep}(\mathrm{y}, \mathrm{B}), \mathrm{nrow}=\mathrm{B}$, byrow $=$ TRUE $)$

2. Substituir linha 42 por: for (i in 1:4)av[i,]<-colMeans(as.data.frame(mse.a[,,i] ))

3. Substituir linha 57 por: cat(,fill=TRUE)

4. Substituir linha 58 por: cat("Variables Selected =",colnames(x),fill=TRUE)

Os ajustes foram os seguintes: A função mean não mais retorna a média das colunas de um data-frame, ao invés disso, utiliza-se a função colMeans. Além disso, o uso de "T"e "F"no lugar de "TRUE" e "FALSE" pode geral problemas caso sejam utilizados dentro de funções. No computador utilizado para fazer as simulações, por exemplo, o uso de " $\mathrm{T}$ "retornava a mensagem de operador inválido.

Também sugere-se as seguintes modificações:

- Substituir linha 55 por: if $(\operatorname{size} 1==1)\{\bmod <-\operatorname{lm}(y 1)\}$ else $\{\bmod <-\operatorname{lm}(y \mathrm{x}+0)\}$

- Substituir linha 1 por: nams<-function $(\mathrm{x}, \mathrm{y}, \mathrm{B}=4000)\{$ 
- Eliminar linha 8

- Substituir linha 56 (55 caso tenha sido realizada a alteração anterior) por: print(res<data.frame(m,n,B,tau,ng,alphahat))

Essas alterações passam a fazer o NAMS estimar modelos sem intercepto (para modelos cujas variáveis foram centralizadas, como é o caso deste trabalho) e eliminam a necessidade de utilizar uma seed fixa na estimação (algo que não é proposto no artigo).

Segue agora a função desenvolvida para a implementação do método RFS no R, bem como um algoritmo para o método de seleção forward, que é utilizado em trechos do código. Esse algoritmo foi desenvolvido para seguir passo a passo e de maneira literal todo o processo apresentado no artigo. Por conta disso, é possível que existam implementações computacionais mais eficientes.

\section{.2 .1 Forward}

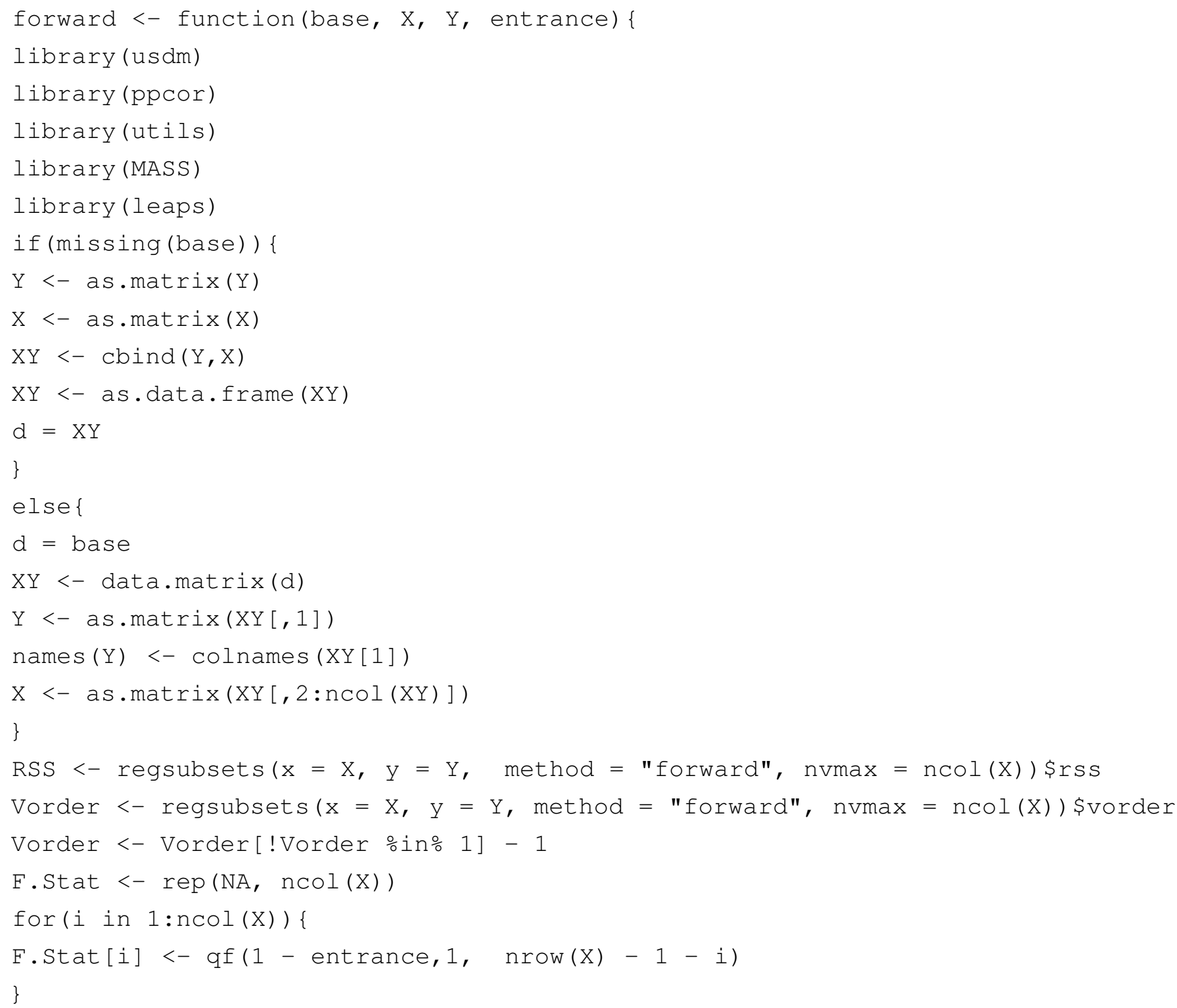




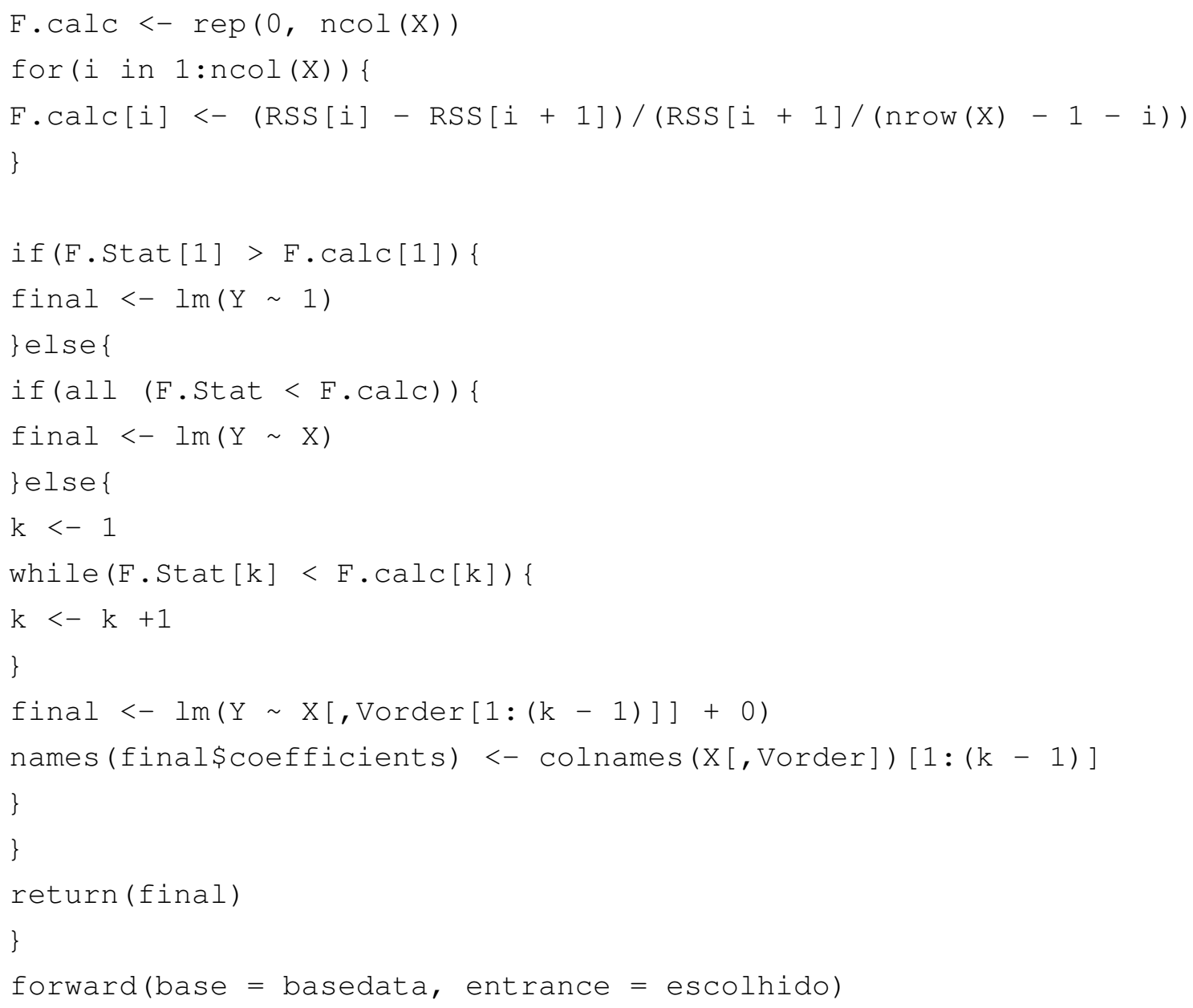

\section{.2 .2 RFS}

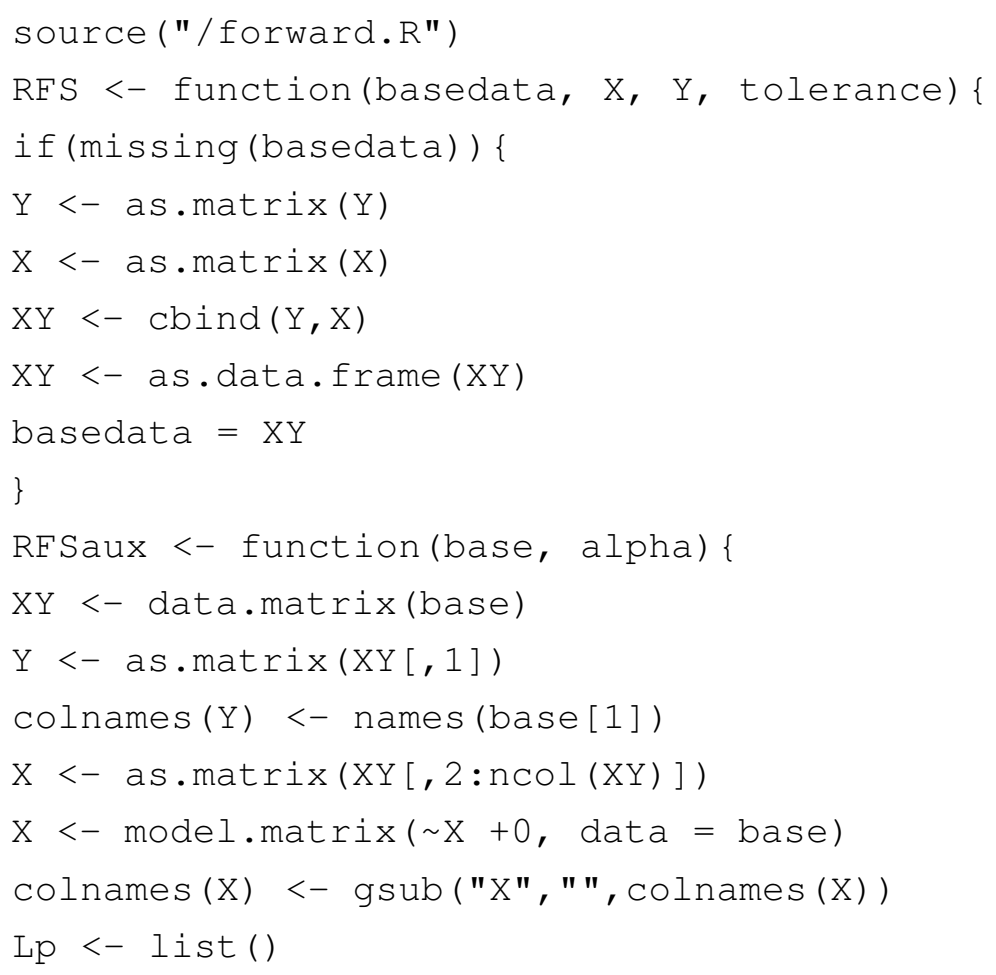




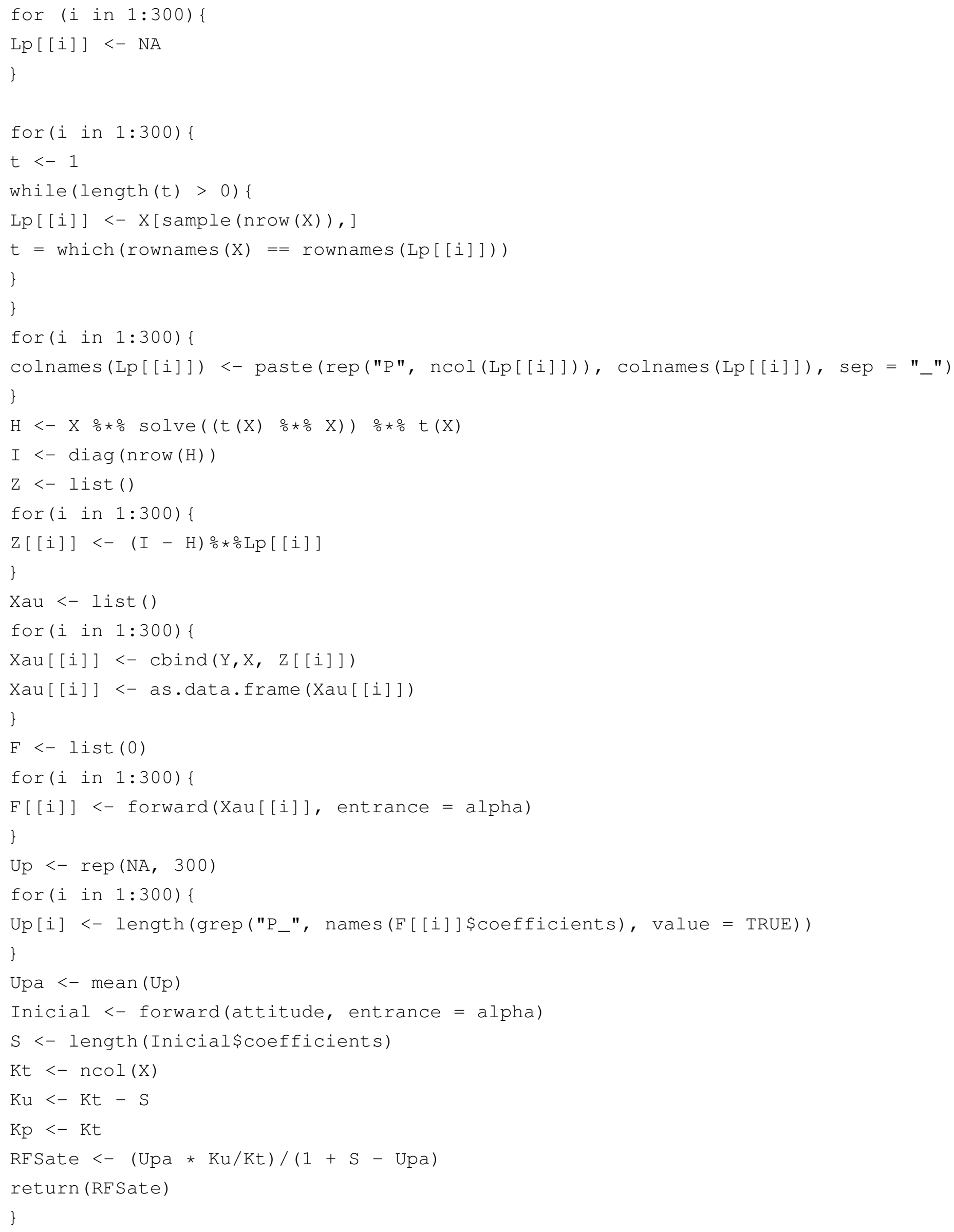




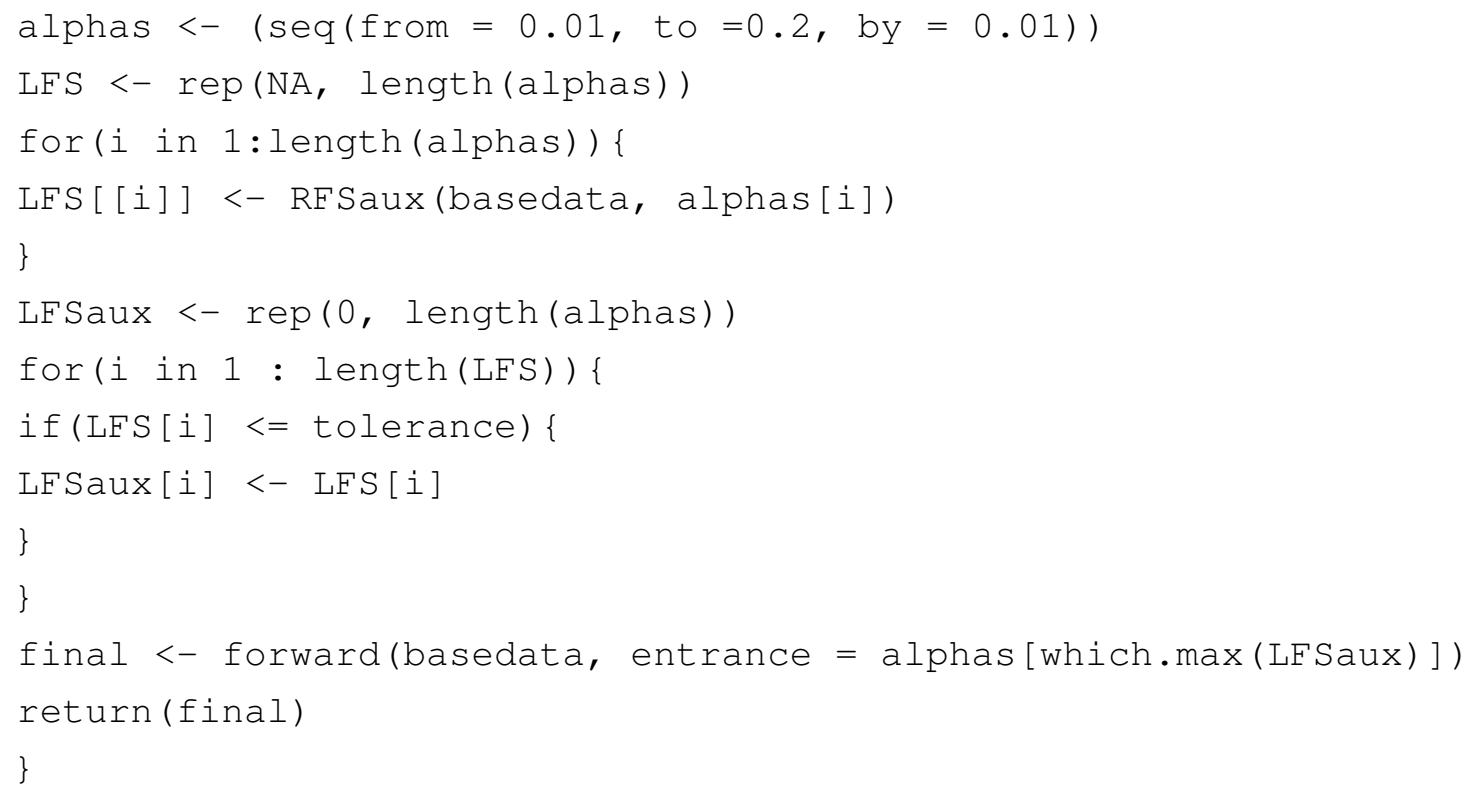





\section{Referências}

BREIMAN, L.; SPECTOR, P. Submodel selection and evaluation in regression. the x-random case. International statistical review/revue internationale de Statistique, JSTOR, p. 291-319, 1992. Citado na página 6 .

CASELLA, G.; GEORGE, E. I. Explaining the gibbs sampler. The American Statistician, Taylor \& Francis, v. 46, n. 3, p. 167-174, 1992. Citado 2 vezes nas páginas 38 e 39.

CHATTERJEE, S.; HADI, A.; PRICE, B. Regression Analysis by Example. [S.l.]: Wiley, 1999. (Wiley Series in Probability and Statistics). ISBN 9780471319467. Citado 2 vezes nas páginas 53 e 54.

CHHIKARA, R.; FOLKS, J. L. The Inverse Gaussian Distribution: Theory: Methodology, and Applications. [S.l.]: CRC Press, 1988. v. 95. Citado na página 41.

COOK, J. R.; STEFANSKI, L. A. Simulation-extrapolation estimation in parametric measurement error models. Journal of the American Statistical association, Taylor \& Francis Group, v. 89, n. 428, p. 1314-1328, 1994. Citado na página 18.

EFRON, B. et al. Least angle regression. The Annals of statistics, Institute of Mathematical Statistics, v. 32, n. 2, p. 407-499, 2004. Citado 5 vezes nas páginas 3, 7, 9, 13 e 54 .

ERDÉLYI, A. Asymptotic expansions of fourier integrals involving logarithmic singularities. Journal of the Society for Industrial and Applied Mathematics, SIAM, v. 4, n. 1, p. 38-47, 1956. Citado na página 32.

GEORGE, E. I.; FOSTER, D. P. Calibration and empirical bayes variable selection. Biometrika, JSTOR, p. 731-747, 2000. Citado 2 vezes nas páginas 33 e 34.

JOHNSON, R. A.; WICHERN, D. W. Applied multivariate statistical analysis. 6. ed. [S.l.]: Prentice hall Upper Saddle River, NJ, 2007. 800 p. Citado na página 14.

LI, F.; ZHANG, N. R. Bayesian variable selection in structured high-dimensional covariate spaces with applications in genomics. Journal of the American statistical association, Taylor \& Francis, v. 105, n. 491, p. 1202-1214, 2010. Citado 2 vezes nas páginas 49 e 51.

LIMA, E. L. Algebra Linear. 5. ed. [S.1.]: IMPA, Rio de Janeiro, 2001. 357 pp. p. Citado 3 vezes nas páginas 13, 14 e 15.

LUO, X.; STEFANSKI, L. A.; BOOS, D. D. Tuning variable selection procedures by adding noise. Technometrics, Taylor \& Francis, v. 48, n. 2, p. 165-175, 2006. Citado 6 vezes nas páginas $3,4,17,20,27$ e 55.

MALLOWS, C. L. Some comments on c p. Technometrics, Taylor \& Francis Group, v. 15, n. 4, p. 661-675, 1973. Citado 2 vezes nas páginas 3 e 13.

MALLOWS, C. L. More comments on cp. Technometrics, Taylor \& Francis Group, v. 37, n. 4, p. 362-372, 1995. Citado 2 vezes nas páginas 3 e 21.

PARK, T.; CASELLA, G. The bayesian lasso. Journal of the American Statistical Association, Taylor \& Francis, v. 103, n. 482, p. 681-686, 2008. Citado 5 vezes nas páginas 3, 4, 34, 49 e 53. 
PARK, T.; CASELlA, G. The bayesian lasso. 2008. Disponível em: < https://pdfs. semanticscholar.org/5b51/ce3bbe7791e1533be7d4d76b2452bf043954.pdf>. Citado 3 vezes nas páginas 34, 41 e 43.

ROBERT, C. P.; CASELlA, G. Monte carlo optimization. In: Monte Carlo Statistical Methods. [S.1.]: Springer, 2004. p. 157-204. Citado na página 42.

ROČKOVÁ, V.; GEORGE, E. I. The spike-and-slab lasso. Journal of the American Statistical Association, Taylor \& Francis, n. just-accepted, 2016. Citado na página 3.

ROČKOVÁ, V.; GEORGE, E. I. The spike-and-slab lasso. Journal of the American Statistical Association, Taylor \& Francis, n. just-accepted, 2016. Citado 6 vezes nas páginas 4, 44, 46, 47, 48 e 49.

RYAN, T. P. Modern regression methods. [S.1.]: John Wiley \& Sons, 2008. v. 655. Citado 2 vezes nas páginas 3 e 4 .

TIBSHIRANI, R. Regression shrinkage and selection via the lasso. Journal of the Royal Statistical Society. Series B (Methodological), JSTOR, p. 267-288, 1996. Citado 3 vezes nas páginas 5, 6 e 29 .

WU, Y.; BOOS, D. D.; STEFANSKI, L. A. Controlling variable selection by the addition of pseudovariables. Journal of the American Statistical Association, v. 102, n. 477, p. 235-243, 2007. Disponível em: <http://dx.doi.org/10.1198/016214506000000843>. Citado 5 vezes nas páginas $4,17,21,26$ e 27.

YUAN, M.; JOSEPH, V. R.; LIN, Y. An efficient variable selection approach for analyzing designed experiments. Technometrics, Taylor \& Francis, v. 49, n. 4, p. 430-439, 2007. Citado 3 vezes nas páginas 4,31 e 33 .

YUAN, M.; LIN, Y. Efficient empirical bayes variable selection and estimation in linear models. Journal of the American Statistical Association, Taylor \& Francis, v. 100, n. 472, p. 1215-1225, 2005. Citado 5 vezes nas páginas 3, 29, 32, 45 e 49 . 